\title{
Exploring Cosmic Origins with CORE: Survey requirements and mission design
}

J. Delabrouille, ${ }^{1}$ P. de Bernardis, ${ }^{2}$ F. R. Bouchet, ${ }^{3}$ A. Achúcarro,

P. A. R. Ade, R. Allison, F. Arroja, E. Artal, M. Ashdown,

C. Baccigalupi, M. Ballardini, A. J. Banday, R. Banerji,

D. Barbosa, J. Bartlett, N. Bartolo, S. Basak, J. J. A. Baselmans,

K. Basu, E. S. Battistelli, R. Battye, D. Baumann, A. Benoît,

M. Bersanelli, A. Bideaud, M. Biesiada, M. Bilicki, A. Bonaldi,

M. Bonato, J. Borrill, F. Boulanger, T. Brinckmann, M. L. Brown,

M. Bucher, C. Burigana, A. Buzzelli, G. Cabass, Z.-Y. Cai,

M. Calvo, A. Caputo, C.-S. Carvalho, F. J. Casas, G. Castellano,

A. Catalano, A. Challinor, I. Charles, J. Chluba, D. L. Clements,

S. Clesse, S. Colafrancesco, I. Colantoni, D. Contreras,

A. Coppolecchia, M. Crook, G. D'Alessandro, G. D'Amico,

A. da Silva, M. de Avillez, G. de Gasperis, M. De Petris,

G. de Zotti, L. Danese, F.-X. Désert, V. Desjacques,

E. Di Valentino, C. Dickinson, J. M. Diego, S. Doyle, R. Durrer,

C. Dvorkin, H.-K. Eriksen, J. Errard, S. Feeney,

R. Fernández-Cobos, F. Finelli, F. Forastieri, C. Franceschet,

U. Fuskeland, S. Galli, R. T. Génova-Santos, M. Gerbino,

E. Giusarma, A. Gomez, J. González-Nuevo, S. Grandis,

J. Greenslade, J. Goupy, S. Hagstotz, S. Hanany, W. Handley,

S. Henrot-Versillé, C. Hernández-Monteagudo,

C. Hervias-Caimapo, M. Hills, M. Hindmarsh, E. Hivon,

D. T. Hoang, D. C. Hooper, B. Hu, E. Keihänen, R. Keskitalo,

K. Kiiveri, T. Kisner, T. Kitching, M. Kunz, H. Kurki-Suonio,

G. Lagache, L. Lamagna, A. Lapi, A. Lasenby, M. Lattanzi,

A. M. C. Le Brun, J. Lesgourgues, M. Liguori, V. Lindholm,

J. Lizarraga, G. Luzzi, J. F. Macias-Pérez, B. Maffei,

N. Mandolesi, S. Martin, E. Martinez-Gonzalez, C.J.A.P. Martins, 
S. Masi, M. Massardi, S. Matarrese, P. Mazzotta, D. McCarthy,

A. Melchiorri, J.-B. Melin, A. Mennella, J. Mohr, D. Molinari,

A. Monfardini, L. Montier, P. Natoli, M. Negrello, A. Notari,

F. Noviello, F. Oppizzi, C. O'Sullivan, L. Pagano, A. Paiella,

E. Pajer, D. Paoletti, S. Paradiso, R. B. Partridge, G. Patanchon,

S. P. Patil, O. Perdereau, F. Piacentini, M. Piat, G. Pisano,

L. Polastri, G. Polenta, A. Pollo, N. Ponthieu, V. Poulin, D. Prêle,

M. Quartin, A. Ravenni, M. Remazeilles, A. Renzi, C. Ringeval,

D. Roest, M. Roman, B. F. Roukema, J.-A. Rubiño-Martin,

L. Salvati, D. Scott, S. Serjeant, G. Signorelli, A. A. Starobinsky,

R. Sunyaev, C. Y. Tan, A. Tartari, G. Tasinato, L. Toffolatti,

M. Tomasi, J. Torrado, D. Tramonte, N. Trappe, S. Triqueneaux,

M. Tristram, T. Trombetti, M. Tucci, C. Tucker, J. Urrestilla,

J. Väliviita, R. Van de Weygaert, B. Van Tent, V. Vennin,

L. Verde, G. Vermeulen, P. Vielva, N. Vittorio, F. Voisin,

C. Wallis, B. Wandelt, I. Wehus, J. Weller, K. Young,

M. Zannoni, for the CORE collaboration

${ }^{1}$ APC, Astroparticule et Cosmologie, Université Paris Diderot, CNRS/IN2P3, CEA/lrfu, Observatoire de Paris Sorbonne Paris Cité, 10, rue Alice Domon et Léonie Duquet, 75205

Paris Cedex 13, France

${ }^{2}$ Physics Department, Sapienza University of Rome and INFN Sezione di Roma, Piazzale Aldo Moro 2, 00185, Rome, Italy

${ }^{3}$ Institut d'Astrophysique de Paris, (UMR 7095: CNRS \& UPMC Sorbonne Universités), F-75014, Paris, France

E-mail: delabrouille@apc.in2p3.fr

\section{Abstract.}

Future observations of cosmic microwave background (CMB) polarisation have the potential to answer some of the most fundamental questions of modern physics and cosmology, including: What physical process gave birth to the Universe we see today? What are the dark matter and dark energy that seem to constitute $95 \%$ of the energy density of the Universe? Do we need extensions to the standard model of particle physics and fundamental interactions? Is the $\Lambda \mathrm{CDM}$ cosmological scenario correct, or are we missing an essential piece of the puzzle? In this paper, we list the requirements for a future CMB polarisation survey addressing these scientific objectives, and discuss the design drivers of the CORE space mission proposed to ESA in answer to the "M5" call for a medium-sized mission. The rationale and options, and the methodologies used to assess the mission's performance, are of interest to other future CMB mission design studies. CORE has 19 frequency channels, distributed over a broad frequency range, spanning the $60-600 \mathrm{GHz}$ interval, to control astrophysical foreground emission. The angular resolution ranges from $2^{\prime}$ to $18^{\prime}$, and the aggregate CMB sensitivity is about $2 \mu \mathrm{K}$.arcmin. The observations are made with a single integrated focal-plane instrument, consisting of an array of 2100 cryogenically-cooled, linearly-polarised detectors at the focus of a 1.2-m aperture cross-Dragone telescope. The mission is designed 
to minimise all sources of systematic effects, which must be controlled so that no more than $10^{-4}$ of the intensity leaks into polarisation maps, and no more than about $1 \%$ of $E$-type polarisation leaks into $B$-type modes. CORE observes the sky from a large Lissajous orbit around the Sun-Earth L2 point on an orbit that offers stable observing conditions and avoids contamination from sidelobe pick-up of stray radiation originating from the Sun, Earth, and Moon. The entire sky is observed repeatedly during four years of continuous scanning, with a combination of three rotations of the spacecraft over different timescales. With about $50 \%$ of the sky covered every few days, this scan strategy provides the mitigation of systematic effects and the internal redundancy that are needed to convincingly extract the primordial $B$-mode signal on large angular scales, and check with adequate sensitivity the consistency of the observations in several independent data subsets. CORE is designed as a "near-ultimate" CMB polarisation mission which, for optimal complementarity with ground-based observations, will perform the observations that are known to be essential to CMB polarisation science and cannot be obtained by any other means than a dedicated space mission. It will provide well-characterised, highly-redundant multi-frequency observations of polarisation at all the scales where foreground emission and cosmic variance dominate the final uncertainty for obtaining precision CMB science, as well as $2^{\prime}$ angular resolution maps of high-frequency foreground emission in the $300-600 \mathrm{GHz}$ frequency range, essential for complementarity with future ground-based observations with large telescopes that can observe the CMB with the same beamsize. 


\section{Contents}

1 Introduction $\quad 1$

2 Overview of CORE $\quad 3$

3 Scientific objectives $\quad 7$

3.1 Inflation 8

3.2 The cosmological model $\quad 9$

3.3 Fundamental particles and interactions 11

$\begin{array}{lll}3.4 & \text { Structures } & 11\end{array}$

$\begin{array}{lll}3.5 & \text { Legacy } & 13\end{array}$

4 Survey requirements $\quad \mathbf{1 5}$

4.1 The need for a space mission $\quad 15$

$\begin{array}{lll}\text { 4.1.1 Atmosphere } & 17\end{array}$

$\begin{array}{lll}\text { 4.1.2 } & \text { Astrophysical foregrounds } & 17\end{array}$

$\begin{array}{lll}4.1 .3 & \text { Systematic effects } & 19\end{array}$

$\begin{array}{ll}\text { 4.1.4 Why space - summary } & 19\end{array}$

$\begin{array}{ll}4.2 & \text { What survey? } \\ & 20\end{array}$

4.3 Sky coverage 21

4.4 Sensitivity and angular resolution 21

4.5 Frequency channels 25

4.6 Systematic effects 27

4.7 Flexibility, safety margins, and redundancy 28

$\begin{array}{lll}4.8 & \text { Survey requirements and goals - summary } & 29\end{array}$

5 Mission design $\quad 30$

$\begin{array}{lll}5.1 & \text { Practical constraints } & 30\end{array}$

$\begin{array}{lll}5.2 & \text { Orbit } & 30\end{array}$

5.3 Observing strategy 31

5.4 Mission phases and operations 34

$\begin{array}{lll}5.5 & \text { Telemetry } & 35\end{array}$

6 Payload $\quad 35$

6.1 Instrument design 37

$\begin{array}{lll}6.2 & \text { Telescope } & 37\end{array}$

6.3 Shielding against sidelobe stray light 38

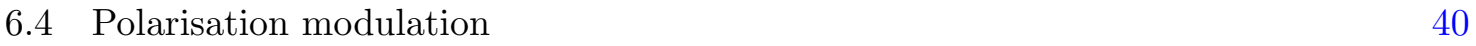

6.4.1 Technical complexity 40

6.4.2 Impact on science performance 41

6.4.3 Mitigation or generation of systematics? 42

6.5 Cooling chain 43

6.6 Mass and power budgets 44

6.7 Scanning strategy and payload design 44 
7 Controlling systematic effects

7.1 Systematic-correction mapmaking 45

$\begin{array}{lll}7.2 & \text { Bandpass leakage correction } & 47\end{array}$

8 Options $\quad 48$

8.1 Descoping options 48

8.2 Upgrades 50

9 Discussion $\quad \mathbf{5 1}$

9.1 Complementarity with sub-orbital experiments 52

10 Conclusion $\quad 53$

A Impact of atmosphere on ground-based CMB observations $\quad 56$

$\begin{array}{lll}\text { A.1 Atmosphere and detector sensitivity } & 56\end{array}$

$\begin{array}{lll}\text { A.2 Required observing time and focal-plane area } & 57\end{array}$

$\begin{array}{lll}\text { A.3 Atmospheric emission fluctuations } & 58\end{array}$

B Scan strategy optimisation $\quad \mathbf{5 9}$

$\begin{array}{lll}\text { B.1 Main requirements and design drivers } & 59\end{array}$

$\begin{array}{lll}\text { B.2 Practical constraints } & 59\end{array}$

$\begin{array}{lll}\text { B.3 Sampling } & 61\end{array}$

B.4 Optimisation $\quad 63$

\section{Introduction}

In the past few decades, the field of cosmology has undergone a period of dramatically rapid progress in which a standard model of cosmology has emerged, $\Lambda$ CDM. The precision with which this model has been constrained has been largely driven by studies of the anisotropies in the cosmic microwave background (CMB). However, despite impressive advances, many open questions remain. Did the very early Universe undergo a phase of inflation - an accelerated expansion period in which macroscopic primordial inhomogeneities were seeded from local quantum fluctuations - and if so, what are the physical mechanisms and the fields responsible for inflation? What is the nature of the elusive dark matter and dark energy that seem to constitute more than $95 \%$ of the matter-energy density in our observable Universe? Are the apparent large-scale anomalies observed in CMB temperature maps by the WMAP and Planck space missions a signature of deviation from isotropy and homogeneity, or a statistical fluke? Is there new physics at play in the Universe, beyond the standard model of particle physics and fundamental interactions? Is the overall $\Lambda \mathrm{CDM}$ cosmological scenario correct, or are we missing an essential piece of the puzzle?

Answers to these questions can be found in additional observations of the CMB, the relic radiation that was last scattered when the Universe was about 380,000 years old and became cold enough that the primordial plasma of light nuclei and electrons combined into neutral atoms, mainly hydrogen and helium. In the process, the Universe became transparent to radiation, so that CMB photons became free to propagate. Hence when we observe them today they carry an image of the Universe at this recombination epoch, which encodes a wealth of information about the early Universe and about the interactions of CMB photons on their paths towards us. The Planck space mission has extracted most of the information 
in the primordial CMB temperature anisotropy power spectrum (Planck Collaboration et al. 2016a,e). However, the sensitivity of Planck to CMB polarisation - about $50 \mu \mathrm{K}$.arcmin (i.e., a noise level of $50 \mu \mathrm{K}_{\mathrm{CMB}}{ }^{1}$ per pixel of 1 square arcminute solid angle) - was not sufficient to extract all of the information that can be obtained from CMB polarisation. The nearoptimal exploitation of CMB polarisation signals requires measurements at the level of a few $\mu \mathrm{K}$.arcmin or better, i.e., at least an order of magnitude better than achieved by Planck.

The scientific importance of measuring CMB polarisation has stimulated a huge amount of activity in the CMB community. A number of suborbital experiments have been or are being deployed, with the objective to either detect primordial CMB polarisation $B$ modes generically predicted in the framework of inflationary models, (as recently reviewed in Ref. Kamionkowski and Kovetz 2016), or B modes due to CMB lensing (Lewis and Challinor 2006), or both. However, it is widely accepted that a space mission will be necessary to fully exploit the scientific potential of CMB polarisation.

Several concepts for next-generation space missions have already been presented in answer to calls for proposals by space agencies throughout the world. In Europe, COrE + was proposed to ESA in January 2015, but was evaluated as incompatible with the technical and programmatic boundary conditions of the M4 call, which had an unusual schedule and tight budgetary constraints. COrE + followed a previous proposal, COrE, submitted in December 2010 (The COrE Collaboration et al. 2011), and the B-Pol concept (De Bernardis et al. 2009), proposed earlier within the same programme. A French small satellite mission, the SAMPAN satellite, was proposed to CNES and underwent a preliminary feasibility study with CNES and industry in around 2006 (Bouchet et al. 2005). A Japanese satellite to study CMB polarisation, LiteBIRD, was proposed to JAXA in 2008 and is undergoing a study phase in Japan in collaboration with a team from the United States (Ishino et al. 2016; Matsumura et al. 2014). In the US, a mission concept study called EPIC/CMBpol was carried-out under a NASA contract in 2008-2009 (Bock et al. 2008, 2009), and an initial study is underway for a "Probe-class" mission currently called CMB-Probe. A different concept, PIXIE, using a Fourier transform spectrometer to observe in 400 narrow frequency bands between $30 \mathrm{GHz}$ and $6 \mathrm{THz}$ with only four bolometric detectors, has been proposed to observe not only CMB polarisation, but also measure spectral distortions of the background (Kogut et al. 2011, 2016). A comprehensive mission, PRISM, with a very broad science case, comprising both CMB polarisation and spectral distortions, was proposed to ESA in 2013 as a possible large mission, to be launched in 2028 or 2034 (André et al. 2014; PRISM Collaboration et al. 2013). None of these proposals is selected yet, but the number of proposals testifies of the strong interest of the scientific community for a future CMB space mission.

These mission concepts all propose to observe the sky at millimetre to sub-millimetre wavelengths, but differ in sensitivity (by a factor of up to 10), angular resolution (by a factor of up to 20), frequency coverage (with $\nu_{\max } / \nu_{\min }$ ranging from 5 to 200), number of detectors (from 4 to more than 10,000), number of frequency bands (from 5 to 400) and orbit (from low-Earth orbit to the Sun-Earth L2 Lagrange point). These differences arise from: mission-specific science targets; varying assumptions about the plausible level and complexity of foreground astrophysical emission and about the range of frequency bands required to clean CMB maps from astrophysical contamination; and programmatic and budgetary constraints imposed by the calls for mission concepts by space agencies, which lead to inevitable compromises.

\footnotetext{
${ }^{1}$ In CMB thermodynamic temperature units; we will drop the "CMB" subscript henceforth.
} 
In this paper, one of a series dedicated to the preparation of a post-Planck CMB space mission, we discuss the performance requirements and the possible design of a future space mission concept that will observe CMB polarisation, in order to shed new light on cosmology, and that can be implemented as an ESA medium-size mission to be launched before 2030 . This paper is part of the "Exploring Cosmic Origins (ECO)" collection of articles, each describing a different aspect of the Cosmic Origins Explorer (CORE), recently proposed to ESA in answer to the "M5" call for a medium-size space mission within the ESA Cosmic Vision Programme. We discuss the design drivers and the various options, and present the expected performance and scientific impact expected from the mission. We compare the CORE design with that of other proposals, and discuss the pros and cons of the various options. A number of relevant questions are addressed in companion papers, which investigate in more detail: the scientific case for the mission (Burigana et al. 2017; Challinor et al. 2017; CORE Collaboration et al. 2016; De Zotti et al. 2016; Di Valentino et al. 2016; Melin et al. 2017); its ability to address contamination of the observations by astrophysical foreground emission (Remazeilles et al. 2017); data analysis techniques that can help mitigate systematic effects (Natoli et al. 2017); and the design of the instrument (de Bernardis et al. 2017).

\section{Overview of CORE}

The CORE mission concept proposed to ESA in answer to the "M5" call is a polarimetric imager that will observe the sky in 19 frequency bands between 60 and $600 \mathrm{GHz}$, at an angular resolution ranging from about $2^{\prime}$ at $600 \mathrm{GHz}$ to about $18^{\prime}$ at $60 \mathrm{GHz}$. CORE is focussed on CMB polarisation, aiming at exploiting the scientific information that can be extracted from CMB polarisation $E$ and $B$ modes. One of the key science targets is the detection, precise characterisation, and scientific exploitation of CMB polarisation $B$ modes, both from inflationary gravitational waves and from the gravitational lensing of last-scattering surface CMB $E$ modes by large-scale structure along the line of sight (section 3 ). Figure 1 gives a view of how well $C O R E$ will measure $E$ and $B$ modes, and specifically primordial $B$ modes, for a tensor to scalar ratio $r$ of 0.01 or 0.001 . It also illustrates the relative importance of various sources of error in polarisation measurements, and in particular the need for accurate component separation on all angular scales to fully exploit the CMB polarisation signals over a large fraction of the sky. Indeed, over $70 \%$ of sky, Galactic foreground emission at $130 \mathrm{GHz}$ dominates over noise at all scales down to about $12^{\prime}(\ell \simeq 1000)$, and is larger than $E$-mode sample variance in bins of $\Delta \ell / \ell=0.3$ at all scales. It also dominates over lensing $B$ modes at all scales for large sky fractions. The severity of foreground contamination would be reduced if we restrict ourselves to exploiting only the cleanest part of the sky: over $5 \%$ of sky, the amplitude of foreground contamination is reduced by an order of magnitude in amplitude, so that at $130 \mathrm{GHz}$ it dominates over noise only on scales larger than about one degree. Over such a smaller patch of sky, however, cosmic variance of $E$ modes or $B$-modes is significantly increased.

The instrument uses an array of 2100 cryogenically cooled, broad-band, polarisationsensitive Kinetic Inductance Detectors (KIDs) at the focus of a 1.2-m aperture crossedDragone telescope. The full array yields an aggregate CMB polarisation sensitivity of about $1.7 \mu \mathrm{K}$.arcmin (Table 1). Frequency channels are chosen to cover a frequency range sufficient to disentangle the CMB from astrophysical foreground emission. Six frequency channels ranging from $130 \mathrm{GHz}$ to $220 \mathrm{GHz}$ are dedicated primarily to observing the CMB. The individual sensitivity of each of these channels is comparable to the level of CMB lensing, 


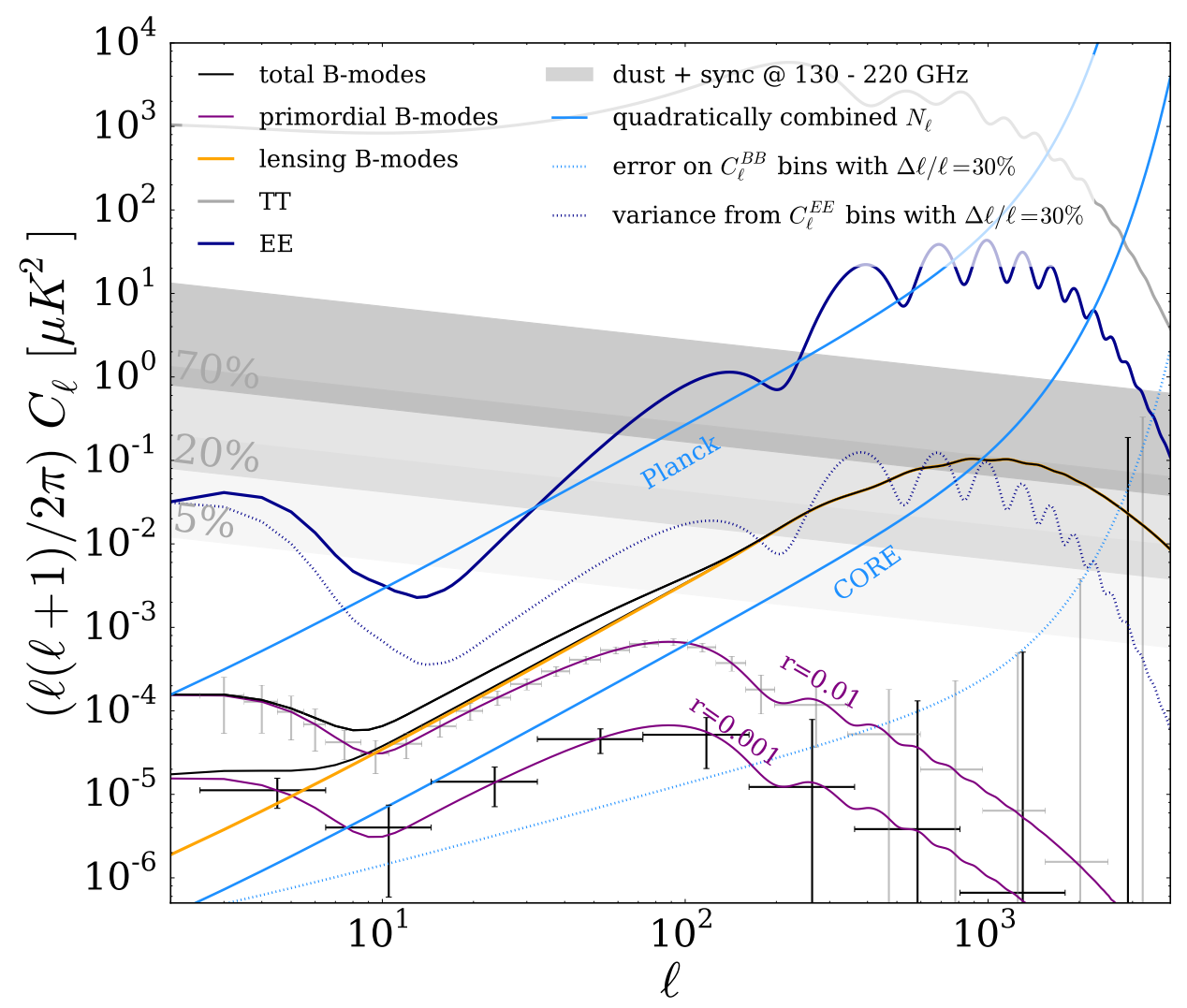

Figure 1. CMB polarisation angular power spectra $C_{\ell}^{E E}$ (dark blue), $C_{\ell}^{B B}$ from gravitational lensing of $E$ modes by large-scale structure (orange), $C_{\ell}^{B B}$ from inflationary gravitational waves $r$ (purple, for two values of the tensor-to-scalar ratio), and total $C_{\ell}^{B B}$ for $r=0.01$ (black). Two fundamental sources of error for measurements of these power spectra with CORE are shown for comparison: expected noise level (light blue); and average foreground emission over $70 \%, 20 \%$, and $5 \%$ of the sky (grey bands, from dark to light). Each of the grey bands shows the span of foreground contamination from $130 \mathrm{GHz}$ (lower limit of the band) to $220 \mathrm{GHz}$ (upper limit). Uncertainties in power spectrum estimation over bands of $\Delta \ell / \ell=0.3$ coming from $E$ modes and noise sample variance (representative of the level at which errors must be understood to take full advantage of the survey raw sensitivity) are shown as dotted lines. The error bars on the primordial $B$-mode spectra for $r=0.01$ and $r=0.001$, corresponding to $1 \sigma$ in bins ranging from $\Delta \ell / \ell \simeq 0.2$ (for $r=0.01$, at low $\ell$ ) to 0.75 (for $r=0.001$ ), illustrate the sensitivity that will be achieved for inflationary science assuming perfect component separation over $70 \%$ of sky and reduction of the contamination by lensing using small-scale CMB $E$ and $B$ modes measured by $C O R E$.

of order $5 \mu \mathrm{K}$.arcmin in polarisation. These sensitive observations at different frequencies allow for cross-comparison and cross-correlation of independent CMB maps to characterise foreground residuals and noise properties. Six channels from 60 to $115 \mathrm{GHz}$ mostly serve to monitor low-frequency and astrophysical foreground emission (polarised synchrotron, but also free-free and spinning dust in intensity, and in polarisation if required). In sky regions where synchrotron is faint these channels can contribute to CMB sensitivity as well. Seven channels ranging from 255 to $600 \mathrm{GHz}$ serve to monitor dust emission, and to map cosmic 


\begin{tabular}{|c|c|c|c|c|c|c|c|c|}
\hline $\begin{array}{c}\text { Channel } \\
{[\mathrm{GHz}]}\end{array}$ & $\begin{array}{c}\text { Beam } \\
{[\operatorname{arcmin}]}\end{array}$ & $N_{\text {det }}$ & $\begin{array}{c}\Delta T \\
{[\mu \mathrm{K} \cdot \operatorname{arcmin}]}\end{array}$ & $\begin{array}{c}\Delta P \\
{[\mu \mathrm{K} \cdot \operatorname{arcmin}]}\end{array}$ & $\begin{array}{c}\Delta I \\
{\left[\mu K_{\mathrm{RJ}} \cdot \operatorname{arcmin}\right]}\end{array}$ & $\begin{array}{c}\Delta I \\
{[\mathrm{kJy} / \mathrm{sr} \cdot \operatorname{arcmin}]}\end{array}$ & $\begin{array}{c}\Delta y \times 10^{6} \\
{\left[y_{\mathrm{SZ}} \cdot \operatorname{arcmin}\right]}\end{array}$ & $\begin{array}{c}\operatorname{PS}(5 \sigma) \\
{[\mathrm{mJy}]}\end{array}$ \\
\hline 60 & 17.87 & 48 & 7.5 & 10.6 & 6.81 & 0.75 & -1.5 & 5.0 \\
\hline 70 & 15.39 & 48 & 7.1 & 10.0 & 6.23 & 0.94 & -1.5 & 5.4 \\
\hline 90 & 12.08 & 78 & 5.1 & 7.3 & 4.19 & 1.04 & -1.2 & 4.7 \\
\hline 100 & 10.92 & 78 & 5.0 & 7.1 & 3.90 & 1.20 & -1.2 & 4.9 \\
\hline 115 & 9.56 & 76 & 5.0 & 7.0 & 3.58 & 1.45 & -1.3 & 5.2 \\
\hline 160 & 7.01 & 144 & 3.7 & 5.2 & 1.98 & 1.55 & -1.6 & 4.1 \\
\hline 175 & 6.45 & 160 & 3.6 & 5.1 & 1.72 & 1.62 & -2.1 & 3.9 \\
\hline 195 & 5.84 & 192 & 3.5 & 4.9 & 1.41 & 1.65 & -3.8 & 3.6 \\
\hline 220 & 5.23 & 192 & 3.8 & 5.4 & 1.24 & 1.85 & $\ldots$ & 3.6 \\
\hline 255 & 4.57 & 128 & 5.6 & 7.9 & 1.30 & 2.59 & 3.5 & 4.4 \\
\hline 520 & 2.29 & 96 & 116.6 & 164.8 & 1.03 & 8.56 & 8.3 & 7.4 \\
\hline 600 & 1.98 & 96 & 358.3 & 506.7 & 1.03 & 11.4 & 20.0 & 8.5 \\
\hline Array & & 2100 & 1.2 & 1.7 & & & 0.41 & \\
\hline
\end{tabular}

Table 1. Proposed CORE frequency channels. The sensitivity is calculated for a 4-year mission, assuming $\Delta \nu / \nu=30 \%$ bandwidth, $60 \%$ optical efficiency, total noise of twice the expected photon noise from the sky and the optics of the instrument being cooled to $40 \mathrm{~K}$. This configuration has 2100 detectors, about $45 \%$ of which are located in CMB channels between 130 and $220 \mathrm{GHz}$. Those six CMB channels yield an aggregate $\mathrm{CMB}$ sensitivity in polarisation of $2 \mu \mathrm{K}$.arcmin $(1.7 \mu \mathrm{K}$.arcmin for the full array). Entries for the thermal SZ Comptonisation parameter $\Delta y$ are negative below $217 \mathrm{GHz}$ (negative part of the tSZ spectral signature).

infrared background (CIB) anisotropies that can serve as a tracer of mass for "de-lensing" CMB polarisation $B$ modes (Sherwin and Schmittfull 2015). The telescope size (1.2-m aperture) is such that the angular resolution is better than $18^{\prime}$ over the whole frequency range, so that all the frequency channels can be used for component separation down to this angular resolution. In the cleanest regions of the sky, the CMB will be mapped in eight frequency channels or more, with an angular resolution ranging from $\simeq 5^{\prime}$ to $10^{\prime}$ and a sensitivity to polarisation in the $5-8 \mu \mathrm{K}$.arcmin range for each channel independently.

The geometry of the spacecraft, displayed in figure 2, is as symmetric as possible to avoid any thermal effect due to the modulation of the solar flux on the spacecraft while it spins to scan the sky. The main elements of the payload module (PLM), telescope, screens and baffles, will be kept cold by passive cooling, to minimise the requirements on the active cryogenic chain. Passive cooling of the PLM to approximately $40 \mathrm{~K}$ will be achieved by keeping the payload in the shadow of the service module (SVM), and thermally decoupling the PLM from the SVM with a set of highly reflective V-grooves (a conceptual design similar to that succesfully used on Planck, Tauber et al. 2010a), while the main payload conical screen radiates towards free space to compensate for conductive heat inflow from the SVM.

Although the design and performance of the instrument do not critically depend on the payload temperature actually achieved (which could be as high as $90 \mathrm{~K}$ or more with acceptable impact on the mission performance), the low payload temperature that is achieved by passive cooling also reduces the background on the detectors, resulting in better sensitivity overall, in particular in the frequency channels above $220 \mathrm{GHz}$.

CORE will be in orbit around the second Sun-Earth Lagrange point (L2), and will scan the sky with a dedicated scanning strategy combining a fast $\operatorname{spin}\left(T_{\text {spin }} \simeq 2\right.$ minutes $)$ around the spacecraft principal axis of symmetry, a slower precession ( $T_{\text {prec }} \simeq 4$ days) around an axis 

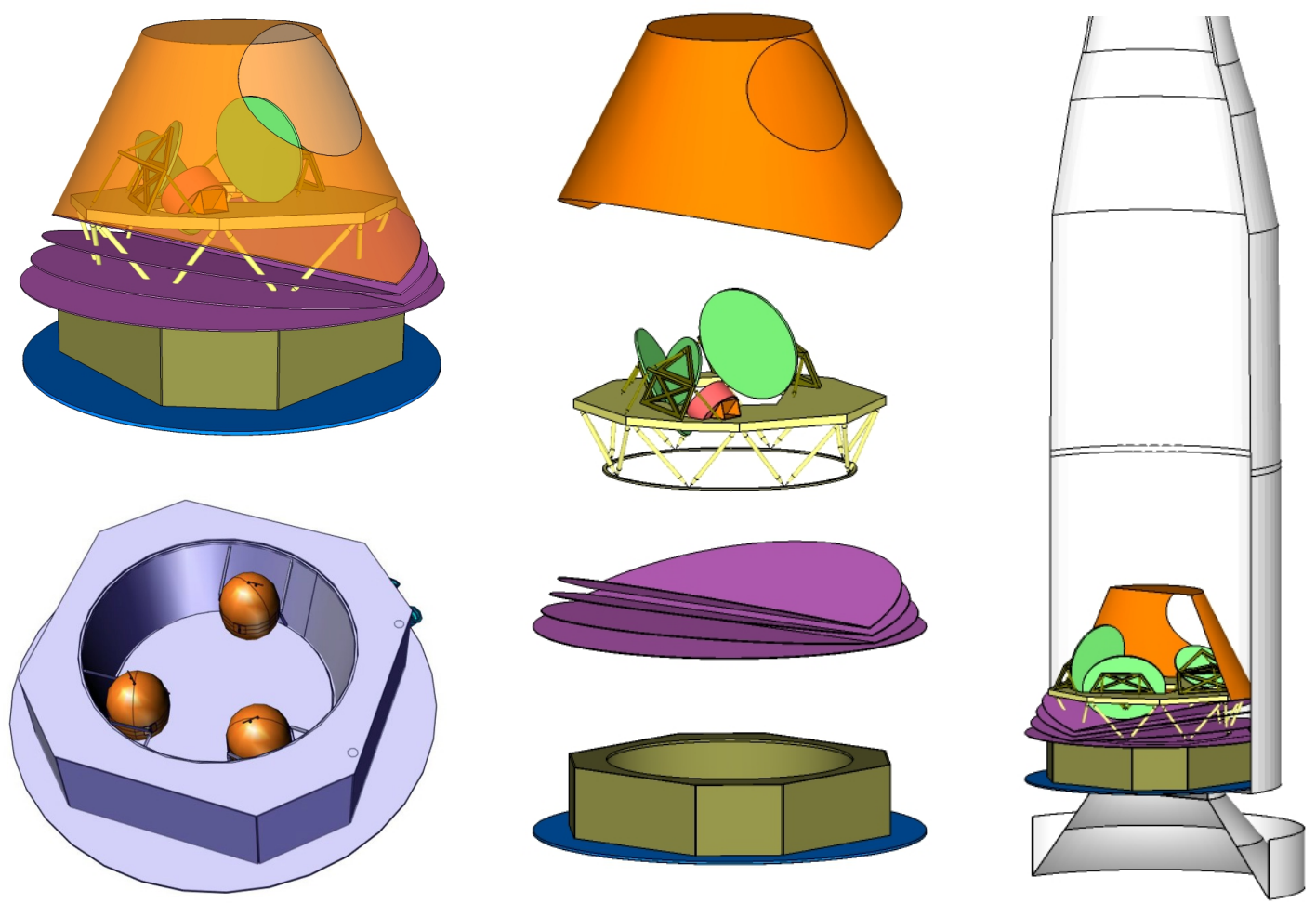

Figure 2. Baseline CORE payload and service modules. Top left: Global view of the spacecraft. Bottom left: View of the SVM following the preliminary design made by ESA in a short concurrent design facility study performed in March 2016 [http://sci.esa.int/trs/ 57795-cmb-polarisation-mission-study]. Middle: Global view of all spacecraft elements, showing the main shield (orange), the telescope (light green) on its optical bench (yellow), the focal-plane unit (FPU, red), the V-grooves (purple), and the SVM at the bottom. The FPU outer shield is not represented. Right: View of CORE in an Ariane-6.2 fairing.

that is kept anti-solar to keep the solar flux on the spacecraft constant, and a slow revolution of the whole system around the Sun with period 1 year (figure 3 ). The precession angle is $\alpha=30^{\circ}$, and the line of sight (LOS) is offset from the spin-axis by an angle $\beta=65^{\circ}$. The baseline scan strategy guarantees that each sky pixel is seen by each detector with a large number of different orientations, a property that is crucial for measuring polarisation with a good control of systematic effects. Contrarily to some mission concepts proposed earlier, the baseline version of CORE does not make use of an active polarisation modulator such as a rotating half-wave plate (HWP). Systematic effects that generate confusion between all Stokes parameters, and in particular those that result in a leakage of intensity signals into much fainter polarisation, are controlled through a combination of requirements on the instrument and on its calibration, of a scanning strategy that provides polarisation measurements and redundancies on a very large range of timescales, and of carefully constructed data-processing pipelines. Systematics are characterised and corrected for a posteriori, with a global interpretation of the scientific data themselves, marginalising over nuisance parameters that model instrument properties and sources of systematic errors. The elementary tools of the data analysis pipeline are outlined in section 7 and discussed in more detail in one of 


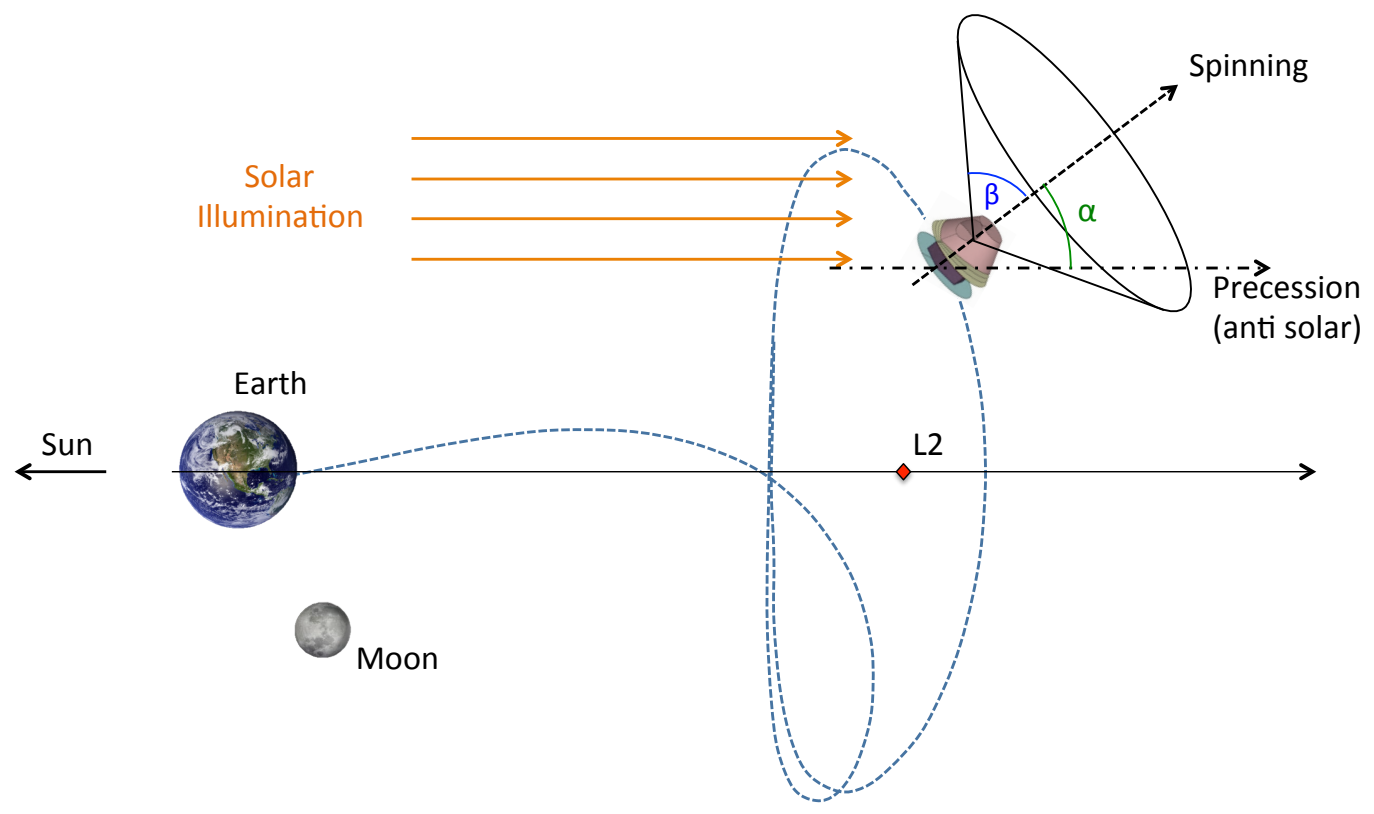

Figure 3. On an orbit around the Sun-Earth L2 Lagrange point, 1.5 million kilometre away from the Earth, the spacecraft scans the sky with three modulations of the pointing direction on various timescales. The spacecraft spins at a rate of order $f_{\text {spin }} \simeq 0.5 \mathrm{RPM}$, so that the line of sight scans the sky on quasi-circles of opening angle $\beta$ with a period of about 2 minutes. The circles are not perfectly closed by reason of a slower precession, with a period of $T_{\text {prec }} \simeq 4$ days, with precession angle $\alpha$. The precession axis is kept anti-solar, so that the symmetric spacecraft always receives the same amount of illumination from the Sun, ensuring hence the thermal stability of the payload. The last modulation is provided by the slow revolution of the whole system around the Sun with a period of one year.

the companion papers (Natoli et al. 2017).

\section{$3 \quad$ Scientific objectives}

The baseline science programme of CORE focusses on understanding the fundamental processes that gave raise to our observable Universe. This science case can be addressed with precise observations of the polarisation of the CMB. The primary science programme aims to:

1. understand the mechanisms that gave raise to primordial inhomogeneities in the very early Universe, and in particular constrain scenarios of cosmic inflation;

2 . test the standard $\Lambda \mathrm{CDM}$ model and look for possible missing pieces in our understanding of the cosmological picture;

3. look for cosmological signatures of extensions of the standard model of particles and interactions.

Additional aspects of this science programme, achievable with the same data, must be considered in order to fully exploit CMB polarisation observations. These extensions, also of major scientific interest by themselves, are: 
4. investigate and understand the cosmic structures that generate secondary CMB anisotropies superimposed on the primordial ones, in particular through the distortion of the CMB polarisation by gravitational lensing, which mixes polarisation $E$ and $B$ modes;

5. understand the astrophysical emission processes that are a source of foreground contamination for CMB polarisation observations;

6. understand the dust-obscured star-formation phase of galaxy evolution;

7. analyse cosmic dipoles in the microwave to test the isotropy and homogeneity of the Universe at the largest scales, and constrain energy dissipation processes from different cosmic epochs, including reionisation, through dipole spectrum distortions.

We now further expand the main themes of this science case, concentrating on the transformational results that will be achieved with CORE. We split the science case into five main areas: inflation; testing and constraining in detail the standard hot big-bang $\Lambda$ CDM cosmological model; constraining the standard model of particles and interactions; mapping structures in the Universe; and the legacy value of the CORE survey for other science goals.

\subsection{Inflation}

Cosmic inflation, postulated in the early 1980s to solve a number of puzzles of the standard Big-Bang (Planck Collaboration et al. 2014f, 2016d, and references therein), is the current baseline generic scenario for the generation of primordial perturbations in the early Universe. Inflationary models generically predict the existence of primordial tensor perturbations at very early times (Kamionkowski and Kovetz 2016). The amplitude of these tensor modes is parameterised with the tensor-to-scalar ratio, $r \equiv T / S$, which specifies the power of tensor perturbations relatively to that of scalar perturbations. Tensor modes (primordial gravitational waves) contribute to the total CMB temperature anisotropies and to polarisation $E$ and $B$ modes, while scalar modes (primordial density perturbations) contribute only to $T$ and $E$ modes. The detection of primordial CMB polarisation $B$ modes would provide direct evidence for cosmic inflation and for quantum fluctuations of space time, as well as determining the energy scale relevant for the inflationary epoch. Unambiguous detection of these primordial $B$ modes is hence one of the primary targets of a future CMB space mission.

A few special cases of inflation deserve special attention. The simplest models of singlefield inflation with large fields $\left(\Delta \phi>m_{\text {Planck }}\right)$ predict $r \gtrsim 0.002-0.003$ (the so-called Lyth bound, Lyth 1997). For a simple, single-field slow-roll model, an expansion in terms of slowroll parameters, $\epsilon$ and $\eta$, gives $n_{\mathrm{S}}-1=2 \eta-6 \epsilon$, while $r=16 \epsilon$. Taking Planck's measurement of $n_{\mathrm{s}}=0.9655 \pm 0.0062$ for a standard $\Lambda \mathrm{CDM}$ model, we infer that $6 \epsilon-2 \eta=0.0345 \pm 0.0062$. If $\eta \lesssim \epsilon$, we have $\epsilon \simeq 0.005-0.01$ and $r \simeq 0.1-0.2$. This scenario is already in mild tension with the current upper limit of $r \leq 0.07$ at $95 \%$ CL, coming from BICEP/Keck after foreground cleaning using WMAP and Planck data (BICEP2 Collaboration et al. 2016), but is not completely ruled out. Taking the $3 \sigma$ upper limit on $n_{\mathrm{s}}$, we get instead $r \simeq 0.05$, still compatible with present-day measurements; however, this is likely to change in the coming years, with either a detection or a clear rejection of this model.

In the case of the Starobinskii $R^{2}$ model (Starobinsky 1980), the predicted level is $r=3\left(n_{\mathrm{s}}-1\right)^{2}$ instead. The Planck constraint on $n_{\mathrm{s}}$ suggests $r \simeq 0.0035$, and $r \geq 0.0008$ for $n_{\mathrm{s}}$ at its $3 \sigma$ upper limit. This is a much bigger challenge for sub-orbital observations. Section 4.1 discusses why making a clear detection of $B$ modes at this level must be done from space. 
Lastly, we note that small field inflationary models, in general easier to connect to fundamental physics, generically predict values of $r$ smaller than the Lyth bound. Hence, $r \ll 0.001$ is as plausible a scenario as anything else (Lyth 1997). In that case, detecting primordial inflationary $B$ modes is out of reach for the foreseeable future, but nevertheless the precise and accurate observation of CMB polarisation is still a scientific necessity - even if $r \ll 0.001$, ruling out large-field models is an essential piece of information that cannot be obtained by any other means.

In the coming years, the sensitivity of ground-based CMB observatories will substantially increase. Multi-frequency observations in the atmospheric windows will improve the capability of controling foreground contamination. This evolution is likely to result in substantial improvement of the current upper limit of $r<0.07$, perhaps down to $r \lesssim 0.01$; although the contamination of the observations by Galactic foreground emission is a challenge that should not be underestimated. We require that CORE should perform at least 10 times better then this, i.e., be able to unambiguously detect or rule out $r \simeq 0.001$. The capability of detecting CMB $B$ modes to that level of $r$ - which is both well motivated scientifically and plausibly out of reach of suborbital experiments alone - is a natural science objective for a future space mission. A non-detection would rule out all large-field inflationary models. A detection would be a major discovery, and also make it possible to clearly decide between some of the currently favoured inflationary models.

Constraining inflation is not exclusively the domain of the detection of primordial $B$ modes and measurement of the value of $r$. Inflationary models are also meaningfully constrained by tightening the measurement of the spectral tilt $n_{\mathrm{s}}$ and on the variations of $n_{\mathrm{s}}$ with scale, as well as on the level of non-Gaussian signatures in the CMB maps. CORE is also designed to dramatically improve on these other inflationary observables. If primordial $B$ modes are detected, the tensor spectral index $n_{\mathrm{t}}$ also becomes an observable of interest. We refer the reader to the relevant companion paper (CORE Collaboration et al. 2016) for further details.

\subsection{The cosmological model}

Many of the main cosmological observations, such as the homogeneity and isotropy on large scales, the expansion rate, the abundance of light elements, the growth of structure, CMB temperature and polarisation anisotropies, statistics of galaxy distributions, cosmic shear measurements, supernova brightnesses, and cluster number counts, are compatible (within current uncertainties) with a $\Lambda \mathrm{CDM}$ model with just six main parameters. The remarkable agreement of this disparate set of observations with a relatively simple model also represents several big puzzles: it suggests the inflationary paradigm for the original of the initial density perturbations (as discussed above); it invokes the existence in the Universe of an unknown type of dark matter, representing roughly $25 \%$ of the total matter-energy density; and it also requires the existence of the even more mysterious dark energy, accounting for about $70 \%$ of the energy content in the Universe at present, and responsible for the observed acceleration of the expansion and for the dilution of large-scale structures at late times. Clues about the exact nature of both "dark" components are lacking, leaving room for many possible options, as well as for imaginative theoretical speculation.

In addition, in spite of a remarkable overall concordance, some apparent tensions exist between the model and subsets of the data. Although currently near the limits of statistical significance that would be required to seriously challenge the standard cosmological model, these tensions add to a sense of unease in postulating that our Universe is filled at the $95 \%$ 
level with forms of matter and energy that are completely unknown. As an example, the Hubble constant today inferred by Planck from the CMB at the last-scattering epoch (Planck Collaboration et al. 2016e) is discrepant at about the $2.4 \sigma$ level with Hubble Space Telescope Cepheid+SNe-based estimates (Riess et al. 2011). These recent values are consistent with the earlier tension noted in the first Planck cosmological parameters paper (Planck Collaboration et al. 2014d), which generated some debate in the community. Another intriguing discrepancy is found between the value of the amplitude $\sigma_{8}$ of density perturbations at the scale of $8 h^{-1} \mathrm{Mpc}$ inferred from cluster number counts (Planck Collaboration et al. 2016g) and the value inferred from the $\mathrm{CMB}$ alone. Additional discrepancies, at a lower level of significance have been suggested through the inferred amount of lensing in the CMB angular power spectrum, via differences in sub-sets of CMB data, in the curvature $\Omega_{k}$ and in other specific parameters (Addison et al. 2016; Couchot et al. 2017; Planck Collaboration 2016g). So how can we determine if these tensions are more than just statistical fluctuations?

The CMB is currently the key observable for quantifying this global cosmological picture. CMB photons probe the Universe at the earliest possible times and on the largest possible scales. The CMB is also the unique backlight that shines on all structures between the last-scattering surface at $z \simeq 1100$ and observers on Earth at $z=0$. The complete exploitation of the information it carries is a scientific imperative for cosmology (Galli et al. 2014; Scott et al. 2016). With high S/N maps of $T, E$ and $B$, yielding cosmic-variance dominated measurements of the temperature and polarisation angular power spectra $C_{\ell}^{T T}, C_{\ell}^{T E}$, $C_{\ell}^{E E}$, and angular power spectrum $C_{\ell}^{\phi \phi}$ of the CMB lensing potential, errors on cosmological parameters as currently best constrained with the CMB by Planck (Planck Collaboration et al. 2016e) can be reduced by factors that can reach an order of magnitude or more (Di Valentino et al. 2016). Such a drastic improvement will clarify whether existing tensions are an indication of a departure from the standard cosmological scenario, a statistical excursion, or a systematic error in one of the measurements. New tensions that are undetectable as of now are also likely to be uncovered - when considering extensions to the standard cosmological scenario, the total volume of the error, represented by the figure of merit

$$
\mathrm{FoM}=\left(\operatorname{det}\left[\operatorname{cov}\left\{\Omega_{\mathrm{b}} h^{2}, \Omega_{\mathrm{c}} h^{2}, \theta, \tau, A_{\mathrm{s}}, n_{\mathrm{s}}, \ldots\right\}\right]\right)^{-1 / 2}
$$

computed from the covariance matrix of the errors on a set of cosmological parameters, can be improved by a factor as much as $10^{7}$, depending on the extensions considered. This improvement in constraining the cosmological scenario is essential for making progress on the current puzzles. We refer the reader to Ref. (Di Valentino et al. 2016) for an in-depth discussion regarding future constraints on cosmological parameters with CORE alone, as well as in combination with other cosmological data sets.

Finally, improving the determination of the CMB dipole amplitude and direction and comparing it with analagous investigations in other wavebands, which exploit signals from different types of astrophysical sources, probing different shells in redshift, provide an important test of fundamental principles in cosmology. The extension of boosting effects to polarization and cross-correlations with CORE will enable a more robust determination of purely velocity-driven effects that are not degenerate with the intrinsic CMB dipole, allowing us to achieve an overall signal-to-noise ratio close to that of an ideal cosmic variance limited experiment up to a multipole $l \simeq 2000$ significantly improving on the Planck detection. We refer the reader to Ref. (Burigana et al. 2017) for further discussion. 


\subsection{Fundamental particles and interactions}

The standard model of particles and interactions is remarkably successful at describing the fundamental laws of nature. Families of elementary particles, which constitute the building blocks for all of the experimentally observed forms of matter, as well as the carriers of the known interactions between them, have been identified and their main characteristics have been determined. However, this model is incomplete.

First and foremost, there exists at present no model that unifies the force of gravity with the other known forces of nature. The coupling constant for gravity is so small that the gravitational interaction cannot be probed on the scale of individual particles (the ratio of gravitational to electric interaction between an electron and a proton is of the order of $\left.10^{-40}\right)$. Gravity can only be probed with massive objects, for which all other interactions are effectively screened by factors of at least $10^{40}$. Hence, the cosmos is an essential laboratory for understanding the laws of physics when gravity is taken into account.

Even if one ignores gravity, the standard model of particle physics is still incomplete for a number of other reasons. For instance, the standard model does not currently explain why neutrinos have mass, while the observation of neutrino oscillations implies a non-vanishing difference of squared mass for the different eigenstates, i.e.,

$$
\Delta m_{12}^{2} \simeq 7.5 \times 10^{-5} \mathrm{eV}^{2}
$$

and

$$
\left|\Delta m_{13}^{2}\right| \simeq 2.5 \times 10^{-3} \mathrm{eV}^{2},
$$

but do not constrain the absolute mass scale of the neutrinos (Nakamura and Petcov 2016).

Measuring CMB lensing, $C_{\ell}^{\phi \phi}$, breaks parameter degeneracies and enables estimates to be made for the sum of the neutrino masses (e.g., Kaplinghat et al. 2003). The precise predictions depend on details of the neutrino sector (e.g., whether they have the normal or inverted mass hierarchy) and on what other data are used in combination. However, one conclusion of Ref. (Di Valentino et al. 2016) is that CORE, together with Euclid and DESI should provide $\sigma\left(M_{\nu}\right)=16 \mathrm{meV}$, yielding a $4 \sigma$ detection of the neutrino mass sum.

Accurate measurements of CMB polarisation can also constrain additional neutrino species or other light relics. This is parameterised by the quantity $N_{\text {eff }}$, which has the value 3.046 in the standard model (slightly higher than 3 because of details of neutrino decoupling). The expected uncertainty is $\sigma\left(N_{\text {eff }}\right)=0.041$ from CORE alone, and $\sigma\left(N_{\text {eff }}\right)=0.039$ in combination with future BAO data (Di Valentino et al. 2016).

There are many other directions in which physics beyond the standard model can be constrained with a sensitive CMB polarisation survey such as planned with CORE. This includes: dark matter annihilation and decay; variation of fundamental constants; topological defects; and signatures of stringy physics.

\subsection{Structures}

Much of cosmic history is probed by observations of the growth of structures after the last scattering of CMB photons. A space mission dedicated to precision CMB polarisation science will also trace the growth of cosmic structures using three independent probes: CMB lensing; galaxy clusters; and the cosmic infrared background. 
Lensing: Gravitational lensing by large-scale structures along the path of the CMB photons slightly distorts the anisotropy and polarisation patterns of the primordial CMB (Lewis and Challinor 2006). This gravitational lensing effect mixes $E$ and $B$ modes, giving rise to lensing $B$ modes on all angular scales. $B$ modes due to the lensing of $E$ modes into $B$ modes peak at $\ell \simeq 1000$, i.e., angular scales of order 10 arcminutes. Their amplitude on larger scales is similar to that of white noise of amplitude $5 \mu \mathrm{K}$.arcmin. For $r<0.01$, lensing $B$ modes dominate $B$-mode polarisation at all scales except the very largest ones $(\ell<10)$, and they dominate over the noise in the error budget for detecting primordial $B$ modes when maps reach a noise level of order $5 \mu \mathrm{K}$.arcmin. Hence, a space mission attempting to observe $r \lesssim 0.01$ will also inevitably observe CMB lensing, and have to deal with the corresponding contamination, which degrades the sensitivity to primordial $B$ modes.

CMB lensing, however, is not only a nuisance for measuring inflationary $B$ modes; it also is a unique observable for probing the full distribution of matter between us and the lastscattering surface at $z \simeq 1100$, i.e., in the whole observable Universe, in a way that does not rely on baryonic tracers and does not require us to understand non-linear growth effects in detail. It is a way of directly observing the distribution of dark matter and hence is a primary goal for future CMB observations. CMB lensing effects have already been detected by Planck and by several ground-based experiments (e.g., Planck Collaboration et al. 2016f). These clear detections however, still have limited signal-to-noise ratio per pixel, and/or limited skycoverage. A future $B$-mode survey can transform this area of research, providing accurate maps that can be used for precision cosmology and cross-correlation with large-scale structure surveys.

Clusters: Galaxy clusters, detectable in the frequency range of interest for CMB observations, distort the CMB spectrum via the thermal Sunyaev-Zeldovich (tSZ) effect, which is interaction of the hot intracluster gas with CMB photons through inverse Compton scattering (Carlstrom et al. 2002). Clusters are a particularly sensitive probe of the growth of cosmic structure (e.g., Planck Collaboration et al. 2014e). By measuring the abundance of clusters as a function of redshift, we can tightly constrain the dark energy equation of state and the neutrino mass scale, and look for deviations to standard gravity theory. Doing this requires accurate and precise calibration of the cluster mass-observable scaling relations, which in turn requires good lensing measurements of cluster masses out to redshifts $z>1$. A CMB temperature and polarisation survey can calibrate the normalisation of the SZ signalto-mass scaling relation using CMB halo lensing. To obtain enough clusters and calibrate their scaling relation to sufficient accuracy requires a survey covering a large sky fraction with angular resolution comparable to the scale of clusters, and high sensitivity in temperature and polarisation.

For the baseline survey, we expect that CORE will detect tens of thousands of galaxy clusters, with several hundred at redshifts $z>1.5$. The cluster sample will extend to higher redshifts than the eROSITA catalogue and will be a critical resource for studies of galaxy formation in dense environments, especially when coupled with NIR surveys such as those from Euclid and WFIRST. Using CMB lensing measurements towards detected clusters, the normalisation of the SZ signal-to-mass relation can be calibrated to the percent level at $z<1$, and to better than $10 \%$ at redshifts approaching $z=2$. Under these conditions and in combination with primary $\mathrm{CMB}$ constraints, a large cluster catalogue will tightly constraint the dark energy equation of state. Moreover, with enough sensitivity and frequency coverage a cluster survey will enable: studies of the relativistic SZ effect by stacking hundreds of 
clusters; extraction of cluster pairwise momentum at signal-to-noise $>70$; and measurement of the evolution of CMB temperature with redshift to test the standard model. Even if galaxy clusters are not considered a design-driver for CORE, joint analysis of CORE and CMB-Stage 4 (CMB-S4) data sets will push the detection mass limit towards $3 \times 10^{13} \mathrm{M}_{\odot}$ and increase the cluster yield by a factor of 4 over either experiment alone, including at the higher redshifts (Melin et al. 2017).

Gas, stars and dust: One of the major research trusts in modern cosmology is the understanding of the relative distributions of luminous stars, diffuse gas and dark matter (e.g., Guo et al. 2010). In particular, we need to understand how baryons cool to form stars and are reheated by feedback in a cycle that must be finely tuned to allow less than $10 \%$ of baryons to end up in stars. This is a central question in galaxy formation studies and a critical element for interpreting stage 4 dark energy programmes. The stage 4 lensing surveys rely on percent-level predictions for the total matter distribution, but feedback can modify the matter distribution much more than this. New avenues of research in this area will be opened by observing the distribution of the gas, through both the tSZ and the kinetic SZ (kSZ) effects, as well as the total matter distribution through CMB lensing. Even if, again, this science topic is not a design driver for CORE, the wide frequency coverage that is needed for CMB polarisation science also is essential to extract an all-sky tSZ map that accurately separates the signal from foregrounds, especially the CIB anisotropies that limited the Planck result. In complement to the large galaxy samples from planned imaging and spectroscopic surveys (e.g., Euclid, WFIRST, LSST, DESI, and PFS), a space mission that maps CMB lensing, the tSZ effect, and the CIB will measure for the first time the relative distribution of galaxies, gas and total matter out to redshifts beyond the peak of cosmic star formation at $z \simeq 2$. The CIB measurements will also trace star-formation activity and dust production at critical epochs around the peak epoch of star formation. A future survey such as proposed with CORE will substantially improve on Planck the characterisation of CIB fluctuations in both temperature and polarization and will use the frequency dependence of CIB dipole to reduce by at least one order of magnitude the uncertainty of absolute CIB spectrum currently provided by COBE/FIRAS. Even if not design-drivers of the CORE mission, all these measurements represent unique capabilities of CORE to address key questions in the development of structure.

\subsection{Legacy}

CORE is a space mission with the ability to produce well-characterised maps of the complete sky in the $60-600 \mathrm{GHz}$ frequency range, with both very high sensitivity and good angular resolution compared to existing data. As such, the mission's data set can also be used to answer scientific questions beyond the primary CMB science objectives described above, to an extent that depends on the extent to which each of these areas can be considered as a design driver.

In particular, the need to monitor Galactic foreground contamination for CMB science is illustrated in figure 1. For a sky coverage of $\simeq 70 \%$, foreground emission is the dominant source of error for all $\ell \lesssim 1000$, i.e., at all angular scales larger than about $12^{\prime}$. It also is above the full-sky cosmic variance of $E$ modes in multipole bins of $\Delta \ell / \ell=0.3$, on all scales. While observing in the cleanest few per cent of sky for the first detection of primordial $B$ modes might be possible, the full exploitation of CMB $E$ modes and of lensing $B$ modes requires observations over a substantial fraction of the sky to avoid loss of sensitivity (because of 
cosmic variance). Hence, observing foreground emission on all relevant angular scales is required. This opens up the opportunity to investigate the role of the Galactic magnetic field in structuring the Galactic interstellar medium (as has started to be done using Planck data, Planck Collaboration 2016a,b,c,d,e,f; Planck Collaboration et al. 2016b,c).

Magnetism is a facet of our cosmic origins that observations have yet to uncover in any detail. Magnetic fields are not observable directly but they may be studied by observing polarised radiation. Early attempts have shown the existence of coherent magnetic fields on all observed scales from proto-planetary discs to clusters of galaxies, but current data are too limited to reveal the processes that have amplified and organised the much weaker primordial field, and to unveil the role that magnetic fields play in the formation of galaxies, stars and planets (e.g., Parker 1979).

Cosmic magnetism is a rapidly-advancing topic across astrophysics. The Planck all-sky dust polarisation map was a spectacular highlight of that mission, which has revealed the fingerprints of the Galactic magnetic field on interstellar matter (Planck Collaboration et al. 2016a). ALMA is driving a new revolution where magnetic fields will be imaged along the star-formation sequence from pre-stellar cores to proto-stars and their proto-planetary disks. Over the coming decade, stellar polarisation combined with Gaia astrometry should yield a 3D model of the magnetic field of the Milky Way on Galactic scales. Further in the future, the SKA will extend our horizon further, probing magnetic fields in distant galaxies, clusters, and the cosmic web, while CORE will offer unprecedented statistics on dust polarisation from the Galaxy to characterise the interplay between gravity, magnetic fields and turbulence in cosmic space.

Polarisation observations provide an opportunity to study magneto-hydrodynamical (MHD) turbulence and dynamo action in great detail within our Galaxy. What can be learned from CMB experiments on dust polarisation will complement advances expected from Faraday tomography measurements with lower frequency telescopes like LOFAR, eVLA, ASKAP, and SKA (e.g., Van Eck et al. 2017). The detection potential for relevant plasma processes and their characteristic scales, like those of turbulent energy injection and dissipation, can be increased considerably via the sensitivity and statistics expected from a future CMB polarisation space mission, which, in order to monitor foreground contamination, must necessarily map dust polarisation with an unprecedented combination of sensitivity and angular resolution.

Dust and synchrotron radiation from the Galaxy provide complementary views of interstellar magnetic fields. Synchrotron radiation traces magnetic fields over the whole volume of the Galaxy, while dust polarisation traces them largely within the disk, where interstellar matter is concentrated and stars form. The statistical properties of Galactic magnetic fields are imprinted on those observables and methods to extract this information from observational data have started to be developed. Quantities highly relevant for an understanding of Galactic turbulence and dynamo processes, such as the energy, helicity, and tension force spectra, have been shown to be encoded in synchrotron intensity, polarisation, and Faraday rotation measures. Likewise, the analysis of the Planck data has prompted a number of studies that are relating dust polarisation to the magnetic-field structure and its interplay with the density structure of matter (Planck Collaboration et al. 2016a). Since dust sub-mm emission is an optically-thin tracer of all ISM components (neutral, atomic and molecular, and ionised), dust polarisation is best-suited to investigate the magnetised interstellar matter, in particular the formation of its filamentary structure, and within filaments the initial conditions of star formation. 
Due to our location within the Galaxy, the fluctuations at a given angular scale in observables correspond to magnetic-field structures of different physical sizes. Disentangling these in order to identify physical scales and processes is a challenge, which calls for a statistical approach. The leap forward in statistics of CORE compared with Planck (a factor of a few hundred in the number of measured modes) will greatly enhance our ability to identify signatures of the processes involved in MHD turbulence, in particular coherent magnetic-field structures associated with localised dissipation of turbulent energy. What will be learned from these data will complement what will be probed by ground-based telescopes (e.g., ALMA and SKA) observing dust polarisation from compact sources and Faraday rotation. Together, these projects will have a major impact on our understanding of the role of magnetic fields in galaxy and star formation.

Extragalactic sources are also a potential contaminant of CMB observations. Highredshift, dusty galaxies can be observed at sub-millimetre wavelengths with angular resolution better than that of Planck, which did not have diffraction-limited angular resolution in its three highest frequency channels. A full-sky survey, such as that of CORE, would detect thousands of strongly lensed (and hence extremely bright) high- $z$ galaxies distributed over the full sky, which can then be studied in extraordinary detail through follow-up observations. Also, CORE can be used to detect high-redshift proto-clusters beyond the reach of surveys in other frequency bands. CORE will also detect the polarised emission from thousands of individual radio sources and dusty galaxies. These science objectives are further discussed in a companion paper (De Zotti et al. 2016).

The observation of the background of unresolved high-redshift dusty galaxies that form the CIB, an essential tool for delensing CMB $B$ modes and detecting low-level primordial $B$ modes, also open up the possibility of further studying cosmological star formation, as discussed by Ref. (Wu and Doré 2016). Finally, precise analyses of the dipole spectrum over a wide frequency range give us the chance to significantly improve with respect to COBE-FIRAS in the recovery of CMB spectral distortion parameters for both early and late dissipation processes, from a factor of several up to about 50 (or even much better for an ideal experiment with the CORE configuration), depending on the quality of foreground removal and relative calibration, allowing us to detect, for example, the energy release associated with cosmological reionisation.

\section{Survey requirements}

Starting from main scientific objectives, we now discuss how the mission design stems from the survey requirements and goals, in terms of overall sensitivity, angular resolution, and channels of observation. In this section, we assume that we want to extract essentially all the cosmological information encoded in CMB polarisation only, with the space survey alone. Down-scope options stating the requirements for similar CMB polarisation performance in combination with ground-based observations, as well as interesting up-grade options for extra science in addition to CMB polarisation, are discussed in section 8.

\subsection{The need for a space mission}

It is reasonable to ask how much of the above CMB polarisation science programme can plausibly be done from the ground? Plans for a very sensitive ground-based CMB experiment, CMB-S4, are being actively made, with a science case that covers many of the topics discussed above (Abazajian et al. 2016). The strawman design for an ambitious ground-based CMB-S4 
programme targets a CMB sensitivity of the order of $1 \mu \mathrm{K}$.arcmin and angular resolution of $1-3^{\prime}$ at $150 \mathrm{GHz}$, with a sky fraction of around $50 \%$ (spread over regions with various levels of Galactic foreground contamination).

With such sensitivity and angular resolution, in the absence of additional sources of error, CMB-S4 would outperform a space mission such as CORE, for which the sensitivity of the full array is $1.7 \mu \mathrm{K}$.arcmin, for an angular resolution in the $5-10^{\prime}$ range. However, coming back to the sources of error displayed in figure 1, we note the following important issues.

- Over the cleanest $70 \%$ of the sky, foreground emission dominates over noise for all $\ell \lesssim 1000$; hence, all scales larger than about $12^{\prime}$ should be observed at multiple frequencies in order to reduce foreground contamination and achieve noise-limited observations.

- Over the same sky fraction, foreground emission dominates over $B$ modes for all multipoles; again, efficient component separation will be needed on all scales to observe $B$ modes (both primordial and lensing) with noise-dominated performance.

- Foreground residuals after component separation will be difficult to characterise, and are hence a source of potential bias. For such residuals to be below noise and/or cosmic variance uncertainties in bins of $\Delta \ell / \ell=0.3$, foreground contamination must be reduced by at least 3 orders of magnitude in amplitude at $\ell \simeq 10,2$ orders of magnitude at $\ell \simeq 100$, and 1 order of magnitude at $\ell \simeq 1000$; this is unlikely to be doable with groundbased experiments, which must thus exploit only significantly cleaner, and hence much smaller sky regions. With the reasonable assumption that only half or less of the $50 \%$ sky observed from the ground can be safely used for precision cosmology, a groundbased survey can at most exploit the CMB on $\lesssim 25 \%$ of the sky.

- The cosmic variance of full-sky $E$ modes dominates over noise for all $\ell \lesssim 2500$. For cosmological constraints based on polarisation $E$ modes, it is hence preferable to increase the size of the survey, rather than to observe smaller patches deeper; this is best done from space, with enough channels for accurate monitoring of the foreground emission.

- The cosmic variance of full-sky lensing $B$ modes dominates over the noise for all $\ell \lesssim 1000$. Hence, again, for cosmological constraints based on polarisation lensing $B$ modes, it is preferable to increase the size of the survey, rather than to observe smaller patches deeper; in addition, the confusion between primordial and lensing $B$ modes dominates the error on primordial $B$ modes for all scales below $\ell \simeq 1000$. Space offers the opportunity to accurately map the CIB, for $B$-mode delensing by a factor of $2-3$ over a large fraction of the sky; for a quick comparison, CIB-based delensing by a factor of $2-3$ over $70 \%$ of sky is as efficient at reducing the cosmic variance of residual lensing as CMB-based delensing by a factor of $5-8$ over $10 \%$ of sky (which requires $\sqrt{7}$ times better delensing to compensate for the reduced sky fraction).

For all these reasons, when considering multipoles up to $\ell \simeq 1000-2000$, the performance of future CMB observations for exploiting CMB polarisation power spectra will be limited not by raw detector sensitivity, but by the capability of removing foreground contamination and by the capability to separate lensing $B$ modes from primordial $B$ modes over the largest solid angle. A space mission with sufficient sensitivity and angular resolution is vastly superior to ground-based observatories for controlling these main sources of error over a large fraction of the sky. 
We now discuss in more detail some of the key issues with ground-based observations, so that we can make a realistic assessment of the capability of ground-based programmes to reach the CORE science targets.

\subsubsection{Atmosphere}

CMB temperature and polarisation anisotropies are best observed in the frequency range extending from a few tens to a few hundreds of $\mathrm{GHz}$, i.e., at wavelengths ranging from about $1 \mathrm{~mm}$ to about $1 \mathrm{~cm}$, around the peak of the CMB 2.725-K blackbody emission. In this frequency range, ground-based observations are possible in a set of windows through which the atmosphere is sufficiently transparent. The main atmospheric windows are centred around minima of atmospheric emission at about $30,90,150$, and $220 \mathrm{GHz}$. The transmission at $60^{\circ}$ elevation is of order $99 \%$ at $30 \mathrm{GHz}, 98 \%$ at 90 and $150 \mathrm{GHz}$ and of order $96 \%$ at $220 \mathrm{GHz}$ from the Atacama plateau, when the amount of precipitable water vapour is at the level of $0.5 \mathrm{~mm}$ (at Llano de Chajnantor in Chile, the observing conditions are better than that about $25 \%$ of the time). Even in these atmospheric windows, the atmosphere contributes to the total photon background and hence the photon noise, so that the mapping speed of a space-borne instrument is at least 100 times better than on the ground, for an identical number of detectors (see appendix A).

Even more problematic than background loading, fluctuations of atmospheric emission due to inhomogeneities in temperature or water vapour content generate strong parasitic signals, and are a source of unstable calibration (because of varying airmass and opacity), i.e., from the ground, the CMB is observed through a shiny and fluctuating curtain of atmospheric absorption and emission. In the best CMB channels for ground-based observations (90 and $150 \mathrm{GHz}$ ), about $75 \%$ of the time the atmosphere above one of the best observing sites on Earth (the Atacama plateau) is more than $2 \%$ emissive, i.e., contributes a background of more than $6 \mathrm{~K}$. As the telescope scans the sky, it scans through inhomogeneities of this emission. Even at a level as low as $0.1 \%$ (easily achieved with fluctuations of air temperature and/or water content at about the same order of magnitude), one gets $6 \mathrm{mK}$ of spurious large-scale signal or more, correlated between focal-plane detectors. A more detailed model of atmospheric turbulence gives fluctuations in the $15-30 \mathrm{mK}$ range for the best $25 \%$ of the observing time (Errard et al. 2015). At a scale of around $2^{\circ}$ this atmospheric signal is about 6 orders of magnitude larger than the 8 -nK raw sensitivity of a $1 \mu \mathrm{K}$.arcmin survey. Since scanning the same patch $10^{12}$ times is not a realistic option, this signal must be removed by a combination of processing, e.g., filtering, exploitation of multi-frequency or multi-detector observations with analysis methods such as those discussed in Refs. (Delabrouille et al. 2002) or (Patanchon et al. 2008), and polarisation modulation with a rotating half-wave-plate. Current observations demonstrate that polarisation modulation can reduce this signal by $2-3$ orders of magnitude in amplitude. Even then, residuals are still at a challenging 3 orders of magnitude above the target sensitivity on $2^{\circ}$ angular scales. The situation is even worse at larger scales and/or at higher frequencies.

A space mission completely avoids the complexity of atmospheric absorption, emission, and fluctuations. More details about the atmosphere can be found in Appendix A.

\subsubsection{Astrophysical foregrounds}

CMB observations must address the problem of astrophysical foreground emission. At frequencies below about $100 \mathrm{GHz}$ CMB observations are contaminated by a complex mixture of low-frequency astrophysical sources of electromagnetic radiation that include Galactic 
synchrotron, free-free, and anomalous dust emission (presumably spinning dust, or possibly magnetic dust, or both), as well as numerous extragalactic radio sources, while at frequencies above about $100 \mathrm{GHz}$ thermal dust emission is the dominant foreground. At frequencies above approximately $200 \mathrm{GHz}$, in addition to thermal dust emission, anisotropies of the cosmic infrared background (CIB), and to a lesser extent zodiacal-light emission dominate the fluctuations of the observed sky brightness over most of the sky. Molecular lines, notably those of carbon monoxide at multiples of $115 \mathrm{GHz}$, clearly seen in Planck data (Planck Collaboration et al. 2014a), must also be taken into account (as well as those of isotopologues at nearby frequencies, ${ }^{13} \mathrm{CO}$ and $\mathrm{C}^{17} \mathrm{O}$ near multiples of $110 \mathrm{GHz}, \mathrm{C}^{18} \mathrm{O}$ near multiples of $112 \mathrm{GHz}$ ). Several lines of $\mathrm{CO}$ emission, at about 220, 225 and $230 \mathrm{GHz}$, are located in one of the main atmospheric windows.

Of all of those foregrounds, synchrotron is the one that is known to be the most polarised (in theory, up to 75\%). At high frequency, only thermal dust is known to be very clearly polarised ( $\gtrsim 10 \%)$. Other sources of emission can be somewhat polarised (at a level $\lesssim 1 \%$ ).

While for temperature fluctuations there are regions where CMB signals strongly dominate over astrophysical foregrounds, this is not the case for polarisation (see figure 1). To remove foreground contamination, it is necessary to observe the sky at several frequencies and exploit the fact that the emission law of the CMB is substantially different from that of most foreground emission processes (Delabrouille and Cardoso 2009). Exploiting these colour differences is best done by combining observations in a set of well chosen different frequency bands. COBE-DMR observed the sky in three different frequency bands, WMAP in five, and Planck in nine. To exploit the largest possible fraction of the sky at a sensitivity level at least an order of magnitude better than Planck, even more frequency bands will be required.

Although the details will be known only with observations at the appropriate level of sensitivity, i.e., with future CMB data themselves, a simple accounting argument suggests that no less than ten channels are required, and preferably more. The two main known polarised Galactic emission sources in the frequency range of interest are synchrotron and thermal dust. To model the synchrotron (parameterised in each sky pixel by intensity, spectral index, and possible curvature of spectral index), at least three low-frequency channels are required, four to provide some redundancy, or even more if one has to model synchrotron emission with more than one simple emission law per pixel. The same is true for thermal dust at high frequency, for which at least three parameters per pixel are needed to adjust a model with a single modified blackbody emission law. Four channels at least are needed to make this measurement, with an extra channel for a consistency check. This means that a total of eight channels are needed to model the foreground emission if only synchrotron and dust must be taken into account. On top of this, thermal dust emits with more than one population of grains so it is possible that a single modified blackbody is not sufficient for a model that is accurate at the $\lesssim 1 \%$ level. Finally, the CMB itself must be observed in at least two frequency bands that are sufficiently distant in frequency for a useful cross-check, which is essential to detect possible residual foreground emission, and preferably more bands to understand the origin of any discrepancy (Planck effectively used comparisons between the CMB seen in four channels, at 70, 100,143, and $217 \mathrm{GHz}$, to investigate foreground residuals and systematic errors). The conclusions is that ten channels is the absolute minimum to monitor foreground emission in polarisation.

There is no way for this number of frequency channels, which must be well spread over the useful frequency range, to be accommodated in only four atmospheric windows. While 
synchrotron can in principle be observed from the ground at a few frequencies $\nu \lesssim 30 \mathrm{GHz}$, thermal dust emission, which dominates at frequencies where the observing conditions from the ground are poor, must be mapped from space (or, as an intermediate solution, from stratospheric balloons, which, however, have significant residual atmospheric noise, less flexibility for choosing the observing strategy, and much reduced observing time compared with a space mission).

\subsubsection{Systematic effects}

Very precisely controlling systematic effects due to non-idealities in the instrument, which are a potential major source of error for future sensitive CMB observations, is mandatory for achieving the science goals of future CMB polarisation observations. Instrumental imperfections include complex response of the instrument to external radiation or stimuli. Such non-idealities impact the shape of the response in time, in space (beams), or in frequency (bandpass); in practice these can be different from the design specifications and can be mismatched between detectors. There can also be gain fluctuations, susceptibility to events that are not related to the observations (such as cosmic ray hits), magnetic susceptibility, variations of the observing environment that impact the detector response, etc. To minimise such effects, space offers unmatched observing conditions, in an extremely stable environment. This allows for:

- minimising sidelobe pickup of emission from the Earth, Sun, and Moon;

- minimising thermal fluctuations of parts of the instrument that are optically coupled to the detectors;

- avoiding fluctuations of the response of the instrument, which is essential for enabling the calibration of instrumental imperfections at the level of accuracy required to correct for their impact on the CMB science;

- redundancy on different timescales without need for specific re-pointing, nor changing the observing conditions;

- maximal sky coverage, allowing for redundant analyses exploiting independently several large regions of sky.

This is essential because the impact of systematics can be minimised and accurately assessed only through analyses which require enough redundancy and a stable instrument.

\subsubsection{Why space - summary}

Sub-orbital CMB observations have been key pathfinders in CMB science, from the initial detection of the CMB more than fifty years ago to now. However, it also is true that all the major steps forward have been achieved by space missions. COBE-DMR, WMAP, and Planck have all been transformational for cosmology: $C O B E$ confirmed the blackbody spectrum of the CMB, ruling-out alternatives to the hot Big-Bang scenario, and detected the first temperature anisotropies that were required to explain the origin of structures; WMAP set the stage for precision cosmology; and Planck, in turn extracted essentially all cosmological information available in the CMB TT spectrum, provided today's reference maps of CMB temperature and polarisation, of CMB lensing on large scales, and of polarised emission of astrophysical foregrounds that contaminate the CMB signal (Planck Collaboration et al. 2016a). 
Data from CMB space missions have also been essential for planning and analysing CMB observations made with ground-based instruments. The sensitive polarisation observations on degree-scales made from the ground with BICEP2 and the Keck array have used the highquality Planck CMB temperature map for calibration, and for systematic effect corrections. Planck's observations have also been essential to assess the level of dust in the BICEP2 detection of $B$-mode polarisation. Thinking a decade into the future, high-quality CMB $E$ mode polarisation maps, as well as $E$ - and $B$-mode foreground maps from space missions, will also be essential for the interpretation of deep observations of CMB polarisation from the ground on selected patches of the sky.

There are good reasons why the best observations of CMB temperature anisotropies have been carried out from space, starting with the first detections with $C O B E$-DMR to the precision cosmological picture brought by Planck. Polarisation is more challenging, and must be done from space. Ground-based observations will serve as a technological roadmap, and for observing the small scales that are too costly from space. Along the way, the sub-orbital programme may bring some breakthroughs, perhaps even including a first tentative detection of primordial $B$ modes. Irrespective of what happens, however, the next polarisation space mission should be designed to wrap up everything and to deliver CMB polarisation data sets that will be the reference for decades to come. Just as Planck did for temperature anisotropies before, CORE is designed with this objective in mind for polarisation.

\subsection{What survey?}

The range of options for a future space mission is quite wide, as illustrated by the diversity of existing proposals. The main drivers of the design of the survey we propose are: (i) guaranteed scientific breakthrough for the science targets discussed above (at the time the mission delivers its scientific results); (ii) maximum science versus cost, within the programmatic and budgetary constraints of an ESA M-class mission; (iii) focus on the science objectives and observations that are out of reach from the ground, with a priority on precision CMB polarisation science; (iv) within practical constraints, achieve a performance that is limited by fundamental and astrophysical constraints (e.g., the CMB cosmic variance, the possibility to separate foregrounds in practice, and the possibility to separate inflationary $B$ modes from lensing $B$ modes) rather than by the instrument, so that the mission is "near ultimate" (in the sense that another CMB polarisation mission will not be required after CORE). The main parameters defining the mission, further discussed in the next subsections, are:

- sky coverage (section 4.3), for best sensitivity on the largest scales and for reducing the part due to cosmic variance in the determination of $E$ - and $B$-mode power spectra;

- sensitivity and angular resolution (section 4.4), which determine the capability of the mission to detect $E$ and $B$ modes with sufficient $\mathrm{S} / \mathrm{N}$ over the required range of angular scales;

- frequency range of observations and number of frequency channels (section 4.5), which govern the ability of the experiment to separate CMB observations from other sources of astrophysical emission;

- strategy for modulating polarisation and controlling systematic effects (section 4.6), in particular those arising from the confusion between CMB temperature anisotropies and CMB polarisation. 


\subsection{Sky coverage}

CORE must observe the CMB over the largest possible sky fraction to accurately measure the largest angular scale $E$ and $B$ modes, where the reionisation bumps in the CMB polarisation power spectra are located $(\ell \lesssim 10)$. It is essential for the mission to detect both the reionisation and recombination bumps of the primary $\mathrm{CMB}$ in order to confirm the inflationary origin of any detected $B$ modes. The largest scales are also essential for measuring the reionisation optical depth $\tau$ from $E$-type polarisation, and to lift the degeneracy of $\tau$ with the sum of neutrino masses. On the largest angular scales, one can also check whether anomalies detected in temperature maps by WMAP and Planck (Bennett et al. 2011; Eriksen et al. 2004; Planck Collaboration et al. 2014g) are also visible in polarisation maps. Since measuring these large scales from the ground is very challenging (if possible at all), a space mission is the only way to obtain this essential piece of information.

Considering also that at all angular scales the cosmic variance depends on the inverse of the sky fraction, we must seek to observe a large solid angle (e.g., about $50 \%$ of clean CMB sky or more) so that error bars on the measured spectra are not increased much (e.g., not by more than $\sqrt{2}$ ) by reason of cosmic variance. Given this constraint, and considering the legacy value of the observations, it is logical (but not strictly required) to plan that a CMB space mission should observe the complete sky, as was the case for all other CMB space missions before. The sampling variance being minimal for homogeneous survey depth, we also initially require the sky coverage to be as uniform as possible (the same observation time in all pixels), although this last requirement can be relaxed with better overall mission sensitivity.

However, if $r \lesssim 10^{-3}$, a tentative detection of primordial $B$ modes will require very accurate delensing and foreground subtraction. If the errors turn out to be dominated by foreground residuals it would make sense (for the particular objective a detecting very low primordial $B$ modes) to seek the best possible sensitivity on the cleanest $10-20 \%$ of sky that will also be observed from the ground, to ultimately combine observations with both the many frequency channels provided by space (for foreground monitoring), and the high angular resolution in atmospheric windows provided by the ground (for optimal delensing using the CMB only). We should hence envisage the capability to concentrate observing time on selected patches at the end of the mission in the case that primordial $B$ modes still escape detection by then. Although not a strict requirement, this capability could also be used for non-CMB science on targets of interest, such as Galactic dust filaments, interesting galaxy clusters, or deep fields observed by other instruments, for an extension of the mission's science harvest.

\subsection{Sensitivity and angular resolution}

To obtain all of the information from CMB polarisation, it is necessary to measure with good $\mathrm{S} / \mathrm{N}$ all of the CMB $E$ and $B$ modes. Figure 4 compares the final error on polarisation $C_{\ell}$ for a noise level of $2 \mu \mathrm{K}$.arcmin (sufficient for measuring lensing $B$ modes with $\mathrm{S} / \mathrm{N} \simeq 2.5$ per mode in amplitude) with a beam ranging from $2^{\prime}$ to $32^{\prime}$. This illustrates the modes that are lost when the angular resolution is reduced.

$E$ modes: For a beam of $4^{\prime}$ or smaller, $E$-mode observations are signal dominated almost up to $\ell \simeq 3000$. When the beam gets bigger, the $E$-mode measurement becomes progressively more degraded, so that for a $32^{\prime}$ beam, the future mission would not do better than Planck for $\ell \gtrsim 700$ (the exponential cut-off of the Gaussian beam yields a very rapidly raising error 


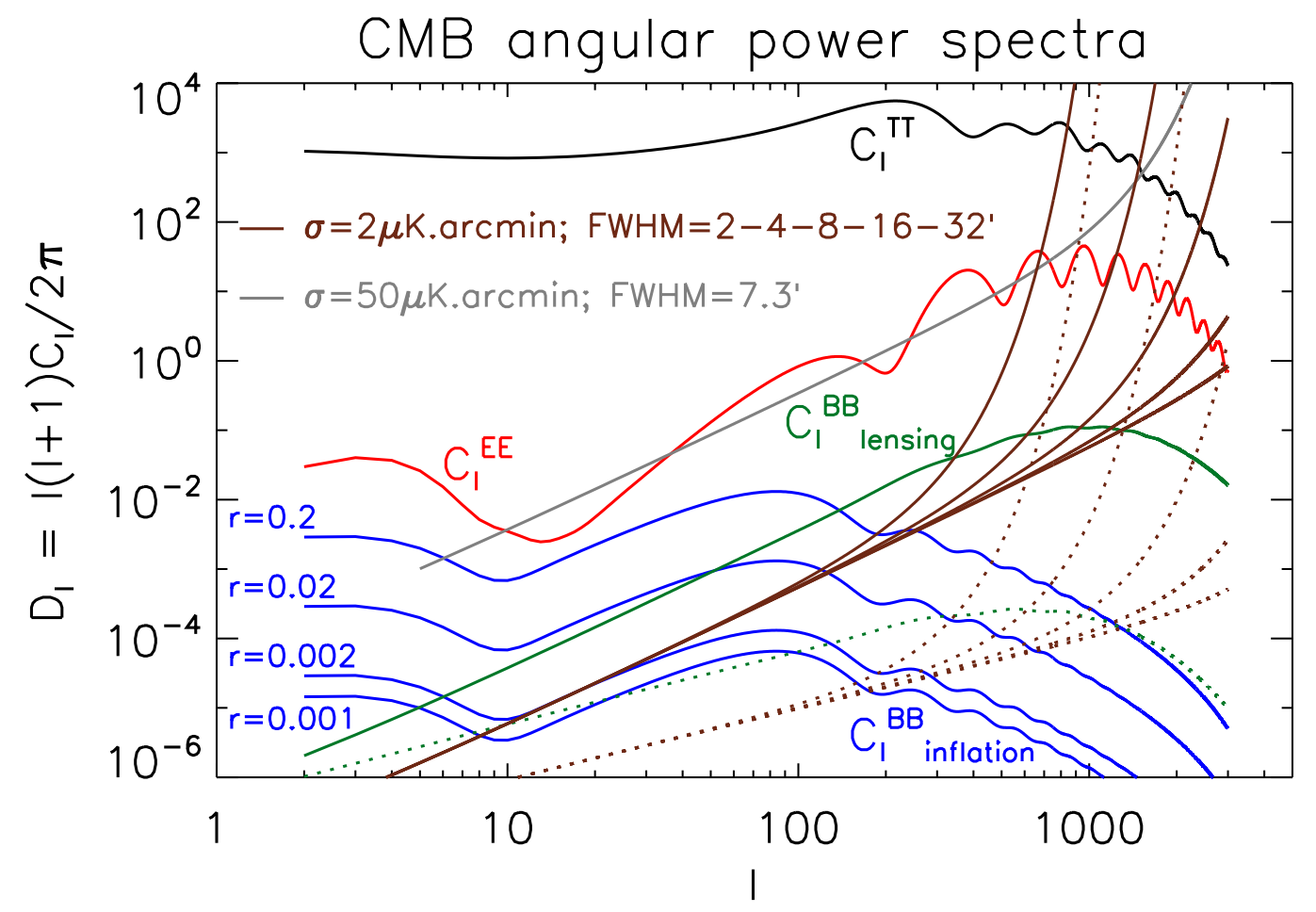

Figure 4. CMB temperature and polarisation angular power spectra. The grey line corresponds to the spectrum of instrumental noise representative of the polarisation sensitivity achieved with the Planck mission, at the level of $50 \mu \mathrm{K}$.arcmin for a beam of $7.3^{\prime}$. While Planck has observed most of the CMB temperature anisotropies with a high signal-to-noise ratio (better than inferred from the figure, which shows the polarisation sensitivity), $E$ modes have been observed with $S / N \sim 1$, and the level of lensing $B$ modes has been detected only statistically. Solid brown lines show the level of noise for a map sensitivity of $2 \mu \mathrm{K}$.arcmin and a beam of $2^{\prime}, 4^{\prime}, 8^{\prime}, 16^{\prime}$, or $32^{\prime}$ (from bottom to top), and dotted brown lines correspond to the error on $C_{\ell}$ for the same noise level and angular resolution, after smoothing by $\Delta \ell=0.3 \ell$. A full-sky observation with angular resolution of $8^{\prime}$ or better would detect lensing $B$ modes (green line) with $S / N=2.5$ per individual mode up to $\ell \simeq 1000$. For $r>0.002$, both the recombination bump (peaking at $\ell \simeq 80$ ) and the large-scale reionisation bump can be detected in bins of $\Delta \ell=0.3 \ell$. The error, however, is dominated by the cosmic variance of the $B$-mode lensing spectrum in such bins (dotted green). Unambiguously detecting the recombination bump at the level of 0.001 requires reducing by a factor of $\simeq 3$ the contamination by lensing $B$ modes (delensing).

at high $\ell$ ). Hence, the ultimate $E$-mode measurement requires a noise level $\lesssim 2 \mu \mathrm{K}$.arcmin and angular resolution $\lesssim 4^{\prime}$.

\begin{tabular}{|c|c|c|c|c|c|c|}
\hline Beam & $2^{\prime}$ & $4^{\prime}$ & $8^{\prime}$ & $16^{\prime}$ & $32^{\prime}$ & $64^{\prime}$ \\
\hline \hline$b_{\ell}^{2}$ for $\ell=80$ & 1.000 & 0.998 & 0.994 & 0.975 & 0.904 & 0.667 \\
$b_{\ell}^{2}$ for $\ell=200$ & 0.998 & 0.990 & 0.961 & 0.855 & 0.534 & 0.081 \\
$b_{\ell}^{2}$ for $\ell=1000$ & 0.941 & 0.783 & 0.378 & 0.020 & 0.000 & 0.000 \\
\hline
\end{tabular}

Table 2. Impact of the beam on $C_{\ell}^{B B}$ over the first $B$-mode recombination bump, at $\ell=80$ and $\ell=200$, and at the peak of the lensing $B$ modes $(\ell=1000)$, for various angular resolutions. 
Inflationary $B$ modes: For primordial $B$ modes, under the assumption that the lensing contamination can be completely removed by other means (external data sets such as the CIB, futuristic matter surveys, or lensing maps from very accurate small-scale CMB polarisation observations), dotted brown lines in figure 4 show that $2 \mu \mathrm{K}$.arcmin seems adequate to detect $\mathrm{r}=0.001$. However, we also require that the mission provides a means of delensing $B$-mode maps, so that the lensing residual does not exceed much the noise level of the mission.

The angular resolution of the final CMB map should be such as not to reduce the effective sensitivity to the recombination bump, which peaks at $\ell \simeq 80$ and extends up to $\ell \simeq 200$ (higher-order bumps are well below the noise level for $2 \mu \mathrm{K}$.arcmin noise, irrespective of the angular resolution). Table 2 gives the effective beam damping, $b_{\ell}^{2}$ at $\ell=80$ and $\ell=200$ for various beam sizes. Covering that range of multipoles is important to confirm the inflationary origin of the observed signal. A beam of $64^{\prime}$ clearly is very sub-optimal, since it degrades the sensitivity to primordial $B$ modes across the recombination peak, from a factor of $2 / 3$ at $\ell=80$ to a factor of more than 10 at $\ell=200$. With a loss of power ranging from $2.5 \%$ to $14.5 \%$ only between $\ell=80$ and $\ell=200$, a beam of $16^{\prime}$ is completely adequate. A beam of $32^{\prime}$ is adequate at $\ell=80$ (10\% power loss), but marginal at $\ell=200$ (47\% power loss).

Lensing $B$ modes: The shape of the lensing $B$-mode spectrum is such that the lensing power is measured almost equally well with $4^{\prime}$ or $8^{\prime}$ angular resolution, for a detector noise level of $2 \mu \mathrm{K}$.arcmin, so that to improve the measurement of the lensing power spectrum, better sensitivity is more important than better angular resolution. A signal-to-noise ratio per mode of $2-3$ (in amplitude) is achieved with a sensitivity in the $1.7-2.5 \mu \mathrm{K}$.arcmin range. Table 2, however, also gives the impact of the beam on the $B$-mode lensing power at $\ell=1000$, and shows that for fully exploiting the lensing $B$ modes, an angular resolution $\lesssim 4^{\prime}$ is desirable. An $8^{\prime}$ beam reduces the CMB power at $\ell=1000$ by a factor close to 3 , and a beam significantly larger than about $8^{\prime}$ does not resolve the lensing $B$ modes anymore. This is in line with the capability to map the lensing potential as a function of the sensitivity and angular resolution of the survey, discussed in Ref. (Hu and Okamoto 2002). Their figure 4 shows that the level of the noise in the reconstructed lensing map decreases until the angular resolution is about $2^{\prime}-4^{\prime}$ and the noise level in polarisation maps is about $0.1-0.3 \times \sqrt{2} \mu \mathrm{K}$.arcmin.

Noting that ground-based observations can make accurate small-scale CMB observations to complement the space mission data in the atmospheric windows, we can assume that they might measure the smallest CMB scales on at least a fraction of sky if the space mission fails to do so. It is necessary, however, that the space mission provide a matching angular resolution of the order of $2^{\prime}-4^{\prime}$ at frequencies $\gtrsim 300 \mathrm{GHz}$, which are hard to observe from the ground, to monitor dust contamination at all scales. A space mission that fulfills this requirement also provides an angular resolution of order $6^{\prime}-12^{\prime}$ in $\mathrm{CMB}$ channels between 100 and $200 \mathrm{GHz}$.

Other science: Improving the angular resolution of the space mission would be beneficial for non-CMB science, in particular for cosmology with clusters (Melin et al. 2017), for observing extragalactic sources (De Zotti et al. 2016), and for investigating Galactic magnetism with polarised dust emission on small scales. However, complementarity with sub-orbital observations can also be used for some of these science goals, which are not primary drivers of the CORE mission concept.

Summary of sensitivity and angular resolution requirements: In summary, the space survey should be designed to provide, after component separation, a clean CMB map 

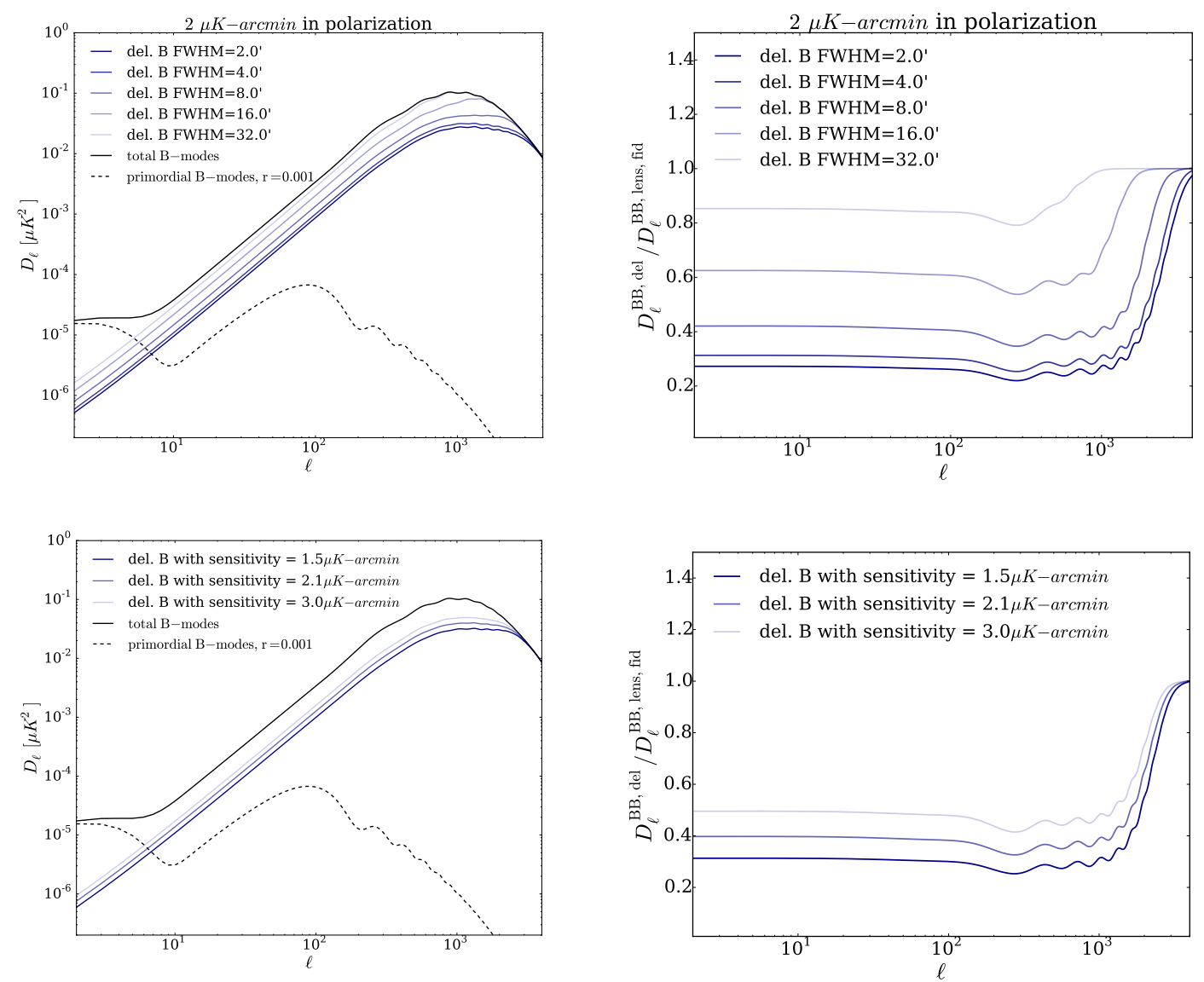

Figure 5. Effectiveness of CMB $B$-mode polarisation delensing for varying sensitivity and angular resolution assumptions. Top: Delensing effectiveness for $2 \mu \mathrm{K}$.arcmin noise and various beam sizes. The left panel shows the level of residual lensing, compared to primordial $B$ modes for $r=0.001$, and the right panel shows the ratio of residual lensing power over initial lensing power; a beam of $4^{\prime}$ is requested for removing $2 / 3$ of the lensing contamination. Bottom: Delensing effectiveness for the CORE optical configuration, and three different sensitivity assumptions.

over more than $50 \%$ of the sky with at least:

- sensitivity in the 1.7-2.5 $\mu \mathrm{K}$.arcmin range for a signal-dominated $B$-mode map (with a signal-to-noise ratio of $2-3$ on the lensing $B$ modes);

- CMB angular resolution better than about $30^{\prime}$ for detecting primordial $B$ modes;

- CMB angular resolution $\lesssim 8^{\prime}$ for exploiting most of the detectable lensing $B$-mode spectrum;

- CMB angular resolution $\lesssim 4^{\prime}$ for optimal measurement of polarisation $E$ modes and lensing $B$ modes, as well as for separating lensing from primordial $B$ modes.

The actual performance of different surveys for CMB polarisation science is discussed in more detail in companion papers (Challinor et al. 2017; CORE Collaboration et al. 2016; Di Valentino et al. 2016).

We also note that to provide a foreground-cleaned CMB map at a given resolution, all the channels used for foreground cleaning must have at least that resolution. For primordial 
$B$-mode science at the level of $r \simeq 10^{-3}$, both the synchrotron and the dust should be mapped with an angular resolution of about $30^{\prime}$ or better. For primordial $B$-mode science with $r \lesssim 0.01$, signal dominated primordial $B$-mode maps at $\ell \gtrsim 10$ also require delensing, by a factor of about 3 in power for $r=0.003$, and about 10 for $r=0.001$. In addition, for $r=0.001$, figure 4 shows that the cosmic variance of the lensing signal in bins $\Delta \ell / \ell=0.3$ is at about the level of the $B$-mode recombination bump, so that delensing is required to achieve a detection. For a noise level of $2 \mu \mathrm{K}$.arcmin, the required delensing by a factor of 3 in power can be achieved with CMB polarisation if the survey has an angular resolution of about $4^{\prime}$ (see figure 5).

Alternatively, delensing can be achieved with precise maps of the CIB, and the capability to delens with both small-scale CMB polarisation and the CIB would provide a useful crosscheck, as well as better delensing overall. Hence, in addition to the above CMB surveys, for enabling "ultimate" CMB polarisation science, possibly in combination with ground-based observations reaching an angular resolution of about $2^{\prime}$, the space survey must also provide maps of high-frequency foregrounds at a matching angular resolution. This leads to the following additional requirements:

- dust polarisation maps with an angular resolution of about $2^{\prime}$, for component separation on small scales;

- CIB intensity maps for an alternative way of $B$-mode delensing, essential for detecting primordial $B$ modes below $r \simeq 0.01$.

These high-frequency observations must be done from space. On the other hand, small-scale synchrotron polarisation maps, which are too challenging to obtain from space because of the required telescope size, can be obtained with large ground-based radio telescopes if necessary.

\subsection{Frequency channels}

We distinguish requirements for the main CMB science (addressed with the observation of CMB polarisation $E$ and $B$ modes) and for other science goals.

CMB polarisation: The minimum of foreground emission relative to the CMB is located between 60 and $100 \mathrm{GHz}$. One might then think that the best frequency to observe CMB polarisation would be located precisely at this minimum. However, there are practical advantages to observing at higher frequencies.

- Since the beam size of a diffraction-limited telescope scales as the inverse of the frequency, the angular resolution that can be achieved at 120 and $180 \mathrm{GHz}$ is, respectively, 2 and 3 times better than at $60 \mathrm{GHz}$.

- When CMB observations are made at $150 \mathrm{GHz}$ or above, most of the complex lowfrequency foreground emission signals are low enough that it suffices to reduce their contamination by a factor $\lesssim 10$. Only dust contamination needs to be accurately subtracted (at the sub-percent level) from the CMB polarisation observations.

- This dominant astrophysical foreground can be monitored at $\nu>200 \mathrm{GHz}$ with better angular resolution than that of the CMB channels, while for a single dish multifrequency instrument, low-frequency foregrounds are observed at lower angular resolution, and hence cannot be monitored over the full range of useful angular scales.

- Finally, as shown in Appendix A, the CMB sensitivity per focal-plane area in space is maximal in the $150-250 \mathrm{GHz}$ frequency range. 
Taking this into account, we should observe the CMB mostly in bands centred between about 130 and $200 \mathrm{GHz}$, avoiding the $\nu=115 \mathrm{GHz}$ and $\nu=230 \mathrm{GHz} \mathrm{CO}(J=1 \rightarrow 0)$ and $(J=2 \rightarrow 1)$ lines. We also need channels below and above this frequency range for monitoring foreground emission. A factor of around 2 in frequency provides proper leverage for distinguishing the various emission processes, i.e., we need frequency bands extending from about 65 to $400 \mathrm{GHz}$. Ground-based telescopes can provide synchrotron observations over large patches of sky at $\nu \lesssim 40 \mathrm{GHz}$, so observing those frequencies with a space-based mission is not a priority. High frequencies, however, must be observed from space.

As discussed above in section 4.1.2, at least 10 different channels in that frequency range, and preferably more, are required to separate the different foreground emission signals from the CMB over a substantial fraction of sky. Additional bands are required to extend the fraction of sky that can be used for CMB science. Spreading frequency channels logarithmically in the $65-400 \mathrm{GHz}$ frequency range with frequency ratios such that $\nu_{n+1} \simeq 1.15 \nu_{n}$ (a sampling in frequency well matched with a bandwidth $\Delta \nu / \nu$ of approximately $30 \%$ per channel) yields a set of 15 frequency channels that is adequate for CMB science, with some safety margin. Actual CORE channels are obtained by a similar process (starting at $\nu_{0}=60 \mathrm{GHz}$ instead of $65 \mathrm{GHz}$, and making the channels a bit closer in the main CMB frequency range).

Further optimisation depends on assumptions about polarised foreground emission properties. To be on the safe side, it is preferable to pick a baseline with more channels than strictly required, in case polarised foreground emission turns out to be more complicated than current models might suggest.

Other science: We now consider options to further optimize the legacy value of the survey, in particular in combination with complementary ground-based CMB observations with CMB-S4.

Accurate science with galaxy clusters requires a frequency range covering both the minimum and the maximum of the thermal SZ distortion, i.e., ranging from around $120 \mathrm{GHz}$ to $400 \mathrm{GHz}$. A few channels in that frequency range (at least three, but preferably more for redundancy) are required to separate the thermal SZ from the kinetic SZ and also to monitor temperature effects, which, for hot clusters, can modify the thermal SZ spectrum by $5-10 \%$. These requirements are fulfilled with the channels selected on the basis of CMB polarisation science.

Additional channels above and below this frequency range are required to monitor contamination by radio and/or infrared sources in clusters, and to avoid confusion with smallscale foreground emission, in particular Galactic dust and the CIB. Again, a factor of around 2 in frequency below $120 \mathrm{GHz}$ and above $400 \mathrm{GHz}$ seems adequate, with at least four channels on both sides. This suggests extending the frequency range to span $60-800 \mathrm{GHz}$, with four channels above $400 \mathrm{GHz}$ and four channels below $120 \mathrm{GHz}$. A 220-GHz channel, close to the zero of the thermal SZ effect, separates CMB anisotropies and the kinetic SZ from the thermal SZ (assuming that the CO lines are controlled in some way, e.g., with notch filters). For the best synergy with the future CMB-S4 programme, the angular resolution of the high-frequency channels above $\nu \simeq 300 \mathrm{GHz}$ on the space mission must match that of the ground-based survey at 150 and $220 \mathrm{GHz}$.

CIB anisotropies are useful as a delensing tool for primary CMB science. As demonstrated with Planck, mapping the CIB anisotropies is relatively easy in patches with low dust emission, using channels at 350, 550 and $850 \mathrm{GHz}$. At higher frequency, low-redshift infrared galaxies, observable by other means, start to dominate the CIB emission, and dust emission 
from our own galaxy is relatively stronger. With at least five channels above $300 \mathrm{GHz}$ (but preferably more for increased accuracy), it will be possible to use a generalised internal linear combination method (Remazeilles et al. 2011) to separate CIB emission from thermal dust over a significant fraction of sky, as demonstrated using Planck and IRAS observations (Planck Collaboration et al. 2016h). The CIB map obtained in this way, in addition to its intrinsic scientific interest, can be used to delens CMB $B$ modes and improve constraints on the primordial CMB $B$-mode spectrum, independently of what can be done with CMB delensing only. This is an essential tool for cross-checking and validating delensing based on CMB data only.

A principal component analysis can be used on multi-channel CIB observations to infer the star-formation rate (SFR) as a function of redshift. A study of how well different future experiments would perform (Wu and Doré 2016), showed that the constraints steadily improve with the number of channels between 220 and $850 \mathrm{GHz}$, provided sufficient angular resolution is available. From their figure 3, a factor of 30 is gained on the SFR figure of merit they define by increasing the number of channels above $200 \mathrm{GHz}$ from 5 to 10 , and yet another similar factor is gained with 25 channels above $200 \mathrm{GHz}$. Although not designed for CIB science, with eight channels above $200 \mathrm{GHz}$, CORE outperforms other experiments such as LiteBIRD (which in addition suffers from reduced angular resolution) and CMB-S4 (with frequency bands limited to atmospheric windows). CORE could be further optimised for CIB science; this could be considered at a later stage if compatible with budgetary and programmatic constraints of the implementation of the mission.

\subsection{Systematic effects}

Polarising bolometric detectors integrate the electromagnetic power along one polarisation axis. They measure a combination of intensity and linear polarisation Stokes parameters. Assuming an infinitely small beam and frequency band, the signal on the detector, once it is calibrated in units of CMB temperature fluctuations, can be modelled as

$$
x=I+\eta(Q \cos (2 \psi)+U \sin (2 \psi))+n,
$$

where $I$ is the sky brightness and $Q$ and $U$ are the two Stokes parameters describing linear polarisation in the observed sky direction and at the relevant frequency, $\eta$ is polarisation efficiency (ideally equal to unity), $n$ is the noise term, and $\psi$ is an angle of observation with respect to a set of reference axes. $E$ and $B$ polarisation are obtained by non-local linear combinations of $Q$ and $U$ (Kamionkowski et al. 1997; Zaldarriaga and Seljak 1997). In order to measure $Q$ and $U$, it is necessary to invert a linear system of measurements at different orientations of the form

$$
x_{i}=I+\eta\left(Q \cos \left(2 \psi_{i}\right)+U \sin \left(2 \psi_{i}\right)\right)+n_{i},
$$

which can be rewritten in vector-matrix format

$$
\boldsymbol{x}=\mathrm{A} s+\boldsymbol{n},
$$

where $s=[I, Q, U]$ is the vector of sky Stokes parameters, and $\mathrm{A}$ is an operator that integrates the pixel and frequency-dependent sky signal $s(p, \nu)$, and can be thought of as a generalised mixing or pointing matrix that depends on detector responses (calibration, polarisation efficiency, beams, and frequency bands) and on the scanning and observing strategy 
(pointing, orientation, and polarisation modulation). If an estimate $\widehat{A}$ of the mixing matrix A is known, an estimate of $s$ is obtained by linear inversion as

$$
\widehat{s}=\left[\widehat{A}^{\top} W \widehat{A}\right]^{-1} \widehat{A}^{\top} W x
$$

and the best estimate of $s$ in the least squares sense is obtained when the "weighting matrix" $\mathrm{W}$ is the inverse of the noise covariance matrix of the observations.

Inserting $\boldsymbol{x}=\mathrm{A} \boldsymbol{s}+\boldsymbol{n}$ in eq. (4.4) we immediately see that if $\widehat{\mathrm{A}}=\mathrm{A}$, and if there are enough measurements in each sky pixel (with different scanning angles) for the matrix $\widehat{A}^{\top} W \widehat{A}$ to be invertible, the estimate of $s$ is unbiased, since $\widehat{s}=s$ up to an additive noise term that does not depend on $s$. If, however, $\widehat{A} \neq \mathrm{A}$, we obtain

$$
\widehat{s}=\left[\widehat{A}^{T} W \widehat{A}\right]^{-1}\left[\widehat{A}^{\top} W A\right] s+\text { noise }
$$

where the product of the first two matrices (in square brackets) is different from the identity. Non-vanishing off-diagonal terms generate mixing between $I, Q$, and $U$. Since $I \gg E \gg B$, the most problematic effects are leakage of intensity into polarisation, and leakage of $E$ into $B$.

The survey should allow for the inversion of the system in eq. (4.3) accurately enough that the total uncertainty on the final CMB data products is dominated by detector noise at the required level of $2 \mu \mathrm{K}$.arcmin. Instrumental imperfections that generate systematic errors, and ways to mitigate them by design and during the data analysis phase, are further discussed in section 7, as well as in a dedicated companion paper (Natoli et al. 2017).

\subsection{Flexibility, safety margins, and redundancy}

The survey requirements above are somewhat approximate (by choice). Indeed, the scientific capabilities of CORE are continuous functions of the main parameters defining the survey, so there is no reason to be too prescriptive in the specific value of a particular parameter. For example, although full-sky coverage is preferable, the main goals of the mission are not sacrificed if a fraction of sky is missing, up to a few tens of percent if the missing part is in regions of high Galactic emission. Additionally, although it is highly desirable to map the CMB with a sensitivity sufficient for signal-dominated $B$-mode mapping, the ability of the mission to reach its science goals is not radically different if the signal-to-noise ratio per mode (or pixel) is only $\simeq 2$ or $\simeq 3$ (even if, of course, the higher the better). Similarly, there is some flexibility in the exact angular resolution that needs to be achieved - whether primordial $B$ modes are mapped with $30^{\prime}$ angular resolution or $20^{\prime}$ is not absolutely critical, nor whether lensing $B$ modes are mapped with $4^{\prime}$ or $6^{\prime}$ angular resolution. The impact of the mission characteristics on the scientific performance of CORE is studied more rigorously in the companion papers (Challinor et al. 2017; CORE Collaboration et al. 2016; Di Valentino et al. 2016).

In spite of this flexibility, we must realise that the task is challenging. As can be seen in figure 1, the primordial $B$-mode power spectrum, $C_{\ell}^{B B}$, must be detected at a level that is $4-6$ orders of magnitude below the foreground contamination $C_{\ell}$, an order of magnitude below the lensing $B$-mode spectrum, 8 orders of magnitude below $C_{\ell}^{T T}$ and 3 orders of magnitude below $C_{\ell}^{E E}$. It is not sufficient to design the mission to just barely be able to detect a small excess of power that could be primordial $B$ modes (assuming that foreground and lensing contamination as well as systematic effects will be under control). Even with a design that 
is optimised to avoid such contamination, the ability must be built in for ascertaining that the desired signal is what is being measured. This calls for safety margins and redundancy in the capacity to detect primordial $B$ modes.

The lensing $B$-mode power spectrum, $C_{\ell}^{B B}$, although larger and hence easier to detect than primordial $B$ modes for $r \lesssim 0.01$, as already seen in existing results (Keisler et al. 2015; The Polarbear Collaboration: P. A. R. Ade et al. 2014), is nevertheless challenging to accurately measure. The power spectrum at its peak is 4 orders of magnitude below $C_{\ell}^{T T}, 2$ orders of magnitude below $C_{\ell}^{E E}$, and at about the level of the foreground contamination over most of the sky. Detecting these $B$ modes is one thing, but measuring them precisely enough that the uncertainty on $C_{\ell}^{B B}$ is dominated by the sample variance of Gaussian noise, or by their own cosmic variance, is another thing entirely. Systematic effects and foregrounds must be controlled at the proper level.

The survey's ability to detect and scientifically exploit $B$ modes must have built-in redundancy. After the analysis of the data from the mission, the only way to convince oneself that an excess $B$-mode signal al low $\ell$ is indeed a detection of primordial $B$ modes is to show that the signal persists when the data are split into parts. One can ask if the signal is seen equally well in each of the first and second halves of the mission? In the north and south parts of the sky? With different Galactic cuts? In separate frequency channels, after component separation with different methods? For such tests to be implemented, the survey must have extra frequency channels, and be capable of detecting $B$ modes in such data cuts, each of which will have sensitivity reduced by a factor that ranges from $\sqrt{2}$ to a few.

\subsection{Survey requirements and goals - summary}

Although there is some flexibility, there are nevertheless stringent specifications that the survey must meet to reach its main science objectives. These requirements can be summarised as follows.

- The survey must map a large fraction of sky, allowing us to produce foreground-cleaned maps of CMB polarisation over at least $50 \%$ of the sky in a least two (but preferably three or more) different frequency channels in the $100-200 \mathrm{GHz}$ range.

- The survey must be sufficient to unambiguously confirm the inflationary origin of any detected large-scale $B$-mode signal for the targetted value of $r$. For this purpose, both the reionisation bump below $\ell=10$ and the recombination bump up to $\ell \simeq 200$ must be detected, with the ability to see the turnover above $\ell=80$. For $r=0.001$, a foregroundcleaned, delensed CMB $B$-mode map is required to have noise level $\lesssim 3 \mu \mathrm{K}$.arcmin at angular resolution $30^{\prime}$ or better.

- All foregrounds must be mapped at several frequencies, with sufficient angular resolution for cleaning their emission in the CMB channels to below the noise sample variance at all $\ell$ between 2 and 200, and for characterising the level of the residuals. In particular, polarised synchrotron and dust must each be mapped in at least four different frequency channels, with $30^{\prime}$ angular resolution or better in each channel.

- Lensing $B$-mode science requires a survey sensitivity $\lesssim 2.5 \mu \mathrm{K}$.arcmin and CMB angular resolution $\lesssim 8^{\prime}$. For foreground cleaning, the dominating dust foreground emission must be mapped at a matching angular resolution in at least four frequency channels. These requirements also guarantee near-ultimate $E$-mode science. 
- The survey must allow for delensing the $B$ modes down to a residual level of about $3 \mu \mathrm{K}$.arcmin over the $50 \%$ of sky used for CMB science, either using the CIB, which must then be mapped up to $\ell=2000$ or better in several frequency channels above $200 \mathrm{GHz}$, or using small-scale CMB polarisation observations, or (preferably) both.

- The survey must allow for all the necessary mitigation strategies for systematic effects.

- Adequate margins and redundancy strategies must be implemented for fulfilling the above requirements.

To maximise the scientific reach of the mission, we design the mission to also fulfill the following goals.

- For the legacy value of the survey, full-sky maps at all frequencies should be obtained by the space mission.

- For exploiting CMB polarisation to its full potential, $E$ and $B$ modes must be mapped with an angular resolution $\lesssim 4^{\prime}$.

- For complementarity with future ground-based CMB observations, dust emission should be mapped from space with an angular resolution of about $2^{\prime}$.

\section{Mission design}

\subsection{Practical constraints}

The mission concept must be designed so that it can be implemented in the framework of an ESA M-class mission, for a total budget $\lesssim E U R 700$ million for the project (funded mostly by ESA with a EUR 550 million cost cap for an M-class mission, the remainder being funded by European national funding agencies and possible international partners). This is also the typical cost envisaged for future NASA Probe-class missions. The spacecraft must fit in the fairing of the future Ariane 6.2 launcher. The spacecraft mass and size, and the overall programme cost cannot much exceed what has been implemented for Planck, i.e., a total mass of order 2 (metric) tonnes, a 1.5-m class telescope (for which the diffraction limit at $150 \mathrm{GHz}$ is $\simeq 5.6^{\prime}$ ), and a power consumption of order $2 \mathrm{~kW}$. The drastic performance improvement as compared with Planck will primarily come from an increased number of photon-noise-limited detectors (a few thousand instead of a few tens), from an increased number of frequency channels (for better component separation performance in foreground-dominated regions), and from a highly-redundant observational strategy better suited to the measurement of polarisation, with precise control of all systematic effects that could impact the performance of the mission.

\subsection{Orbit}

The choice of an orbit impacts all aspects of the mission: launch requirements; payload and spacecraft geometry; and size of the telecommunications system. As for WMAP and Planck before, CORE will be in orbit around the second Lagrange point (L2) of the Sun-Earth system, to ensure that the Sun, Earth, and Moon are well away from the line of sight at all times. This has been the baseline for EPIC, for previous versions of CORE (COrE, COrE +), and for PRISM. Alternative proposed missions have considered a circular Sun-synchronous low-Earth orbit (LEO), similar to that of $C O B E$, which relaxes the requirements on the 
launcher, as well as on the communications system for telemetry; this was the case for early versions of LiteBIRD and PIXIE.

The main drawback of an LEO is the contamination of the measurement by sidelobe pickup from celestial bodies. The Moon and the Sun each subtend a solid angle of about $700 \mathrm{arcmin}^{2}$, for an emission temperature of approximately $300 \mathrm{~K}$ and $6000 \mathrm{~K}$, respectively, in the frequency range of interest. The target polarisation sensitivity on the same angular scale is about $0.07 \mu \mathrm{K}$. The level of sidelobe rejection towards the Moon must thus be of the order of $4 \times 10^{9}$ or better, while that of the Sun must be of the order of $8 \times 10^{10}$. From an LEO, the Earth subtends a very large fraction of sky (tens of percent). Inhomogeneities in surface temperature and emissivity, modulated in the far sidelobe pickup of the telescope radiation pattern, are therefore a concern for a sensitive CMB space mission.

The impact of this sidelobe stray light is difficult to assess, and demonstrating prior to launch that it does not degrade the performance of the mission would require comprehensive studies (the far-field integrated sidelobe response under normal instrument observing conditions cannot be reliably measured on the ground). It is hence safer to minimise the risk of sidelobe stray light by design, and select the L2 point as a baseline to keep all of the Sun, Earth, and Moon well away from the main beam of the instrument, as well as masked by a number of screens.

A possible orbit around the Sun-Earth L2 point is shown in figure 3. The size of the orbit impacts the required amount of propellant for injection into orbit, and the maximum elongation of the Earth and Moon with respect to the Sun as seen from the spacecraft. Table 3 lists the typical $\Delta v_{\text {orb }}$ required to inject a 2 -tonne spacecraft into orbit for three possible Lissajous orbits around L2. The maximum Earth elongation with respect to the Sun is also listed; this is relevant for the design of the telecommunications system and for sidelobe rejection.

\begin{tabular}{|l|c|c|c|}
\hline Orbit around L2 & $A_{x} \times A_{y}[\mathrm{~km}]$ & $\Delta v_{\text {orb }}$ & $\theta_{\text {Earth }}$ at maximum \\
\hline \hline Large Lissajous & $250,000 \times 673,000$ & $90 \mathrm{~m} \mathrm{~s}^{-1}$ & $24^{\circ}$ \\
Medium Lissajous & $100,000 \times 320,000$ & $270 \mathrm{~m} \mathrm{~s}^{-1}$ & $12^{\circ}$ \\
Small Lissajous & $50,000 \times 160,000$ & $330 \mathrm{~m} \mathrm{~s}^{-1}$ & $6^{\circ}$ \\
\hline
\end{tabular}

Table 3. Typical required $\Delta v_{\text {orb }}$ and maximum Earth elongation for various Lissajous orbits around the Sun-Earth L2 Lagrange point.

\subsection{Observing strategy}

Following the survey requirements, the observing strategy is selected to cover the whole sky in temperature and polarisation, with redundancy of the measurement over many different periods of time. As discussed in section 4.6, the observational plan should be designed for an adequate control of residuals of $I$ into $Q$ and $U$ (temperature-to-polarisation leakage) after inversion of the linear system of observations, and for adequate control of errors in the polarisation angle (mixing of $Q$ and $U$, which generate leakage of $E$ into $B$ ). To that effect, we place the following requirements and preferences on the observing strategy.

1. Each pixel must be observed by the same detector with many different polarisation angles over the course of the mission, preferably evenly spread in $[0,2 \pi]$ for best polarisation sensitivity (Couchot et al. 1999). 
2. Each pixel must be observed with different polarisation angles on relatively short timescales (timescales on which the calibration parameters are not expected to vary significantly).

3. Each pixel must be observed at very different times during the course of the mission.

4. The line of sight must never come close to the direction of the Sun, Earth, or Moon (this specification must be quantified using models of the 2 -dimensional radiation pattern of the telescope).

5. To avoid strong fluctuations of the temperature of the payload, the solar flux absorbed by the spacecraft should be as constant as possible. The cooling chain should preferably provide stable instrument temperature. Any variation of the payload or instrument temperature should be slow compared to the timescale of polarisation measurements.

6. For negligible impact of low-frequency (additive) noise, most of the pixels should be revisited on timescales of the order of the inverse of the knee frequency of the lowfrequency noise (i.e., the frequency at which the power spectrum of low-frequency noise equals that of the white noise). This makes it possible to remove the low-frequency noise using appropriate data-processing techniques (de Gasperis et al. 2005; KurkiSuonio et al. 2009; Revenu et al. 2000; Tristram et al. 2011).

7. Distant pixels must be observed at time-lags smaller that the typical timescale of any instrumental long-term instability, e.g., low-frequency noise, gain drifts, or payload temperature drifts.

8. For minimising the sample variance (and for the legacy value of the observations), the complete sky must be observed, with integration time being evenly spread over the sky.

9. It may be useful to implement a scanning strategy that can concentrate observations in the cleanest regions of the sky during a mission extension, after identification of such regions during the main survey. A mission design compatible with this option is preferred.

The choice to implement an active polarisation modulation system onboard the spacecraft, using a spinning or stepped HWP, is an important element for the definition of the observing strategy. The use of an HWP simplifies the implementation of requirements 1 and 2 (above) by adding the extra flexibility to rotate the polarisation without rotating the whole spacecraft. However, an HWP also has several drawbacks, discussed in section 6.4, that impact the performance and feasibility of the mission. As a baseline, we favour an observing strategy with no HWP. As a consequence, all the requirements above must be fulfilled by scanning only.

The selected strategy impacts the choice between a spin-stabilised or a 3-axis stabilised spacecraft. With spin-stabilisation, the whole spacecraft rotates around a pre-defined axis that is fixed in the frame of the spacecraft. When the telescope line of sight is offset from this spin axis by some angle $\beta$, the line of sight scans the sky along a circle of radius $\beta$. A large value of $\beta$ combined with fast spinning connects distant points on short timescales (requirement 7 above); this was the case for Planck, for which $\beta=85^{\circ}$. On the other extreme, $\beta=0$ results in a sky pixel being observed with all possible orientations on a short timescale (requirement 2); this is the baseline scanning strategy for PIXIE.

To observe the complete sky, the direction of the spin axis must be changed during the mission lifetime. For Planck, this was achieved using small thrusters that corrected the 

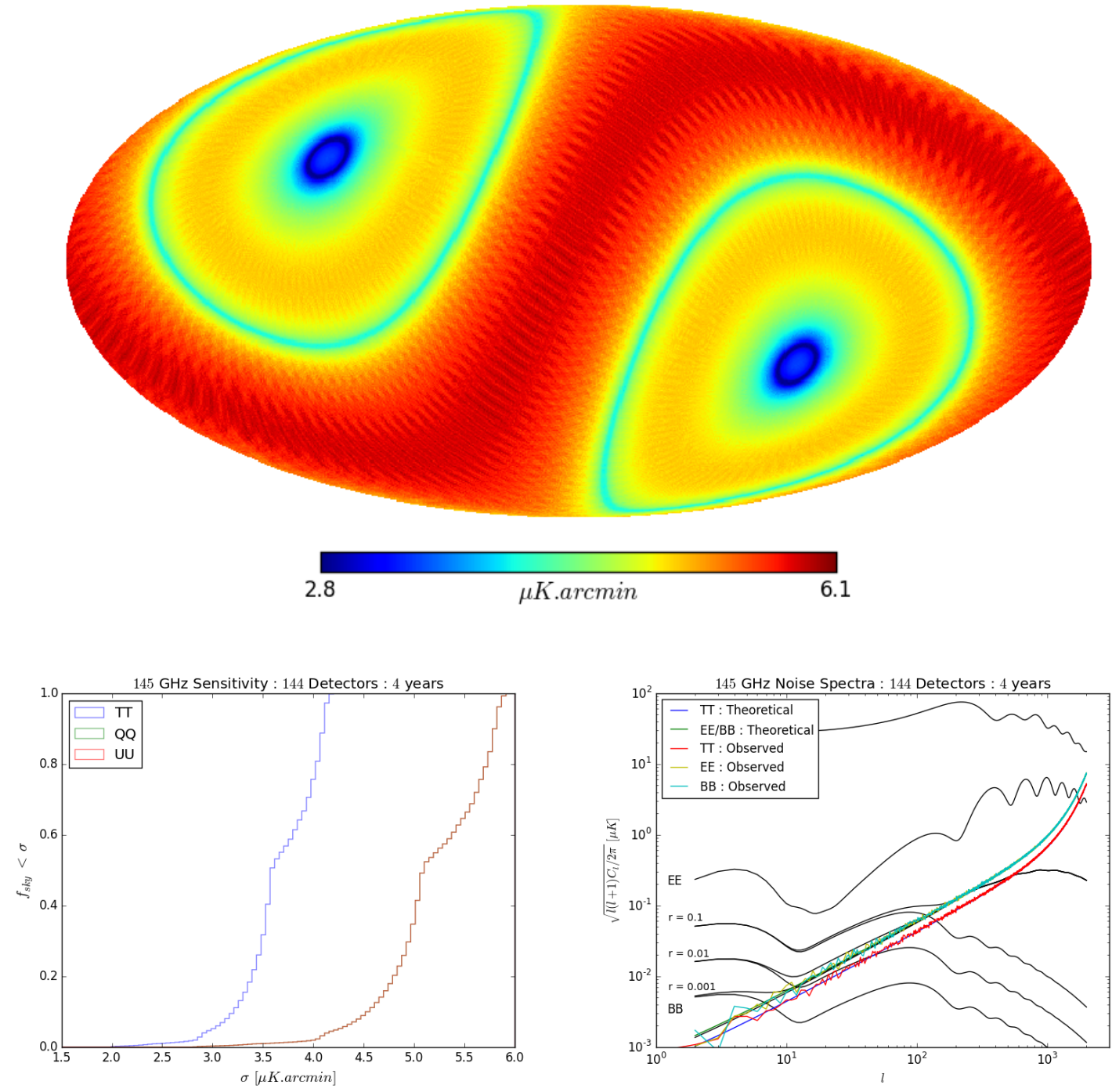

Figure 6. Top: Distribution of the polarisation sensitivity over the sky for the $145 \mathrm{GHz}$ detectors of the CORE baseline design. For this single channel, the sensitivity ranges from about $3 \mu \mathrm{K}$.arcmin in two deep patches located at the ecliptic poles (blue), to about $6 \mu \mathrm{K}$.arcmin near the ecliptic plane, with a median of about $5 \mu \mathrm{K}$.arcmin. Bottom left: Cumulative distribution function of the sensitivity over the sky; most of the pixels are seen with a polarisation sensitivity ranging from 4 to $6 \mu \mathrm{K}$.arcmin (with the $Q$ and $U$ distributions overlapping in the plot). Bottom right: Power spectra of the noise maps for the 145-GHz channel (4 years of observation with 144 detectors distributed in 9 rows of 16), compared to the theoretical estimate from Table 1 for homogeneous coverage.

direction of the spin axis every 40 minutes or so. If motions must be made on smaller timescales, attitude corrections require a significant amount of propellant (see Ref. (Wallis et al. 2017) for a more complete discussion of the corresponding constraints on the mission). With a 3-axis stabilised spacecraft, reaction wheels rotate to keep the satellite in the desired orientation as a function of time. This solution is more flexible, but the scanning speed is limited by the maximum momentum of the reaction wheels, which must compensate for the momentum of the whole spacecraft. 
We select a baseline in which the satellite is spun with a short period $T_{\text {spin }} \simeq 2 \mathrm{~min}$ (frequency $f_{\text {spin }} \simeq 8 \mathrm{mHz}$ ). The spin axis itself will precess with a frequency $f_{\text {prec }}$ (with a longer period $T_{\text {prec }} \simeq 4$ days), around a direction that will be maintained roughly anti-solar (and thus rotate around the Sun with a period of about 1 year to follow the annual motion of the Earth). The spin axis will be offset from the precession axis by an angle $\alpha \simeq 30^{\circ}$, and the optical axis will be offset from the spin axis by an angle $\beta \simeq 65^{\circ}$ (figure 3 ). The resulting temperature and polarisation sensitivity distribution over the sky for a set of 144 detectors at $145 \mathrm{GHz}$, and the resulting noise power spectrum, are shown in figures 6 and 7 .
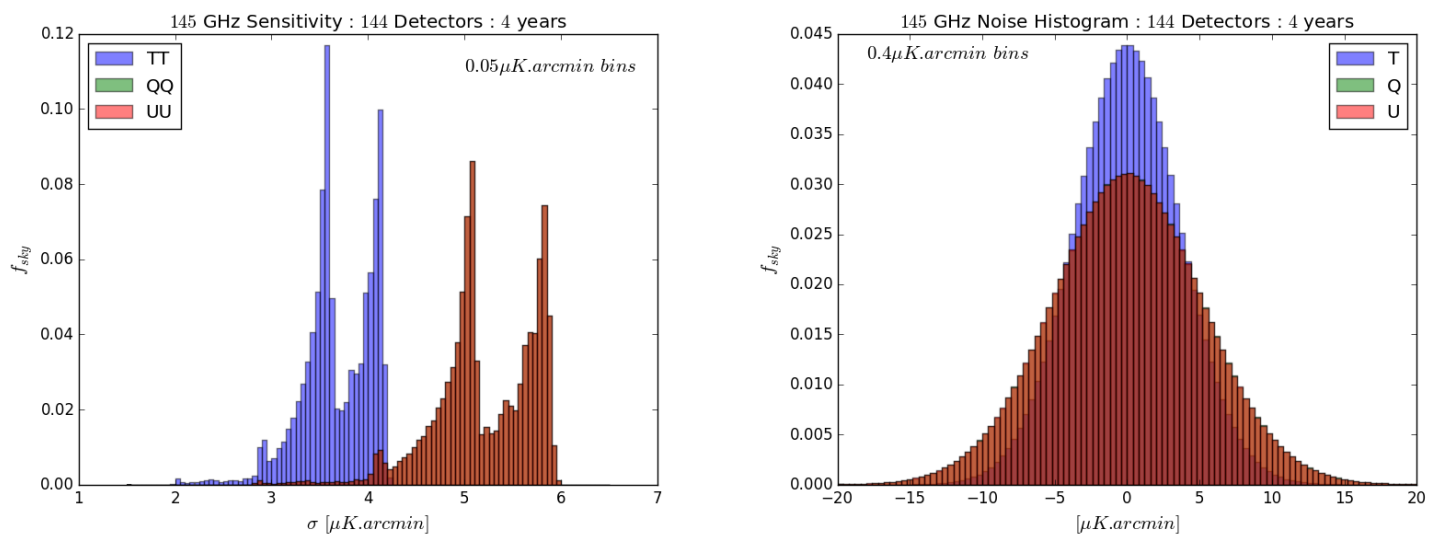

Figure 7. Left: Histogram of noise level for temperature and polarisation, in bins of $\delta \sigma=0.1$; the two peaks of the distribution, lying for polarisation slightly below $5 \mu \mathrm{K}$.arcmin and $6 \mu \mathrm{K}$.arcmin, correspond to the dominating colours in the top panel of figure 6. Right: Histogram of the noise realisation on the map in bins of $0.4 \mu \mathrm{K}$.arcmin. The noise is generated by projection of 4 -year timelines for a patch of 36 detectors each sampled at $85 \mathrm{~Hz}$ onto an $N_{\text {side }}=1024$ HEALPix (Górski et al. 2005) map. The noise values are then rescaled to 144 detectors (four patches of 36 following each other in the scan) and to an equivalent $1 \operatorname{arcmin}^{2}$ pixel size, for easier comparison with the expected noise level. For both panels, the $Q$ and $U$ histograms overlap.

The optimisation of $\alpha$ and $\beta$ is discussed in Ref. (Wallis et al. 2017). In addition to the distribution of observing time over the sky and the distribution of scanning angles for each pixel, these choices impact the design of the payload itself. Further discussion on the tradeoffs that lead to the particular selection of $\alpha$ and $\beta$ values, and of the spin and precession periods, can be found in section 6 and Appendix B.

\subsection{Mission phases and operations}

The main mission phases are listed below.

- Launch and Early Operations phase: The spacecraft will be launched warm, coolers will be turned on shortly after launch. The launcher will place CORE on an orbit towards the Sun-Earth L2 point. Small corrections to the orbit as deemed necessary will take place in the first few days after launch.

- Decontamination phase: The temperature will be allowed to progressively descend to around $170 \mathrm{~K}$, and will be stabilised for a period of about 2 weeks for out-gassing and decontamination of the optical surfaces and of the focal plane. 
- Commissioning and transfer phase: For about 2.5 months, the spacecraft will cruise towards L2. Checks of the basic functionality of the spacecraft and of the payload (commands, AOC, telemetry, and payload basic functionality) will also be performed.

- Calibration and Performance Verification phase: This phase, which will start immediately after injection of CORE on its orbit around L2, and last for about 2 months, will consist of instrument tuning, verification of the sensitivity, initial measurement of instrument key parameters, and initial characterisation of systematic effects.

- Nominal observation phase: For about 4 years, CORE will perform routine scanning of the sky following the selected scanning strategy.

- Extended observation phase: An extension of the mission for deeper integration on selected small patches of sky will be considered if justified by the scientific results obtained from the nominal survey data.

- Decommissioning phase: At the end of operations, the spacecraft will be removed from its nominal orbit around L2, injected into an heliocentric orbit, and passivated.

\subsection{Telemetry}

For Planck, a small Lissajous orbit around L2 was selected to keep the Earth elongation compatible with telecommunication with a fixed, large-beam antenna at the bottom of the spacecraft. With about 30 times more detectors, CORE needs a continuous data rate of about $1.15 \mathrm{Mbits}^{-1}$ (for the baseline configuration). A small (roughly $30 \mathrm{~cm}$ ) steerable antenna operating in the Ka band, communicating with large ground-based antennas currently in use, is compatible with this telemetry requirement.

\section{Payload}

Figure 8 illustrates the conceptual design of the CORE spacecraft. The passively cooled payload module (PLM) is separated from the warm service module (SVM) by a main Sun screen. Throughout the mission, the Sun, Earth, and Moon will always be on the same side of this Sun screen, the PLM being on the other side.

To avoid modulation of the solar flux during the scan, the spacecraft is designed with a general axis of symmetry that coincides with the spin axis. The Sun itself remains, throughout the scanning, on a cone of opening angle $\beta$ with respect to the spacecraft, i.e., always at the same angular distance from the spin axis. This defines a shadow cone on the PLM side, in which all PLM elements must be accommodated to avoid any direct solar illumination during the course of the observations. The Sun screen does not necessarily have to be a flat disc. It is possible, for example, to extend it vertically with a cylinder or a cone to increase the available payload volume.

The payload must be designed to accommodate a telescope that focusses incoming radiation onto a large focal plane. The size of the telescope is driven by the scientific requirements on angular resolution and on the level of sidelobe rejection. The angular diameter of the main beam (between the zeros of the Airy function) of a disc of diameter $D$ is $\theta_{\text {beam }} \simeq 70 \lambda / D$ (in degrees), where $\lambda$ is the wavelength of observation. The FWHM is about half of that for full illumination of the aperture. However, for an aperture illumination with an edge taper of $\lesssim 20 \mathrm{~dB}$, the effective aperture size is close to half of that of the telescope, so that the actual FWHM of the beam is close to the size of the Airy disc. At $150 \mathrm{GHz}(2 \mathrm{~mm})$, a 1-m aperture corresponds to a main beam full width of $\simeq 8.4^{\prime}$, while a 2 -m aperture corresponds to half 


\section{Shadow cone}

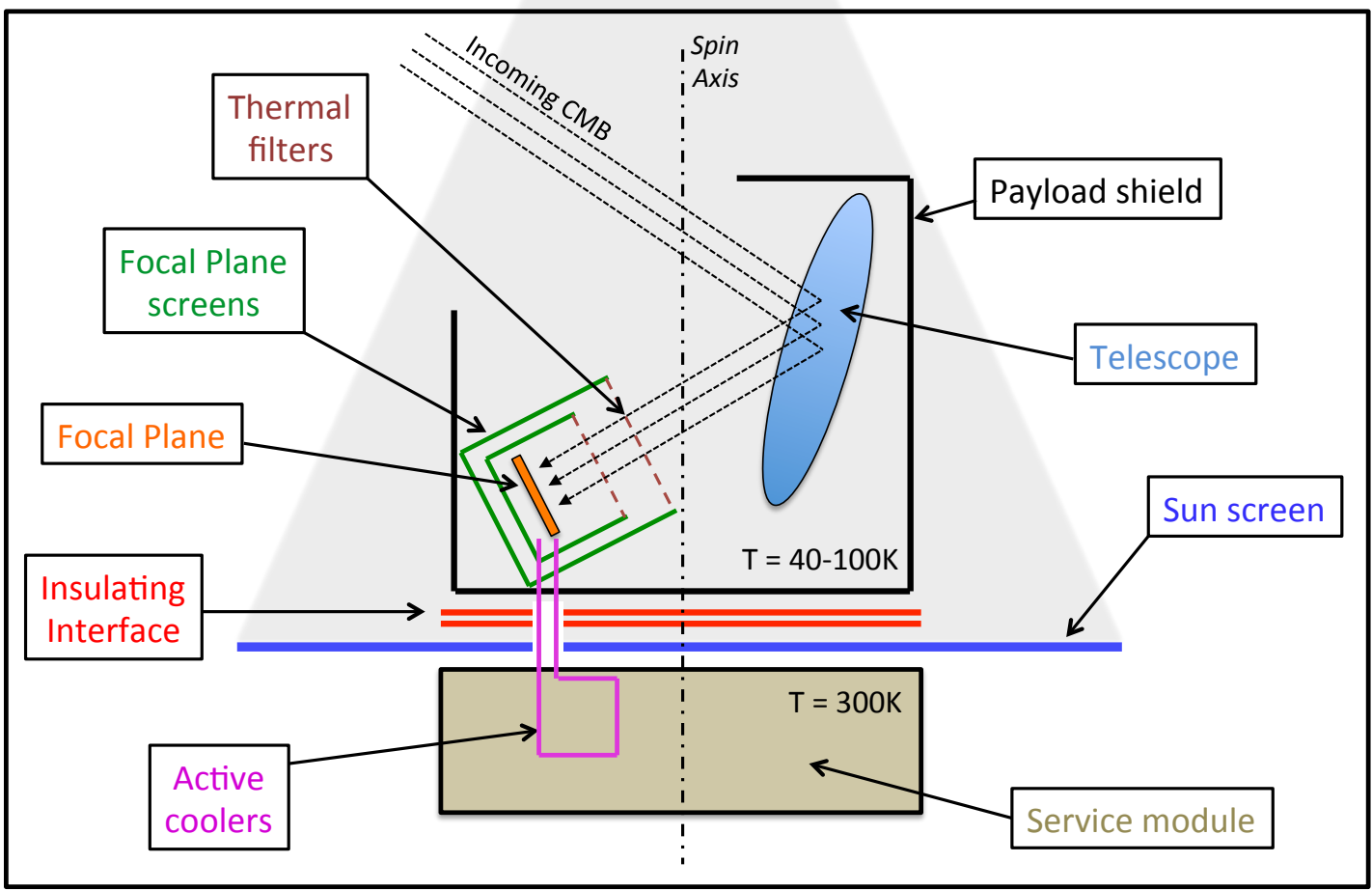

Figure 8. Conceptual sketch of the main elements of the CORE payload; The Sun screen and insulating interface keep the payload in the shade and cold; The telescope focuses the light onto the focal plane; The main payload shield reduces stray light from telescope sidelobes; Active coolers that can be located in the service module, or in a interface element between the service module and the cold payload, cool the focal plane to $100 \mathrm{mK}$, with intermediate stages at $1.7 \mathrm{~K}, 4 \mathrm{~K}$ and $15-20 \mathrm{~K}$.

of that, i.e., $\simeq 4.2^{\prime}$ (actual FWHM beams are slightly different, since they also depend on the exact illumination pattern of the aperture by horns or lenslets in the focal plane, or cold stops if any). To fit in the 4.5-m fairing of an Ariane 6.2 launcher, the focal number of a 1.5 -m telescope should remain $\lesssim 3$ (less for a larger telescope).

The focal plane must accommodate a few thousand background-limited detectors. Hence, the typical diameter of a focal plane observing with thousands of detectors of size $\gtrsim \lambda$ in the $100-200 \mathrm{GHz}$ frequency range is tens of centimetres. Detector coupling using lenslets or horn

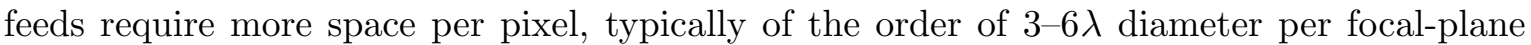
pixel. The larger the focal plane, the more pixels it can accommodate, and hence the better the sensitivity of the instrument. The CORE baseline focal plane is $50 \mathrm{~cm}$ in diameter. For an increased number of detectors, dual-polarisation, multi-frequency detectors, such as those deployed on some ground-based instruments, are an appealing technological solution.

To reach background-limited performance, the detectors must be cryogenically cooled to sub-kelvin temperatures. The space environment makes it possible to passively cool the whole payload itself to a temperature $\lesssim 100 \mathrm{~K}$, to reduce the radiative background from the telescope mirrors and from the payload on the detectors, as well as the thermal load on the 
active cooling stages. Such passive cooling is an advantage, but not a strict necessity - the $\mathrm{CMB}$ can be observed through a warm telescope. As long as the optical coupling of the payload with the detectors is kept below about $1 \%$, the load from a $300-\mathrm{K}$ payload onto the detectors is $\lesssim 3 \mathrm{~K}_{\mathrm{RJ}}$, i.e., comparable to the load from the CMB itself.

A set of reflecting and absorbing shields and baffles prevent stray light from reaching the focal plane. The main payload shield itself has two roles. In addition to being a protection against stray light originating from the sky itself (blocking in particular all spillover radiation around the telescope reflectors), it also contributes to the passive cooling by radiating towards cold space. The same concept was used on Planck, for which the payload temperature was $\simeq 45 \mathrm{~K}$.

\subsection{Instrument design}

The CORE instrument is designed to observe the sky in the frequency range $60-600 \mathrm{GHz}$ with a multi-beam, multi-band polarimeter. As a baseline, the sky emission is collected with a 1.2$\mathrm{m}$ projected aperture telescope that feeds a large focal plane populated with an array of 2100 background-limited detectors. The focal plane is actively cooled to $0.1 \mathrm{~K}$ using a continuumcycle dilution refrigerator. A set of reflecting, low-pass filters reduces the radiative loading reaching the focal plane. Detectors are distributed among 19 frequency bands, each of which have an approximate fractional band-width of $30 \%$. The bands, which monitor foregrounds at the lower and higher end of the spectrum, with the CMB in the middle, are defined by plastic-embedded metal-mesh filters.

Sensitivity to polarisation is obtained by means of plastic-embedded metal grids for all single-polarisation detectors $(\nu>115 \mathrm{GHz})$, and by means of planar ortho-mode transducers for the dual-polarisation detectors $(\nu \leq 115 \mathrm{GHz})$. Radiation is coupled to LEKID resonators by means of embedded-mesh lenslets and short waveguide sections for $\nu \leq 220 \mathrm{GHz}$, and to MKIDs via standard silicon lenslets for $\nu \geq 255 \mathrm{GHz}$. For simplicity, most of the CORE detectors are baselined to be single frequency, single polarisation, but this could be revised in the future, for improved sensitivity. More details can be found in the companion instrument paper (de Bernardis et al. 2017).

\subsection{Telescope}

Two main telescope options have been considered: a Gregorian telescope similar to that of Planck (Tauber et al. 2010b); or a crossed-Dragone design, similar to that considered for EPIC (Bock et al. 2009), LiteBIRD (Ishino et al. 2016; Matsumura et al. 2014), or for future ground-based experiments (Niemack 2016).

The Gregorian telescope has the advantage of compactness for a given aperture. However, the focal surface, neither flat nor telecentric, is not optimally suited to big arrays of detectors. This drawback could be corrected with the use of tertiary optics, e.g., either a refractive lens, or a set of lenses. As lenses are emissive (typical emissivity $\lesssim 10 \%$ ), they must be cooled to cryogenic temperatures, typically below $20 \mathrm{~K}$ to contribute negligible background loading. For a good optical efficiency of the whole system, they must also be covered with anti-reflection coating, which is hard to achieve for a very broad band.

The crossed-Dragone design offers a larger, flat, near-telecentric focal plane, but is more cumbersome, and hence harder to fit into the fairing for a $1.2-\mathrm{m}$ aperture. In addition, the focal plane is near the incoming beam, and is thus exposed to direct illumination from the sky, which is a source of sidelobe contamination. The baseline we consider, shown in figure 9 , solves both problems with a relatively large focal number $(F \simeq 2.5)$ and a flat tertiary mirror, 

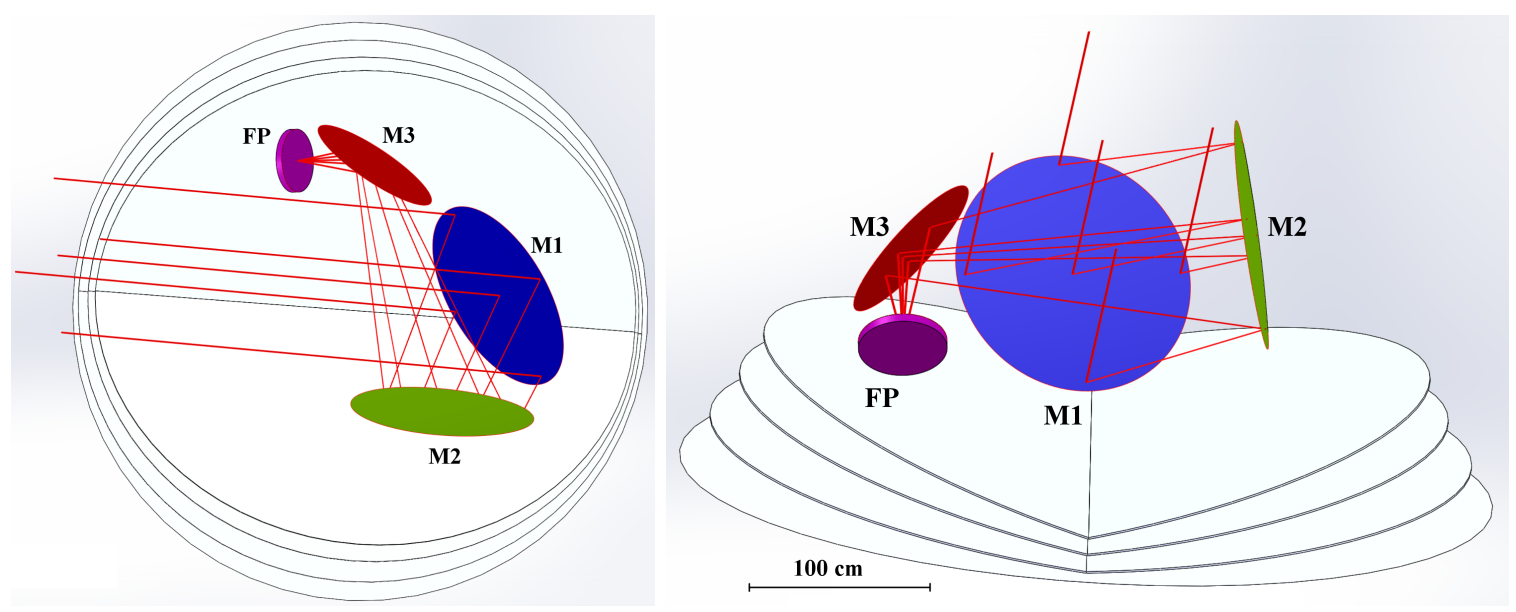

Figure 9. View of the CORE optical system, on top of the V-grooves. Shields and structures are not represented. The primary mirror is shown in blue, the secondary mirror in green, the flat tertiary mirror in red, and the focal-plane array in purple.

which re-locates the focal surface so that it fits in the payload and can be shielded from direct illumination from the sky. Further details of the optics are given in the companion instrument paper (de Bernardis et al. 2017).

\subsection{Shielding against sidelobe stray light}

Sidelobe rejection is essential to avoid contaminating CMB measurements with stray light emission originating from bright sky regions away from the line of sight. Difference maps of observations made with Planck in different surveys highlight the variations in the signal observed in two different orientations. Patterns due to differential integrated sidelobe emission are clearly seen in those difference maps (Planck Collaboration et al. 2014c).

A model of the Planck 2-D radiation pattern identifies the main features responsible for the observed sidelobe pickup as spillover around the edge of the secondary mirror and the primary mirror, and reflections on the sides of the main baffle. The level of these features ranges typically from -75 to $-85 \mathrm{~dB}$, while the majority of the remainder of the radiation pattern is at $-95 \mathrm{~dB}$ or below. The total integrated spillover around the primary or the secondary typically is at the level of $0.1-0.2 \%$ (Tauber et al. 2010b).

While the satellite spins to scan the sky, each of these sidelobe patterns also sweeps the sky with a large elongated "beam," of typical size tens of $\mathrm{deg}^{2}$. When such spillover patterns (which can be though of as elongated structures scanning the sky) cross large structures with brightness of a few $\mathrm{mK}$ amplitude, such as the Galactic ridge, they contribute a signal of the order of a few $\mu \mathrm{K}$ equivalent amplitude brightness, extending over few-degree scales. For comparison, the average noise of CORE integrated over a $10 \mathrm{deg}^{2}$ sky patch is about $0.01 \mu \mathrm{K}$, i.e., 100 to 1000 times smaller.

Since sidelobe patterns are very polarised (diffraction around conductive edges polarise the diffracted light), the sidelobe signal is not reduced much by differencing detectors with orthogonal polarisation sensitivity, nor by a rotating HWP. We must control sidelobes down to a level 100 to 1000 times better than was achieved for Planck. For CORE, this is achieved with the combination of: 

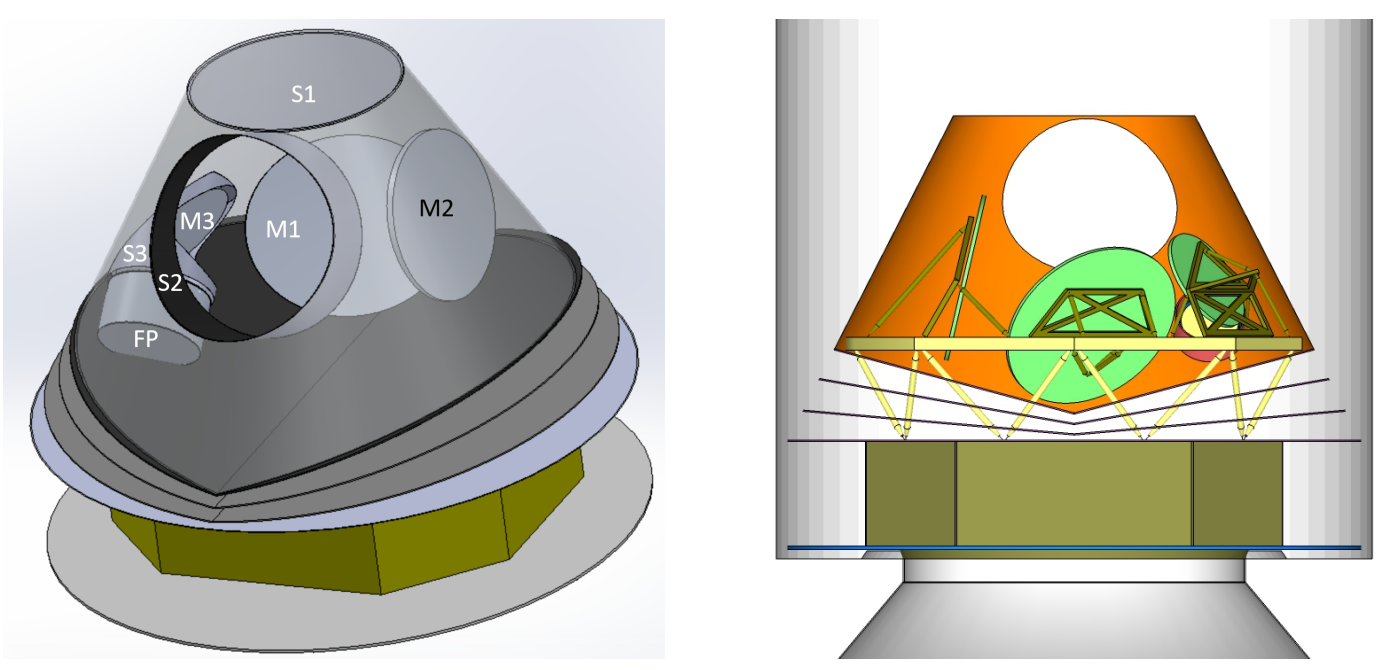

Figure 10. Right: View of the spacecraft, showing the configuration of the three telescope mirrors, M1, M2, and M3, the focal plane, FP, and the various screens. The screens S1, S2, and S3 are designed so that the focal plane is not in direct view of the sky, and hence receives radiation from the sky only through the telescope, or after several reflections on the (mostly absorptive) screens. Left: Side view of the spacecraft in the fairing of an Ariane 6 launcher, illustrating the "book-shaped" geometry of the $\mathrm{V}$-grooves.

- under-illumination of the secondary and primary mirrors, so as reduce direct spillover around telescope reflectors (we assume a typical required edge taper similar to that of Planck, i.e., of the order of $20-25 \mathrm{~dB}$ );

- an approximately $90 \%$ absorptive payload main screen, which reduces the level of stray light by a factor of about 10 at each (partial) reflection;

- a main baffle geometry such that the $10 \%$ reflected power off each absorptive surface is primarily reflected to another absorptive surface, away from the focal plane;

- additional absorptive baffles that avoid any direct view of the sky from the focal-plane array.

This design results in additional rejection of sidelobe pickup by as many orders of magnitude as the number of (partial) reflections that are required for incoming stray light to reach the focal plane, i.e., at least 1 order of magnitude, but likely more. The CORE baffling/shielding design is shown in figure 10 .

In addition to being designed for the minimisation of the sidelobe stray light contamination, CORE is a highly redundant mission. Each 4-detector set observes a connected map of $45 \%$ of sky every 4 days. This provides thousands of independant maps of each sky region, obtained at different times, with different spacecraft orientation, and different detector sets, that can be compared to find signatures of any detectable residual of stray light contamination.

Absorptive screens have the drawback of increased load on the detectors and on the active cooling chain, and thus should preferably be as cold as possible. The other drawback is the impact of temperature fluctuations of the payload on the detected signals. A 1-mK temperature fluctuation of a payload $1 \%$ coupled to the detectors generates a $10-\mu \mathrm{K}$ signal. Such internal stray light emission is usually not correlated to the spacecraft pointing on the 
sky (contrarily to sidelobe stray light), and thus generates spurious signals that are additive (and uncorrelated with the sky emission), rather than multiplicative. They can be removed by processing in a map-making step, along with other low-frequency noise terms; they should nonetheless be kept small by design.

\subsection{Polarisation modulation}

Some CMB polarisation experiments make use of active modulation of the polarisation with a rotating half-wave plate (HWP). Polarisation modulation with an ideal HWP has the advantage of uniformly spreading the angles of polarisation sensitivity in each observed pixel (for optimal polarisation sensitivity), and of mitigating some systematic effects, such as those due to beam asymmetry (when a single detector observing sky pixels with various orientations is used to make polarisation maps). When continuously rotated at a frequency $f_{\mathrm{HWP}}$, it also shifts the frequency of the useful signal to wings of a carrier located at $4 f_{\mathrm{HWP}}$ in the frequency domain, while the signal of a spinning telescope is concentrated in narrow frequencies around multiples of $f_{\text {spin }}$. A fast modulation moves the signal away from the frequency range impacted by any long-term instabilities, such as low-frequency noise gain drifts, and more generally time-evolution of the instrumental response. These desirable properties make the use of HWPs appealing for measuring CMB polarisation. However, there also are several drawbacks to the use of an HWP (in particular in a satellite mission). Perfect modulation is technologically hard to implement, and modulation by an imperfect HWP is likely to generate systematic effects of its own, which might be hard to correct in the data analysis processing.

Whether or not to use an HWP is not a simple question - it must be answered in a difficult trade-off between the advantages, the impact on science, and the risks. The drawbacks of an HWP can be serious, so an HWP should be used only if it clearly helps mitigate critical systematic effects that cannot be avoided by any other means. The CORE baseline mission does not make use of an HWP. We explain why in more detail below.

\subsubsection{Technical complexity}

A rotating HWP in space is a (possibly large) cryogenic moving part on the payload. If continuously rotating on a superconducting magnetic bearing, it is not straightforwardly thermalised, and is also susceptible to being a source of magnetic fields close to the focal plane. Since detectors and their readouts are susceptible to magnetic fields, enhanced magnetic shielding will be required. The angular position (orientation of the principal axes of the birefringent material) must be accurately known at all times to avoid time-dependent $E-B$ mixing. Any such error impacts all focal-plane detectors in a correlated way.

A stepped HWP can be clamped to be thermalised more easily. However, it does not modulate the signal towards wings of $4 f_{\mathrm{HWP}}$ in the frequency domain anymore, and hence does not help with low-frequency noise and long-term instability. It also requires an accurate clamping mechanism to precisely know the HWP orientation each time it is fixed (in a reproducible way).

These technical issues can probably be solved (half-wave plates are operated in many sub-orbital experiments with no obvious show-stopper so far). In space however, they generate instrumental complexity (and hence cost and risk). This must be taken into account in the decision process. 


\subsubsection{Impact on science performance}

Besides the technical challenges, the first major drawback of an HWP is the impact on the scientific capabilities of the mission.

- An HWP reduces the sensitivity of the focal-plane instrument, as compared to the broad-band photon-noise limit, either by reason of extra loading due to radiation emitted by the HWP material and/or (as was the case for the COrE M3 proposal) by constraining the width of the spectral bands. To avoid extra loading, the HWP must be cold, so that the product $\epsilon \times T$ (emissivity times temperature) is a fraction of the sky load (dominated by the $3-\mathrm{K}$ CMB blackbody).

- A single HWP is efficient only in a restricted range of available frequencies of observation, so that covering the full frequency range required for proper monitoring of foreground emission is not guaranteed. As a result, either several instruments covering different frequency ranges must be implemented (with an independent HWP each), or the capability to monitor foreground emission is potentially reduced.

- Used as a first optical element, practical considerations of technical feasibility result in a restriction in the size of the optical aperture, and hence of the angular resolution and of the throughput of the optics (large half-wave plates are challenging). This impacts negatively the scientific reach of the mission, through the ability to de-lens $B$-modes, component separation, and sidelobe rejection, since a smaller aperture has more farsidelobe pickup.

A reflective HWP was proposed as a baseline for the "M3" version of the COrE. The original proposed design used a polarising grid in front of a mirror, a design that allows for a reasonably large aperture (a baseline of $1.2 \mathrm{~m}$ in the COrE proposal) with relatively low emissivity. It also avoided the limited frequency range of operation of current transmissive HWPs. This concept had several appealing properties, its main drawback (besides technological readiness) being a polarisation-modulation efficiency that strongly depended on the frequency of observation. With such a design, optimal polarisation modulation is achieved at frequencies of $(2 N+1) \nu_{0}$, where $\nu_{0}$ is a fundamental frequency set by the distance between the grid and the mirror (and was $15 \mathrm{GHz}$ for $\operatorname{COrE}$ ), and $N$ is an integer. As a result, the experiment could only observe with high efficiency at frequencies centred around 45, 75, 105, 135, 165, $195, \ldots \mathrm{GHz}$, in bands of typical width $15 \mathrm{GHz}$. This constraint generates two difficulties. Firstly, the set of frequencies available for observation cannot be completely optimised for foreground subtraction. The COrE concept had only three channels below $130 \mathrm{GHz}$ to monitor synchrotron emission, which was presumed to be good enough but without much margin for surprises. Secondly, at high frequency, the width of the bands is small, of the order of only $10 \%$ at 135 and $165 \mathrm{GHz}$ (which are the main frequency channels for mapping the CMB). To compensate for the narrow band, many detectors must be deployed, more than 6300 for $C O r E$ to reach a final CMB sensitivity not better than what is achieved with CORE as proposed for "M5," with only 2100 detectors.

A novel type of reflective half-wave plate (R-HWP) was manufactured and tested in recent years, as a result of an ESA funded project, "Large radii half-wave plate development," aimed at the development of this technology for future CMB satellite missions. The new design, based on embedded mesh technology, has high polarisation-modulation efficiency across a $150 \%$ bandwidth at incidence angles up to $45^{\circ}$. The design could be further improved to achieve the $164 \%$ bandwidth required for CORE if necessary. In that case, one could 
consider it either for a payload design similar to that of COrE (M3 version), or for an M5type concept (baseline design presented in the present paper), for replacing the tertiary mirror of the telescope with such an R-HWP. Both options would preserve the angular resolution and sensitivity of the baseline CORE design. However, an embedded mesh HWP is still more emissive than a simple reflecting surface, and hence would negatively impact the sensitivity of the mission, unless it is cooled down to about $10 \mathrm{~K}$ (depending on the exact emissivity). The reflective HWP has the advantage of easier thermalisation, since it has a reflective side that can be thermally conductive.

Transmissive HWPs are an alternative to the reflective concept proposed for COrE. Sapphire HWPs are used on balloons or in ground-based experiments (Hill et al. 2016; Kusaka et al. 2014; The EBEX Collaboration et al. 2017). However, sapphire HWPs are currently limited in size (to $\lesssim 50 \mathrm{~cm}$ ). As an HWP modulates all the incoming polarisation, it is best to use it as a first optical element, so that instrumental polarisation, such as induced by the telescope, is not modulated. This has a consequence on the size of the entrance aperture.

To give a concrete example, let us assume that we select a design with a $30-\mathrm{cm}$ transmissive HWP as the first element in the optical chain. Such an aperture means that the angular resolution would be a factor of approximately 4 times poorer than that of the CORE baseline. The resulting angular resolution in CMB channels (of the order of $30^{\prime}$ at $150 \mathrm{GHz}$ instead of $7.5^{\prime}$ ), is sufficient in principle to search for primordial $B$ modes. However, component separation is compromised by the fact that in the CORE lowest frequency channel $(60 \mathrm{GHz})$, the angular resolution would be about $75^{\prime}$ instead, 2.5 times poorer than at $150 \mathrm{GHz}$. This does not allow for component separation with the full set of frequencies down to the $30^{\prime}$ angular scale. In addition, at $75^{\prime}$ angular resolution, masking strong, polarised, and mostly variable radio sources that contaminate the polarisation maps will leave many missing pixels. Such a small aperture would also have an impact on the sensitivity of the mission, since a smaller telescope necessarily means less throughput and a smaller available focal-plane area for a given acceptable Strehl ratio. For a similar detector technology and implementation design, the loss of sensitivity is directly proportional to the decrease in aperture diameter, i.e., a factor of 4 , for a final sensitivity of $6.8 \mu \mathrm{K}$.arcmin for the full array (instead of 1.7), clearly insufficient to reach the scientific objectives of CORE.

\subsubsection{Mitigation or generation of systematics?}

An HWP mitigates some systematics. A perfect HWP mitigates the impact of beam asymmetry (assuming that the beam does not change with the rotation). It relaxes the need to know the satellite attitude and pointing accurately. It allows for polarisation measurements with single detectors, relaxing the need to know their spectral response very accurately. It also helps with the impact of long-term instabilities that generate low-frequency noise, which are modulated out of the main sky signal frequencies. An HWP, however, does not solve everything; sidelobe signals, for instance, are modulated by the HWP at the same rate as the scientific signal incoming from the main beam of the instrument.

A rotating HWP will also generate systematic effects of its own, which must be dealt with in the analysis of the observational data (Essinger-Hileman et al. 2016; Moncelsi et al. 2014). Chromatic effects on polarisation rotation angles and polarisation efficiency must be accounted for. Also, if detectors do not "illuminate" the HWP homogeneously (i.e., if the full HWP aperture is not homogeneously coupled to the detectors by the downstream optics), which is usually the case, any inhomogenities in the HWP thermal emission will generate spurious signals at the HWP spin frequency and harmonics. To give the rough size, assuming 
illumination inhomogeneities of the order of $10 \%$, and $0.5 \%$ temperature inhomogeneities of a 5 -K HWP with $1 \%$ emissivity, we obtain a spurious signal of the order of $2.5 \mu \mathrm{K}, 3$ orders of magnitude larger than targeted CMB $B$ modes (note that in reality, illumination inhomogeneity is larger than that for edge tapering the aperture against sidelobes, but the tapering is approximately symmetric, and the symmetric part of the illumination does not generate this type of systematic effect).

Even for an achromatic, perfectly thermalised HWP, systematics due to transmission inhomogeneities will be present. Inhomogeneities of the HWP transmission of the order of $0.1 \%$, modulating a $3-\mathrm{K} \mathrm{CMB}$ signal, generate fluctuations of the order of $3 \mathrm{mK}$ across the HWP aperture. Even if the impact of these inhomogeneities averages out by integration over the aperture, a small fraction will subsist, generating spurious signals at the HWP rotation frequency and harmonics. Whether or not this signal can be kept below the noise must be demonstrated with further work, since cancellation by several orders of magnitude is needed.

The impact of many of these imperfections can presumably be addressed in the data analysis procedures. However, an HWP in the payload makes the response and properties of the instrument explicitly time dependent (by design). This time dependence exists not only for the instrument's required polarisation response, but also for many instrumental imperfections. Instead of calibrating these instrumental imperfections for one single stable instrument, with a rotating HWP they would have to be characterised for each polarisation angle (for instance, one sidelobe pattern for each HWP angle). If so, in-flight calibration of instrument properties and data analysis would be significantly more challenging than with a single, stable instrument with no rotating HWP.

Weighing the pros and cons, a design with no HWP seems both to be easier and to allow for improved scientific performance. Hence it is the baseline for CORE. The mitigation of systematic effects without an HWP is further discussed in section 7, as well as in the companion paper on systematic effects (Natoli et al. 2017), in which simulation based analyses show how polarization can be recovered without making use of a HWP.

\subsection{Cooling chain}

The performance of CORE (and more generally of any future CMB space mission) is critically dependent on operating a (potentially large) array of sub-kelvin detectors in space. Such low temperature instruments have already been operated in previous space missions such as Planck (Planck Collaboration et al. 2011), Herschel (Collaudin et al. 2010), and Hitomi (Sneiderman et al. 2016), and are also planned for future missions such as Athena (Branco et al. 2014; Charles et al. 2016). Nonetheless, cryogenic detectors in space are a challenge, and an element of risk for the performance of the mission.

Following the strategy adopted for the Planck satellite, CORE uses the environment of space to achieve the lowest possible payload temperature by passive cooling. Active coolers provide stages at around $15-20 \mathrm{~K}, 4 \mathrm{~K}, 2 \mathrm{~K}$, and $100 \mathrm{mK}$, with shields and thermal filters at each stage that screen the coldest stages from the power radiated by the hotter ones. The baseline active coolers, all European-made, are pulse tubes for the 15-20-K stage, JouleThomson $4-\mathrm{K}$ and $1.7-\mathrm{K}$ coolers, and a continuous ${ }^{3} \mathrm{He}-{ }^{4} \mathrm{He}$ dilution fridge for the sub-kelvin stage.

The development of the active cooling chain depends on the question of the redundancy strategy to safeguard the mission against cooler failures. The cooling chain indeed is a single point failure system, and thus it is in general a good philosophy to implement redundancy here. There is, however, a drawback to this, each cooler that is kept off nonetheless thermally 
connects the cold stages to the hot stages, increasing the conductive load. For Planck, the $20-\mathrm{K}$ sorption cooler was redundant (which turned out to be useful to extend the mission lifetime, after faster than expected aging of the first sorption cooler unit), while the lower temperature stages were not duplicated (Planck Collaboration et al. 2011). For CORE we assume redundant cooling down to $1.7 \mathrm{~K}$ as a baseline, but with only one sub-kelvin cooler. This can be reconsidered at later stages, in a trade-off between integration complexity (and hence cost and schedule risk) versus in-flight failure risk. Details of the cooling chain design can be found in the companion instrument paper (de Bernardis et al. 2017).

\subsection{Mass and power budgets}

The mass of the SVM is estimated to be about $1150 \mathrm{~kg}$, and that of the PLM to be about $390 \mathrm{~kg}$ (telescope reflectors, assuming Silicon Carbide, $100 \mathrm{~kg}$; structures, $120 \mathrm{~kg}$; V-grooves, $90 \mathrm{~kg}$; main payload shield, $40 \mathrm{~kg}$; focal-plane instrument and thermal control, $40 \mathrm{~kg}$ ), for a total dry mass of $1540 \mathrm{~kg}$. The mass of propellant needed for orbit injection and operations is $150 \mathrm{~kg}$ (assuming the use of fly wheels for attitude control). The total wet mass is $1690 \mathrm{~kg}$ (amounting to about 2 tonnes when margins are included).

The required on-board power is about $1700 \mathrm{~W}(2100 \mathrm{~W}$ with margins included), dominated by a cooling chain requirement of $1290 \mathrm{~W}$. The main power consumption is taken by the two pulse tubes ( $450 \mathrm{~W}$ each). Solar panels, for a total effective area of $14.2 \mathrm{~m}^{2}$, populated with the latest generation of triple junction $3 \mathrm{G} 30 \%$ solar cells, are illuminated by the Sun under a constant solar incidence angle of $30^{\circ}$. This provides a worst case end of life electrical power slightly above $2300 \mathrm{~W}$ (based on $190 \mathrm{~W} / \mathrm{m}^{2}$ at normal incidence).

\subsection{Scanning strategy and payload design}

The payload described above is designed for a precession angle $\alpha=30^{\circ}$ between the spin axis and the direction of the Sun, and scanning angle $\beta=65^{\circ}$ between the line of sight and the spin axis (see section 5.3). As discussed in Ref. (Wallis et al. 2017) however, temperatureto-polarisation leakage effects are lowered when the precession angle $\alpha$ is increased while the scanning angle $\beta$ is decreased. This is due to the better distribution of polarisation angles over the sky for a pair of detectors. In particular, all pixels can be seen with all possible orientations only when $\alpha \geq \beta$, and with an appropriate choice of the spinning and precession periods..

The choice of the precession angle $\alpha$ impacts the design of several subsystems of the spacecraft. As $\alpha$ is the incidence angle of solar illumination on the bottom panel of the spacecraft, the power on board for a fixed area of solar panels scales as $\cos \alpha$. The total area that is available is $A=\eta \pi D^{2} / 4$, where $D$ is the diameter of the bottom disc, restricted to be less than the diameter of the fairing, and $\eta$ is the fraction of the area that can effectively be used taking into account the space needed for structural elements and telecommunication antenna(s). Assuming $D \simeq 4.5 \mathrm{~m}$ and $\eta=0.9$, the area available for solar panels is $A \simeq 14 \mathrm{~m}^{2}$. For an on-board power of $2.1 \mathrm{~kW}$, assuming $190 \mathrm{~W} / \mathrm{m}^{2}$ from solar panels at normal incidence, the precession angle is constrained to $\alpha \leq 37.8^{\circ}$.

The precession angle also impacts the geometry of the payload. Increasing $\alpha$ reduces the volume of the shadow cone in which the cold payload must fit, unless one accepts that the Sun illuminate the payload screen, or one changes completely the geometry of the payload.

The spinning period $T_{\text {spin }}$ also impacts several sub-systems of the spacecraft. For a 3 -axis stabilised system, the reaction wheels must compensate the total angular momentum of the spacecraft. Assuming a moment of inertia similar to that of Planck, the total angular 
momentum of the spacecraft spinning at $0.5 \mathrm{RPM}$ is $\simeq 105 \mathrm{Nms}$. As large reaction wheels can store $\simeq 70-100 \mathrm{Nms}$, the spacecraft requires two such reaction wheels, which allows for a maximum spin rate of 0.66-0.95 RPM. Pointing reconstruction accuracy also depends on the spin rate, as star sensors used for attitude reconstruction become less accurate when they scan fast. The combination of reaction wheel dimensioning and attitude reconstruction limits the typical allowed spin rate to $\lesssim 2 \mathrm{RPM}$.

The CORE scan-strategy and payload design result of a compromise between these different constraints.

\section{Controlling systematic effects}

The strategy to control systematics with CORE is based on in-flight calibration and deprojection of intensity leakage from polarisation maps in the data analysis process. Such deprojection requires an accurate model of the instrument, which is not immediately available before launch at the required level of accuracy. A first model of the instrument is obtained from a combination of theoretical modelling and ground-based calibration. This first model comprises estimates of: the beams for each detector (shape and pointing direction with respect to the spacecraft reference frame); polarisation parameters (polarisation efficiency and orientation of the polarisation sensitivity with respect to the spacecraft reference frame); spectral response parameters (models and measurements of the spectral bands); and models of radiation patterns, including $4 \pi$ sidelobe patterns. However, none of these ground-based measurements or models can be expected to match the accuracy that is required to invert the system of eq. (4.3) accurately enough for measuring polarisation $B$ modes with errors dominated by the nominal detector sensitivity. Additional instrumental knowledge must be obtained in flight, either with dedicated measurements during a payload calibration phase, or with the scientific data themselves. We sketch in the next subsections the general strategy to achieve this.

\subsection{Systematic-correction mapmaking}

A linearly polarised detector scanning the sky along a (quasi-circular) path $p(t)$ ideally observes

$$
s(p)=I(p)+\eta\left(Q_{\|}(p) \cos 2 \psi+U_{\|}(p) \sin 2 \psi\right),
$$

where $Q_{\|}$and $U_{\|}$stand for linear polarisation Stokes parameters in the frame where the $x$-axis is along the scan and the $y$-axis perpendicular to it, and $\psi$ is the angle of orientation of the polarimeter with respect to the scanning direction, which is fixed by construction of the payload and by the definition of a fixed spin-axis in the spacecraft frame. Here $I, Q_{\|}$, and $U_{\|}$should be understood as Stokes parameters of the sky emission smoothed with some ideal symmetric beam. The polarisation efficiency, $\eta$, is ideally equal to unity, but can be somewhat lower in practice with no major impact on the measurement as long as $\eta$ is large enough (closer to 1 than to 0 ) and that its value is known. The fact that the scanning is quasi-circular (up to the slow precession of the spin-axis) defines at each pixel along the scanning trajectory a natural frame in which the beams (for intensity and polarisation) and the polarimeter orientation are fixed. If the scanning is at constant angular speed $\Omega_{\text {spin }}$ (which we assume), any time constant of the detectors (or more generally the whole impulse response of the detectors and readout system) can also be included into an effective shape of a scanning beam that does not change with time. 
To first order in polarisation and second order in intensity, systematic effects transform the ideal signal of eq. (7.1) into the following:

$$
\begin{aligned}
s(p) & \simeq I(p)+\eta\left(Q_{\|}(p) \cos 2 \psi+U_{\|}(p) \sin 2 \psi\right) \\
& +a_{\|} \nabla_{\|}^{2} I(p)+a_{\perp} \nabla_{\perp}^{2} I(p)+a_{\times} \nabla_{\perp} \nabla_{\|} I(p) \\
& +b_{\|} \nabla_{\|}\left[I(p)+\eta\left(Q_{\|}(p) \cos 2 \psi+U_{\|}(p) \sin 2 \psi\right)\right] \\
& +b_{\perp} \nabla_{\perp}\left[I(p)+\eta\left(Q_{\|}(p) \cos 2 \psi+U_{\|}(p) \sin 2 \psi\right)\right] \\
& +2 \delta \eta\left[-Q_{\|}(p) \sin 2 \psi+U_{\|}(p) \cos 2 \psi\right] \\
& +\epsilon I(p)+\xi\left[Q_{\|}(p) \cos 2 \psi+U_{\|}(p) \sin 2 \psi\right]
\end{aligned}
$$

where $\nabla_{\|}$and $\nabla_{\perp}$, denote gradients along the scan or perpendicular to the scan, respectively. When several measurements with different orientations (i.e., along different scans) are combined to reconstruct the three Stokes parameters $I, Q$, and $U$, the second line generates a leakage of $I$ into polarisation by reason of beam ellipticity. ${ }^{2}$ The coefficients $a_{\|}, a_{\perp}$, and $a_{\times}$measure the amplitude of each of the terms and depend on the amplitude and direction of the $I$-beam ellipticity with respect to the scanning direction. The third and fourth lines represent the pointing error, and depend on the depointing of the centre of the beam with respect to the nominal direction. This depointing, assumed to be fixed through the duration of the mission, generates in particular a leakage of gradients of $I$ into $E$ and $B$, and of gradients of $E$ into $B$. Note that in eq. (7.2) it is assumed that the displacement is the same for the $I$-beam as it is for the polarisation beams, but this assumption could be relaxed. The third line corresponds to a pointing error along the scan (and could include the impact of an error on the time constant), while the fourth line corresponds to the effect a pointing error across the scan. The fifth line arises from the first-order expansion of the sines and cosines when we replace angle $\psi$ with angle $\psi-\delta$, i.e., describes the impact of a small misalignment of the polarimeter direction in the focal plane. Finally, the sixth line measures the impact of photometric calibration errors and depolarisation due to incorrectly calibrated cross-polarisation leakage.

We assume here that these effects do not vary with time, so that all the parameters, $a_{\|}, a_{\perp}, a_{\times}, b_{\|}, b_{\perp}, \delta, \epsilon$, and $\xi$, are fixed and constant for any given detector (at least for a long-enough period of time to make a map of a substantial fraction of sky). If all of these parameters are known a priori with near-perfect precision (i.e., the instrument is perfectly calibrated), then $b_{\|}, b_{\perp}, \delta$, and $\epsilon$ can be made to vanish (by correcting the pointing solution) and can be ignored. Similarly, the polarisation efficiency correction term (for $\xi \neq 0$ ) can be taken into account immediately in the map-making step by simply changing the value of $\eta$. Only the corrective term from the second line of eq. (7.2), due to beam ellipticity, remains. It is then possible to correct the observations from these remaining systematic leakage effects as follows. First, construct a map of $I$ that ignores them. Use that map to compute the beam-asymmetry terms (the second line of eq. 7.2) and subtract them from the timelines. Then use those timelines to obtain a new map of $I, E$, and $B$ corrected for beam ellipticity. The leakage of $E$ and $B$ into $I$ in the first map-making process for $I$ is a small higher-order correction that can be ignored, although it is possible to iterate the correction if necessary. This method has been investigated and shown in Ref. (Rosset et al. 2007) to perform well on simulations in the framework of preparation for the Planck mission. A new implementation

\footnotetext{
${ }^{2}$ In fact, pointing error also contributes to this term, but if the pointing error is much smaller than the beam size, this is a small correction only.
} 
has been developed specifically for CORE, and demonstrated to reduce the impact of beam asymmetry well below CORE's sensitivity target (de Bernardis et al. 2017; Natoli et al. 2017).

It is theoretically possible to do even better and correct the maps in the case where the calibration is not perfect and the beam shape not exactly known. Instead of computing the correction terms assuming that all of $a_{\|}, a_{\perp}, a_{\times}, b_{\|}, b_{\perp}, \delta, \epsilon$, and $\xi$ are known, we instead calibrate $a_{\|}, a_{\perp}, a_{\times}, b_{\|}, b_{\perp}$ by fitting for the five unknown parameters in a map-making step (i.e., build the least-square map solution for $I, Q, U$, and all of $a_{\|}, a_{\perp}, a_{\times}, b_{\|}, b_{\perp}$ ) assuming that all $\nabla^{2} I(p)$ and $\nabla I(p)$ terms are known to first order from the first iteration of the reconstruction of the intensity map, so that the system to be solved is linearised. We then iterate once again, injecting in the system $Q_{\|}(p)$ and $U_{\|}(p)$ from the map of $E$ to fit for the terms $\epsilon$ and $\xi$ that govern the leakage of $E$ into $B$.

\subsection{Bandpass leakage correction}

The previous paragraph deals with all of the angular response mismatch of a single detector. When mapmaking requires differencing detectors that have different frequency bands, an additional source of intensity-to-polarisation leakage arises. Each detector $i$ observes the integral of the sky emission over a frequency band $h_{i}(\nu)$, so that the intensity detected by each detector can be written as

$$
d_{i}=\int \mathrm{d} \nu h_{i}(\nu)\left[I_{\nu}+Q_{\nu} \cos 2 \psi_{i}+U_{\nu} \sin 2 \psi_{i}\right]
$$

where $I_{\nu}, Q_{\nu}$, and $U_{\nu}$ now are the Stokes parameters of the sky emission brightness as a function of frequency $\nu$, and $h_{i}(\nu)$ is the frequency band of detector $i$.

The total sky brightness arises from the superposition of emission signals from different astrophysical processes. In a given pixel, the total sky intensity is

$$
I_{\nu}=\sum_{c} f_{\nu c} I_{c}
$$

where $f_{\nu c}$ is the spectral emission law of component $c$, and $I_{c}$ is the amplitude of component $c$ at some reference frequency (typically near the centre of the spectral band defined by $h_{i}(\nu)$ ). Similar equations hold for $Q_{\nu}$ and $U_{\nu}$.

In principle, the spectral emission laws for all of the three Stokes parameters $I, Q$, and $U$ can be different for a given component of sky emission, but here we are primarily concerned with the frequency band mismatch for $I$, which is the dominant term. The total signal observed by detector $i$ is

$$
d_{i}=\sum_{c} a_{i c}\left[I_{c}+Q_{c} \cos 2 \psi_{i}+U_{c} \sin 2 \psi_{i}\right]
$$

where

$$
a_{i c}=\int \mathrm{d} \nu h_{i}(\nu) f_{\nu c}
$$

This multi-component model replaces the single component model of eq. (4.3). Calibrating the observations on one given component (e.g., the CMB) amounts to rescaling the observations so that for all $i, a_{i c}=1$ for that particular component $c$. There is, however, no guarantee that all of the coefficients $a_{i c}$ will be the same for all components contributing significantly to the observed emission; hence in the general multi-component case, it is not 
possible to calibrate the data so that all of the $a_{i c}$ coefficients are equal to unity. A set of different detectors measures in each pixel a mixture of components of the form

$$
\boldsymbol{d}=\sum_{c} \mathrm{~A}_{c} \boldsymbol{s}_{c}+\boldsymbol{n}
$$

with, for each component $c$, a component-specific "mixing matrix" for $I, Q$, and $U$ :

$$
\mathrm{A}_{c}=\left(\begin{array}{ccc}
a_{1 c} & a_{1 c} \cos 2 \psi_{1} & a_{1 c} \sin 2 \psi_{1} \\
a_{2 c} & a_{2 c} \cos 2 \psi_{2} & a_{2 c} \sin 2 \psi_{2} \\
\vdots & \vdots & \vdots \\
a_{N c} & a_{N c} \cos 2 \psi_{N} & a_{N c} \sin 2 \psi_{N}
\end{array}\right) .
$$

The consequence is that there is no immediate way to invert the observations to recover the values of $I=\sum I_{c}, Q=\sum Q_{c}$, and $U=\sum U_{c}$ from multi-detector observations. Unless all of the coefficients $a_{i c}$ are equal, any direct inversion assuming a matrix A calibrated on one of the components will inevitably result in a leakage of $I$ into $Q$ and $U$ for the other components, compromising the interpretation of the observed polarisation. The problem is further complicated by the fact that the emission laws of some of the components vary across the sky.

The band-mismatch problem can potentially be severe for detecting low-level primordial $B$ modes. In units of $\mathrm{MJy} \mathrm{sr}^{-1}$, the emission law of synchrotron scales roughly as $\nu^{-1}$, that of thermal dust emission roughly as $\nu^{3.6}$, and that of the CMB as the derivative with respect to temperature of a $2.725-\mathrm{K}$ blackbody. The very different colours of these various emission processess result in differences of a few percent between the various $a_{i c}$ coefficients. For $\mathrm{CO}$ emission, concentrated in thin spectral lines centred at frequencies that are multiples of $\nu \simeq 115 \mathrm{GHz}$ (and nearby frequencies for isotopologues), the exact spectral response may vary significantly between detectors (e.g., by factors of a few).

Similarly to the impact of the angular response, the problem can be solved iteratively as follows. In a first step, maps of intensity are obtained (neglecting the bandpass mismatch) in several frequency bands. These maps are used to obtain maps of intensity for all components in each of the average frequency bands, in a component-separation step. Estimated component maps $\widehat{\boldsymbol{s}}_{c}$ (with vanishing polarisation at this stage) are then plugged in eq. (7.5). Expanding each mixing matrix as $\mathrm{A}_{c}=\mathrm{A}+\delta \mathrm{A}_{c}$, that equation can be recast as

$$
\boldsymbol{d}=\mathrm{A} s+\sum_{c \neq \mathrm{CMB}} \delta \mathrm{A}_{c} \widehat{\boldsymbol{s}}_{c}+\boldsymbol{n} .
$$

This is a linear system in the unknowns $s$ and $\delta \mathrm{A}_{c}$, which can be solved by standard linear inversion.

This method has been implemented on simulations of CORE, demonstrating that the bandpass-mismatch effect can be reduced to a level compatible with the required mission sensitivity with one iteration, provided that the mismatch between the bands is no worse than was the case for Planck (Natoli et al. 2017). Additional technical details and results, as well as a discussion of a second correction method, can be found in Ref. (Banerji et al. 2017).

\section{Options}

\subsection{Descoping options}

$C O R E$ is an ambitious mission. If a drastic descope were deemed necessary, one could consider reducing the ambitions and concentrating on the observations that cannot be obtained by any 
other means and are crucial for achieving the science goals of CORE, either with the mission alone, or in combination with other observations that could be obtained independently (even if not as well as with CORE). Using the name "MiniCORE" to refer to this descoped mission, we would require MiniCORE to provide at least the following capabilities.

- Clean, multi-frequency, full-sky CMB maps at large and medium angular scales, i.e., at all scales where foreground emission and cosmic variance dominate the errors for measuring $E$ modes and lensing $B$ modes when the noise is below $5 \mu \mathrm{K}$.arcmin, i.e., all scales larger than about $10-15^{\prime}$.

- Full-sky maps of high-frequency foregrounds at all useful scales (i.e., down to a few arcminutes), to complement ground-based CMB observations at the same angular scale.

- CIB maps, for delensing the $B$ modes (independently of methods based on CMB polarisation itself).

A downsized version of CORE, with aperture reduced to $80 \mathrm{~cm}, 900$ detectors instead of 2100 , mission duration of 3 years instead of 4 years, and reduced frequency range, would fulfill these requirements. A possible distribution of frequency channels is outlined in Table 4.

\begin{tabular}{|c|c|c|c|c|c|c|c|c|}
\hline $\begin{array}{c}\text { Channel } \\
{[\mathrm{GHz}]}\end{array}$ & $\begin{array}{c}\text { Beam } \\
{[\operatorname{arcmin}]}\end{array}$ & $N_{\text {det }}$ & $\begin{array}{c}\Delta T \\
{[\mu \mathrm{K} . \operatorname{arcmin}]}\end{array}$ & $\begin{array}{c}\Delta P \\
{[\mu \mathrm{K} \cdot \operatorname{arcmin}]}\end{array}$ & $\begin{array}{c}\Delta I \\
{\left[\mu K_{\mathrm{RJ}} \cdot \operatorname{arcmin}\right]}\end{array}$ & $\begin{array}{c}\Delta I \\
{[\mathrm{kJy} / \mathrm{sr} . \operatorname{arcmin}]}\end{array}$ & $\begin{array}{c}\Delta y \times 10^{6} \\
{\left[y_{\mathrm{SZ}} \cdot \operatorname{arcmin}\right]}\end{array}$ & $\begin{array}{c}\mathrm{PS}(5 \sigma) \\
{[\mathrm{mJy}]}\end{array}$ \\
\hline 100 & 16.93 & 40 & 8.4 & 11.8 & 6.51 & 2.00 & -2.0 & 12.7 \\
\hline 115 & 14.81 & 40 & 8.2 & 11.7 & 5.92 & 2.41 & -2.2 & 13.4 \\
\hline 145 & 11.89 & 90 & 5.6 & 7.9 & 3.34 & 2.16 & -2.0 & 9.6 \\
\hline 160 & 10.84 & 90 & 5.8 & 8.1 & 3.09 & 2.43 & -2.6 & 9.9 \\
\hline 175 & 9.96 & 90 & 6.0 & 8.5 & 2.88 & 2.71 & -3.5 & 10.1 \\
\hline 255 & 6.99 & 90 & 9.3 & 13.1 & 2.15 & 4.29 & 5.7 & 11.2 \\
\hline 295 & 6.08 & 40 & 19.6 & 27.6 & 2.96 & 7.91 & 5.7 & 18.0 \\
\hline 340 & 5.3 & 40 & 31.1 & 43.9 & 2.81 & 9.98 & 5.6 & 19.8 \\
\hline 390 & 4.62 & 40 & 55.9 & 79 & 2.75 & 12.85 & 7.1 & 22.3 \\
\hline 450 & 4.00 & 40 & 120.9 & 171 & 2.75 & 17.11 & 11.3 & 25.7 \\
\hline 520 & 3.46 & 40 & 315.2 & 445.8 & 2.79 & 23.18 & 22.4 & 30.0 \\
\hline
\end{tabular}

Table 4. Possible MiniCORE frequency channels. The sensitivity is calculated for a 3-year mission, assuming $\Delta \nu / \nu=30 \%$ bandwidth, $60 \%$ optical efficiency, total noise of twice the expected photon noise from the sky, and the optics of the instrument being cooled to $85 \mathrm{~K}$. This configuration has 900 detectors, about $55 \%$ of which are located in CMB channels between 130 and $220 \mathrm{GHz}$. Those six CMB channels yield an aggregate CMB sensitivity in polarisation of $3.6 \mu \mathrm{K}$.arcmin $(3.2 \mu \mathrm{K}$.arcmin for the full array). Entries for the thermal SZ Comptonisation parameter $\Delta y$ are negative below $217 \mathrm{GHz}$ (negative part of the tSZ spectral signature).

This downsized option still has angular resolution better than $17^{\prime}$ in all channels, in order to observe both bumps of inflationary $B$ modes. With eight channels between 100 and $220 \mathrm{GHz}$ it can check for foreground contamination in CMB maps, but complementary ground-based observations at $90 \mathrm{GHz}$ (and below $40 \mathrm{GHz}$ for monitoring the synchrotron) would be useful. Dust and CIB are mapped between 255 and $600 \mathrm{GHz}$, with angular resolution ranging from 3 to $7^{\prime}$, which is about adequate for complementarity with future ground-based observations for CMB polarisation science. The aggregate CMB sensitivity of $3.2 \mu \mathrm{K}$.arcmin with the full array, slightly less than 2 times worse than the CORE baseline (4 times worse in power), is still good enough for the lensing $B$ modes to be mapped with $\mathrm{S} / \mathrm{N} \simeq 1.5$. The primordial $B$-mode recombination bump is below the noise for $r \lesssim 0.006$. This is not 
optimal, as it fails to clearly satisfy the "margins and redundancy" requirement. While the final sensitivity can be improved a posteriori with an extension of the mission duration, more descoping would not be adequate for addressing the science goals of CORE and guaranteeing scientific breakthroughs - including for the detection of primordial $B$ modes. MiniCORE as defined here can hence be considered as the "minimal" next-generation CMB polarisation space mission.

This downsizing does not require any major redesign of the spacecraft. The smaller telescope and payload would fit in an Ariane 6.2 launcher, without the need of a tertiary mirror (provided the focal plane can still be shielded from stray light). The mass of the focal plane would be drastically reduced, to about $3 \mathrm{~kg}$ instead of $8 \mathrm{~kg}$, allowing for reduced conductive losses and hence more margin on the cooling power. Additional details about the instrumental configuration of this descoped option are available in the companion instrument paper in this series (de Bernardis et al. 2017).

\subsection{Upgrades}

Going in the other direction, CORE could be improved in several ways for better scientific performance. The most straightforward improvement would be to increase the sensitivity by making all detectors dual-polarisation. This improves the noise level in all channels centred at $\nu \geq 115 \mathrm{GHz}$ by a factor $\sqrt{2}$, for a total CMB sensitivity of $1.5 \mu \mathrm{K}$.arcmin using channels from 130 to $220 \mathrm{GHz}(1.3 \mu \mathrm{K}$.arcmin for the full array).

Another simple improvement with little impact on the overall design is to add frequency channels above $600 \mathrm{GHz}$, for better addressing the Galactic and extragalactic science goals. For instance, 96 photon-noise-limited detectors at $1200 \mathrm{GHz}$ would increase the sensitivity to dust and IR sources, at an improved angular resolution of $1^{\prime}$ instead of $2^{\prime}$ (which would also provide improved pointing reconstruction using science data). This would require very limited additional resources (negligible focal-plane area and small increase in telemetry).

Similarly, the use of multi-chroic detectors can potentially improve the sensitivity by increasing the number of detectors by a factor of $2-3$, potentially allowing a final map sensitivity of $1 \mu \mathrm{K}$.arcmin or better. This increased sensitivity would be useful for CMB science, however, only if the foreground emission residuals in CMB maps can be reduced by a matching amount.

Finally, one could consider increasing the telescope size for better angular resolution, and hence improved lensing science and Galactic and extragalactic astrophysics studies. A small aperture increase (e.g., aperture diameter $D \simeq 1.5 \mathrm{~m}$ ) could possibly be achieved simply by optimisation of the proposed geometry. A more significant aperture increase (e.g., $D \simeq 1.8 \mathrm{~m}$ ) would require both a revision of the baseline design, and an increase in the overall size of the payload, which probably could only be considered within a large international collaboration. With the large optical system, the available focal-plane area would also be increased, allowing for more detectors and extra sensitivity.

While they do not drastically improve the performance for inflationary science or for investigations of the cosmological model, the added value of increased angular resolution and sensitivity is substantial for cluster science, extragalactic sources, and for exploiting CIB maps to constrain star formation at distant redshifts. Some of the companion science papers to this one investigate the added value of such upgrades. 


\begin{tabular}{|c|c|c|c|c|c|c|c|c|c|c|}
\hline Mission & Year & $N_{\text {det }}$ & $\begin{array}{c}\Delta P \\
{[\mu \mathrm{K} \cdot \operatorname{arcmin}]}\end{array}$ & Aperture & $\begin{array}{c}\text { Beam } \\
\text { size }\end{array}$ & $N_{\nu}$ & $\begin{array}{c}\nu \text { range } \\
{[\mathrm{GHz}]}\end{array}$ & $N_{\text {rec. }}$ & $t_{\text {map }}$ & HWP \\
\hline EPIC-LC (NTD) & 2007 & 830 & 3.0 & $30 \mathrm{~cm}$ & $155^{\prime}-16^{\prime}$ & 7 & $30-300$ & 6 & months & yes \\
\hline LiteBIRD & 2010 & 2622 & 2.5 & 20 and $40 \mathrm{~cm}$ & $69^{\prime}-17^{\prime}$ & $6 \rightarrow 15$ & $40-400$ & 2 & months & yes \\
\hline EPIC-IM (4K) & 2009 & 11094 & $\overline{0.9}$ & $1.4 \mathrm{~m}$ & $28^{\prime}-1^{\prime}$ & 9 & $30-850$ & $\overline{c 1}$ & months & no \\
\hline EPIC-IM (30K) & 2009 & 2022 & 2.3 & $1.4 \mathrm{~m}$ & $28^{\prime}-1^{\prime}$ & 9 & $30-850$ & 1 & months & no \\
\hline CORE (M5) & 2016 & 2100 & 1.7 & $1.2 \mathrm{~m}$ & $18^{\prime}-2^{\prime}$ & 19 & $60-600$ & 1 & few days & no \\
\hline EPIC-CS & 2007 & 1520 & 1.8 & $3.0 \mathrm{~m}$ & $15^{\prime}-1^{\prime}$ & 8 & $30-500$ & 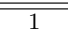 & months & $\overline{\mathrm{TBD}}$ \\
\hline PIXIE & 2010 & 4 & 4.2 & $55 \mathrm{~cm}$ & $2.6^{\circ}$ tophat & 400 & $30-6000$ & 2 & months & no \\
\hline PRISM (imager) & 2013 & 7600 & 1.1 & $3.5 \mathrm{~m}$ & $17^{\prime}-5^{\prime \prime}$ & $32+300$ & $30-6000$ & 1 & few days & no \\
\hline PRISM (spectro) & 2013 & a few & 2.9 & $50 \mathrm{~cm}$ & $1.4^{\circ}$ & 400 & $30-6000$ & 2 & months & no \\
\hline
\end{tabular}

Table 5. Main characteristics of proposed CMB space missions. Columns are, from left to right: mission name (with possible options); year of initial conceptual design; number of detectors; aggregated CMB sensitivity from all channels; aperture size; beam size; number of frequency channels; frequency range; number of receivers (optical systems with a focal plane); typical time required to observe, with a single 4-detector set, a sizeable, well sampled map (e.g., tens of percent of sky, connected, with no holes); and whether or not the mission uses a rotating HWP. The three main vertical sections identify missions focused on large-scale CMB polarisation (category 1, top), missions targeting most of CMB polarisation science (category 2, middle), and missions specifically designed to also address science objectives beyond CMB polarisation (category 3, bottom). In each category, missions are ordered according to the year of design. Entries for LiteBIRD correspond to a recent version with 3 years of observation, in which the initial number of six frequency bands in a single receiver evolved to a new baseline of 15 bands with two receivers. The PIXIE sensitivity assumes a polarisation sensitivity of $70 \mathrm{nK}$ for the full instrument (Kogut et al. 2011). PRISM has two independent instruments, specified on two different rows, but both are part of the proposed baseline mission; the PRISM imager has 32 broad-band channels, and also a narrow-band spectrometer (with $R=\Delta \nu / \nu \simeq 100$, for about 300 narrow spectral bands).

\section{Discussion}

We now turn to a discussion of our choices for CORE and put those choices in the context of other CMB polarisation space missions. Table 5 gives the main characteristics of space missions that have been proposed or studied since 2007. The Table has three parts: (i) missions with telescope aperture $\simeq 30 \mathrm{~cm}$, with relatively coarse angular resolution and noise level $\gtrsim 2 \mu \mathrm{K}$.arcmin, targetting large scale CMB polarisation only; (ii) missions with a telescope size in the 1.2-1.5 m range, with angular resolution of a few arcminutes and a noise level in the 1-2 $\mu \mathrm{K}$.armin range typically, designed for comprehensive CMB polarisation science; (iii) missions specifically designed for addressing a scientific program that extends beyond CMB polarisation, with either high angular resolution, or including CMB spectroscopy as a main science goal. Among the various options, CORE is the right choice for an ESA M-class mission for the following reasons:

- It has the combination of resolution and low noise to give $\sigma_{r}=0.0004$, thus clearly distinguishing between inflationary models with $r \ll 0.001$ or $r=0.003$, avoiding in particular the risk of a possible ambiguous hint of $r=0.002 \pm 0.001$, which would neither be a clear detection, nor rule-out a Starobinsky-type inflationary model for which $r \simeq 0.003$. We designed CORE with this capability because current theory gives strong motivation for such inflationary models, and we believe that to be relevant in the 2020's any space mission must be able to provide this discrimination; Delensing 
capability is essential to reach this level of sensitivity: with lensing reconstructed by CORE, one can reduce the lensing B-mode power by $70 \%$, leading to an improvement of a factor of 2.5 in the error on the amplitude of primordial gravitational waves (Challinor et al. 2017).

- It has the necessary number of frequency bands, with noise per band sufficient to measure sources of galactic emission. Any mission that aspires to measure primordial $B$ modes at $\ell<20$ must contend with foreground levels that are orders of magnitude stronger than the $B$ mode, and must contend with yet unknown potential foreground complexities. Some of the early proposed polarisation missions do not have sufficient foreground determination capabilities;

- A mission that targets only $r$ may have a null-result as its main science output. While setting limits on $r$ has important consequences for the physics at ultra-high energies, the resolution of the mission strengthens the CORE constraints on the physics of the inflation, even if $r$ is not detected, through high fidelity measurements of $n_{\mathrm{s}}$, of the scaledependent running of $n_{\mathrm{s}}$, and of non-Gaussianity. This requires the measurement of the E-mode power spectrum over a broad range of $\ell$ 's. We also believe a next generation CMB space mission should provide a broader range of cosmological and astrophysical results, serve a broader community of astrophysicists, and give a legacy dataset to be mined for more than just inflationary physics. We designed CORE to provide cosmic variance limited observation of the $E$ modes up to $\ell \simeq 2500$ and of the lensing $B$ modes up to $\ell \simeq 1000$, enabling investigation of possible extensions with parameters describing curvature, neutrino physics, extra light relics, primordial helium abundance, dark matter annihilation, recombination physics, variation of fundamental constants, dark energy, modified gravity, reionisation, cosmic birefringence. The ground-breaking post-CORE overall reduction of the allowed parameter space will be as much as $\sim 10^{7}$ as compared to Planck 2015, and $10^{5}$ with respect to Planck $2015+$ future BAO measurements (Di Valentino et al. 2016). The angular resolution of CORE is optimised to probe a broad range of science goals while fitting within an ESA M-class mission budget.

- We designed the scan strategy of CORE to give strong discrimination of polarimetric systematic errors. We considered an alternative approach to mitigate low frequency noise and polarimetric systematic effects - the use of a continuously rotating halfwave plate - a technical risk that (i) would therefore increase costs, and (ii) is not necessary given the mitigation provided by the scan strategy. Experience with suborbital instruments suggests that a half-wave plate should be the first element in the optical path. A half-wave plate, whether rotated continuously or in steps, with an entrance aperture diameter larger than $0.5 \mathrm{~m}$ and compatible with the broad frequency coverage required for foreground cleaning is a technical challenge with consequences on costs and schedule.

\subsection{Complementarity with sub-orbital experiments}

Past experience with CMB temperature anisotropies shows that precision CMB science requires a space mission when the dominating sources of error are foreground contamination, cosmic variance, and systematic effects, rather than raw CMB sensitivity. 
The same will be true for polarisation. While the noise limit for $E$-mode and lensing $B$-mode detection was first overcome by deploying increasing numbers of detectors observing from the South Pole and the Atacama Plateau (not forgetting circumpolar balloon flights), a comprehensive, precise and accurate cosmological exploitation of CMB polarisation (including $E$ modes and lensing $B$ modes), cannot be made without a space mission.

This, however, does not preclude exploiting the best ground-space complementarity. A diffraction limit of $2^{\prime}$ requires a $4.2-\mathrm{m}$ telescope at $150 \mathrm{GHz}(6.3-\mathrm{m}$ at $100 \mathrm{GHz})$. While such telescopes can be deployed on the ground, they cannot be envisaged in orbit within the budget of an M-class mission. As demonstrated with SPT and ACT, even for mapping temperature, such large telescopes perform well for measurements at $\ell \gtrsim 500$. They are a perfect complement to a space mission that observes up to $\ell=1000$ (a $12^{\prime}$ beam) in the same frequency range, providing a good overlap in sensitivity for cross-calibration of gains and beams in the $500 \leq \ell \leq 1000$ angular scale range.

The combination of a spectrometer mission such as PIXIE, an imager such as CORE, and a high resolution ground-based observatory would be very powerful for the best observation and scientific exploitation of the CMB in the next decade.

\section{Conclusion}

Cosmological observations support a concordance inflationary $\Lambda \mathrm{CDM}$ cosmological scenario, in which seeds for density perturbations are generated in the very early Universe during a phase of cosmic inflation, by stretching to macroscopic scales quantum fluctuations of the spacetime metric. These perturbations then evolve in the primordial plasma until baryons decouple from radiation, releasing the $\mathrm{CMB}$, and become free to collapse under the force of gravitation to generate the large-scale structures observed in the present Universe. But fundamental questions still remain: did cosmic inflation really happen, and if so what is the physics that drives it? What are the dark matter and dark energy required by this scenario, which appear to represent $96 \%$ of the total energy density in the Universe? Is there something essential still missing in our understanding of our cosmos?

The CMB is a crucial tool for further investigating this global picture. Three space missions have already scrutinised the CMB to exploit the scientific information encoded in its tiny fluctuations of intensity and polarisation, largely contributing to the adoption of the standard $\Lambda \mathrm{CDM}$ model. However, only temperature anisotropies have been mapped with good signal-to-noise ratio over most of the sky and for most of the useful angular scales. Much can still be learnt from detailed observations of CMB polarisation on all scales larger than about two arcminutes: polarisation $E$ modes are yet a largely unexploited probe of the cosmological model; lensing $B$ modes offer the opportunity to map all the dark matter structures between the last scattering surface at $z \simeq 1080$ and present-day observers, to further understand how it clusters and how it interacts; the detection of primordial $B$ modes on scales larger than about thirty arcminutes is essential to confirm the inflationary scenario and obtain clues about the physics at work in the early Universe, on grand unification energy scales $10^{12}$ times higher that those probed by the largest human-made particle accelerator to date. These new observations of CMB polarisation still have the potential to revolutionise our understanding of our Universe. Their optimal exploitation is a must.

Ideally, CMB polarisation should be observed accurately over the full sky, at all scales down to about $2^{\prime}$, and with a sensitivity of the order of a fraction of a $\mu \mathrm{K}$.arcmin. This is a challenging task. While such a sensitivity can theoretically be reached observing continuously 
for a few years from the ground with several hundred thousand detectors, or from space with several thousand detectors, controlling foreground contamination or systematic effects with a matching level of accuracy is the major challenge to overcome in order to reach the science goals of a future CMB polarisation survey. Space-borne observations of the CMB offer unmatched precision and accuracy when astrophysical foreground emission, cosmic variance, or instrumental systematic effects are the dominant sources of error in the interpretation of CMB observations. This is the case for all angular scales down to about 10 arcminutes; smaller scales, which require telescopes of size several metres, are best observed from groundbased observatories.

The most effective scientific exploitation of CMB polarisation must hence make use of the complementarity between the ground and space. The space mission must map the polarised sky in more than 10 frequency channels with an angular resolution sufficient to cover the range of scales where foregrounds or cosmic variance dominate the errors in CMB maps, i.e., all scales $\gtrsim 10^{\prime}$. Such a space survey can be complemented with ground-based observations to extend the angular resolution down to $2^{\prime}$ in a few atmospheric windows; such information is hard to gather from space by reason of the size of the telescope required to reach this angular resolution at those frequencies. A space mission is, however, ideally suited to map the high-frequency foreground emission (i.e., dust and CIB) above $300 \mathrm{GHz}$, with a matching angular resolution of the order of $2^{\prime}$. This can be achieved with a metre-class telescope in space, while the atmosphere precludes a wide and sensitive survey from the ground at those frequencies. Dust must be mapped to accurately subtract its contribution to CMB $E$ and $B$ modes and to characterise any possible residuals that could bias the extraction of those signals. The CIB must be mapped as a useful tracer of cosmic structure, which is essential to disentangle primordial $B$ modes from lensing.

$C O R E$ reaches the sensitivity and angular resolution requirements of such a future space mission, across a frequency range that extends from 60 to $600 \mathrm{GHz}$, with an array of 2100 cryogenically-cooled detectors at the focus of a 1.2-m aperture telescope. The sky is mapped in 19 frequency channels with angular resolution ranging from 2 to $18^{\prime}$, for an aggregate CMB survey sensitivity of $1.7 \mu \mathrm{K}$.arcmin in polarisation after 4 years of continuous observations. The observing strategy is such that $45 \%$ of the sky is mapped every 4 days, allowing for many cross-checks of the measurement over the course of the mission, essential to control systematic effects. Additionally, the broad frequency coverage, with six independent channels between 130 and $220 \mathrm{GHz}$, and $\mathrm{CMB}$ polarisation sensitivity of order $5 \mu \mathrm{K}$.arcmin each, allows for cross-checking to ensure that the measured CMB spectra are not contaminated by residual foregrounds after subtraction of a model of polarised astrophysical emission. This built-in redundancy is essential to ascertain the accuracy of the observed CMB, and avoid any false interpretation of the observed sky emission.

CORE is designed to minimise systematic effects that could potentially be generated by thermal instability or side lobe pickup of strong astrophysical emission: The spacecraft and the scan-strategy are designed in such a way that the solar power absorbed remains constant throughout the scientific observations. Sun, Earth and Moon are kept well away from the line of sight at all times, and masked by absorptive screens that avoid illumination of the focal plane, either directly or by reflection. With no moving part in the optical path of the instrument, and with continuous observations in a very stable configuration, CORE is designed for a maximally stable instrumental response, allowing to calibrate the instrument in flight with an accuracy matching the stringent requirements of the measurement. This allows for correcting potential systematic effects in a data-processing step that jointly measures the 
sky emission and relevant parameters of the instrumental response.

Should descoping be deemed necessary for technological or programmatic reasons, it would be possible to reduce the aperture size to $80 \mathrm{~cm}$ instead of $120 \mathrm{~cm}$, divide the number of detectors by 2, and drop the lowest frequency channels below $100 \mathrm{GHz}$ (which are the most challenging from space by reason of required volume and mass). This would increase the dependence of the mission upon high-quality measurements from the ground in all atmospheric windows, but would preserve most of the essential CMB polarisation science. Alternatively, the quality of the survey could be improved with dual-polarisation and/or multichroic detector technology, as well as with a somewhat increased telescope aperture.

Although optimised for accurate CMB polarisation observations, CORE also has the potential to tackle additional science goals. Its sensitivity to foreground polarisation will help understand the role of magnetic fields in the structuring the interstellar medium and in Galactic star formation. By detecting tens of thousands of galaxy clusters (and hundreds of thousands in combination with a high-resolution ground-based CMB survey), it will provide a means to constrain the nature of dark matter and dark energy or to check for modified gravity, independently of the primary CMB or of any other probe. By observing distant, strongly lensed dusty galaxies and protoclusters of galaxies, it will help us to understand the history of cosmic star formation and the role of baryons in the formation of matter structures. Finally, the legacy value of the 19 accurate intensity and polarisation maps it will deliver to the scientific community will make the CORE survey a lasting resource for many additional astrophysical and cosmological investigations, the impact of which cannot yet be foreseen.

\section{Acknowledgments}

The CORE collaboration thanks CNES, Thales Alenia Space, and Air Liquide Advanced Technologies for advice and technical support during the preparation of the CORE proposal. We also thank the ESA CDF team for the CMB Polarisation CDF study performed in March 2016, the results of which were extensively used to define the mission concept presented in this paper. J.G.N. acknowledges financial support from the Spanish MINECO for a Ramon y Cajal fellowship (RYC-2013-13256) and the I+D 2015 project AYA2015-65887-P (MINECO/FEDER). CJM is supported by an FCT Research Professorship, contract reference IF/00064/2012, funded by FCT/MCTES (Portugal) and POPH/FSE. F.J.C., R.F.-C., E.M.-G. and P.V. acknowledge support from the Spanish Ministerio de Economa y Competitividad project ESP2015-70646-C2-1-R (cofinanced with EU FEDER funds), ConsoliderIngenio 2010 project CSD2010-00064 and from the CSIC "Proyecto Intramural Especial" project 201550E091. FA is supported by the National Taiwan University (NTU) under Project No. 103R4000 and by the NTU Leung Center for Cosmology and Particle Astrophysics (LeCosPA) under Project No. FI121. BFR acknowledges support from the National Science Centre, Poland, under grant 2014/13/B/ST9/00845. 


\section{A Impact of atmosphere on ground-based CMB observations}

\section{A.1 Atmosphere and detector sensitivity}

Space-borne detectors benefit from a potentially very cold and quiet environment, allowing for the sensitivity of broad-band detectors below $200 \mathrm{GHz}$ to be limited by CMB photon noise. From the ground, typical atmospheric emissivity in atmospheric windows is at the level of a few percent, depending on the frequency and the amount of precipitable water vapour. This extra radiation generates additional loading on the detectors, at the level of a few percent of the atmospheric temperature of $230-290 \mathrm{~K}$ (i.e., 10-20 K of background), rapidly increasing at frequencies above $200 \mathrm{GHz}$. The actual loading observed with the BICEP2 instrument is $22 \mathrm{~K}_{\mathrm{RJ}}$ (BICEP2 Collaboration et al. 2014), partly due to the atmosphere, the rest being due to the instrument itself (forebaffle, window and filters).
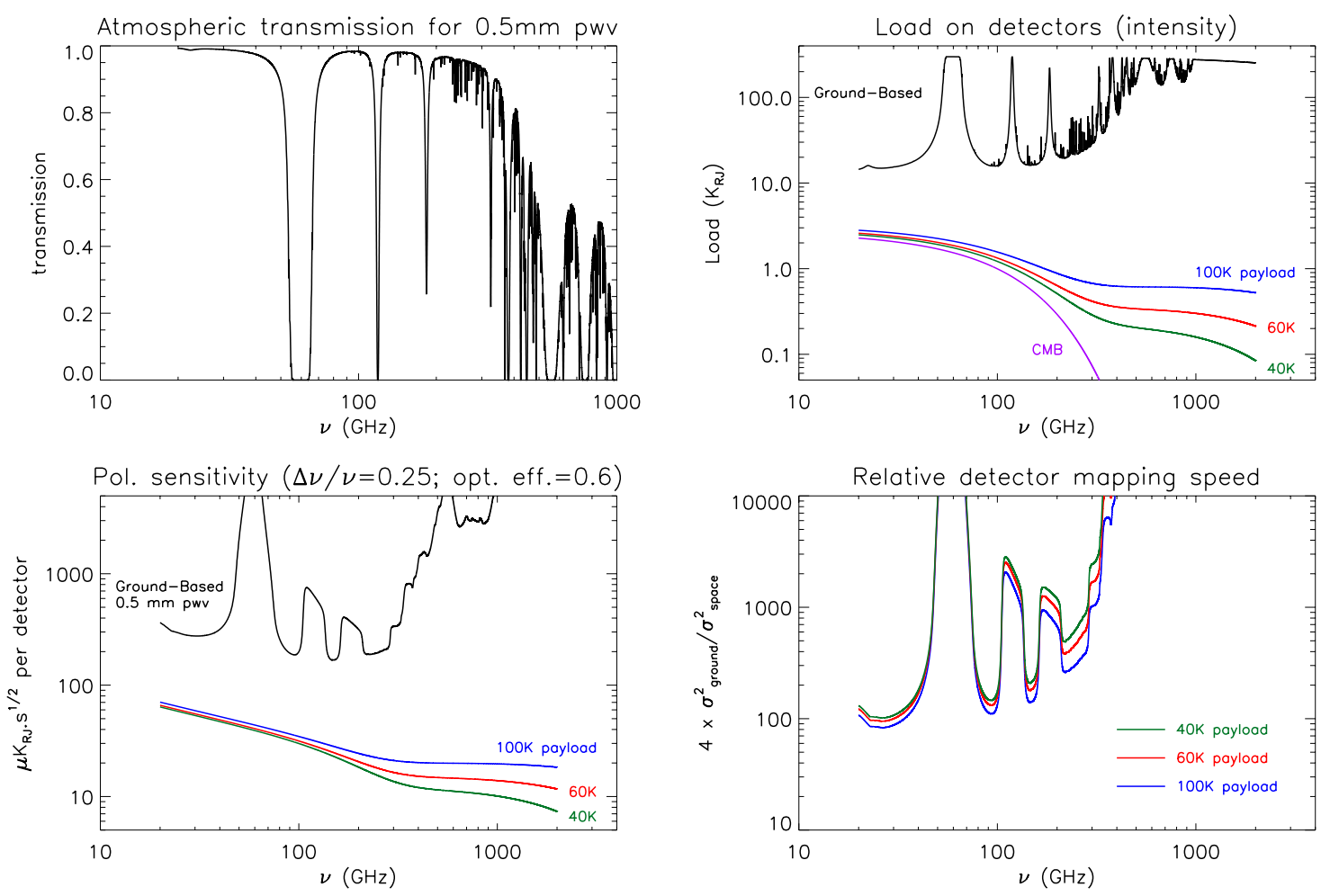

Figure 11. Top left: Typical atmospheric transmission from the Atacama plateau at $60^{\circ}$ elevation, for an average of half a millimetre of integrated precipitable water vapour. Top right: Load on a detector for a ground-based instrument (black) and for a space-borne instrument with various payload temperatures. In the ground-based case, we assume $3 \%$ total emissivity for the optics (telescope reflectors and entrance window), and assume that all the environment is at $290 \mathrm{~K}$, while for the space mission we assume a telescope emissivity similar to that of Planck, and $0.5 \%$ stray light from a black payload. Bottom left: Corresponding detector sensitivity (noise level of a single polarised detector calibrated in intensity) as a function of band central frequency; we assume square bands with $\Delta \nu / \nu=0.25$, an optical efficiency of $60 \%$, and consider single-moded detectors with a throughput of $\lambda^{2}$. Bottom right: Relative mapping speed of one single space-borne versus one single ground-based detector with the same assumptions, also considering an observing efficiency of $25 \%$ from the ground as compared to space.

Figure 11 compares the background load and noise level for a detector in the focal 
plane of a $40-\mathrm{K}, 60-\mathrm{K}$, or $100-\mathrm{K}$ space-borne telescope with emissivity similar to that of Planck, with those of a ground-based detector with $3 \%$ emissive optics (total emissivity for the telescope and the cryostat window if any), and $0.5 \mathrm{~mm}$ of precipitable water vapour, observing at $60^{\circ}$ elevation from the Atacama plateau. The emissivity of the space telescope is modelled following the measured performance of the Planck reflectors (Tauber et al. 2010b). We assume square frequency bands with $25 \%$ bandwidth, $60 \%$ optical efficiency, and assume that each detector integrates incoming radiation over a throughput of $\lambda^{2}$, where $\lambda$ is the central wavelength of the band. We also assume that the total noise is $\sqrt{2}$ times the photon noise, i.e., intrinsic detector noise at the same level as the photon noise, the two adding-up in quadrature. The mapping speed comparison assumes an illustrative time efficiency from the ground of $25 \%$ (due to maintenance, calibration, and discarding of data taken in bad weather conditions). For instance, BICEP2 at the south pole had a total observing efficiency of about 30\% (BICEP2 Collaboration et al. 2014) in the period extending from February 2010 to November 2012, while that of other ground-based experiments has typically been lower. By comparison, the observing efficiency in space is expected to be close to $100 \%$; the PlanckHFI data loss due to glitches ranges from $6 \%$ to $20 \%$ depending on the detector (Planck Collaboration et al. 2014b). Note, however, that such a good observing efficiency requires a continuous sub-kelvin cooler.

Figure 11 shows that, within the atmospheric windows, a single space-borne detector can reach a sensitivity equivalent to 100-200 ground-based detectors (depending on frequency, and ignoring fluctuations of atmospheric emission, ground pickup and other systematics, which further degrade the performance of ground-based observations relatively to space). Outside of the atmospheric windows, i.e., close to the main $\mathrm{O}_{2}$ and $\mathrm{H}_{2} \mathrm{O}$ lines, and above $300 \mathrm{GHz}$, ground-based observations are extremely challenging, and a space mission seems the only option to observe large patches of sky with good sensitivity and with an angular resolution of a few arcminutes or better (i.e., without resorting to massively multi-moded observations).

The sensitivity of space-borne detectors weakly depends on the temperature of the payload. For instance, at $\nu=150 \mathrm{GHz}$, the respective noise levels of a detector in the conditions described above are 49,43 , and $40 \mu \mathrm{K}_{\mathrm{CMB}} \cdot \sqrt{\mathrm{s}}$, respectively. This estimate is slightly better than (but generally in good agreement with) the observed sensitivity of Planck $143 \mathrm{GHz}$ polarisation-sensitive detectors, which range from 50 to $53 \mu \mathrm{K}_{\mathrm{CMB}} \cdot \sqrt{\mathrm{s}}$ (except for one, which is at the level of $59 \mu \mathrm{K}_{\mathrm{CMB}} \cdot \sqrt{\mathrm{s}}$ (Planck HFI Core Team et al. 2011)).

\section{A.2 Required observing time and focal-plane area}

We now assume, for the sake of discussion, that we want to map CMB polarisation at the level of $\sigma=5 \mu \mathrm{K}$.arcmin over the entire sky (i.e., a sky fraction $f_{\text {sky }}=1$ ). Figure 12 gives the number of detector-years of observation required to reach that sensitivity in polarisation, as a function of frequency. The required order of magnitude is 500-1000 detector-years for a space mission, and about $10^{5}$ detector-years on the ground. This number scales proportionally to $1 / \sigma^{2}$ and $f_{\text {sky }}$, so results can straightforwardly be scaled to any required noise level and any observed sky fraction.

Figure 12 also gives an estimate of the focal-plane area $A$ required to reach this full-sky sensitivity after 4 years of observation. For a single-mode pixel observing at wavelength $\lambda$, the focal-plane area required is roughly proportional to $\lambda^{2}$, i.e., $A=\kappa^{2} \lambda^{2}$ where $\kappa^{2}$ is a filling factor that depends on focal-plane technology and on the $F$-number of the telescope. Assuming a filling factor of $9(\kappa=3)$ a focal-plane dimension of order $10 \mathrm{~cm}$ is required in the 

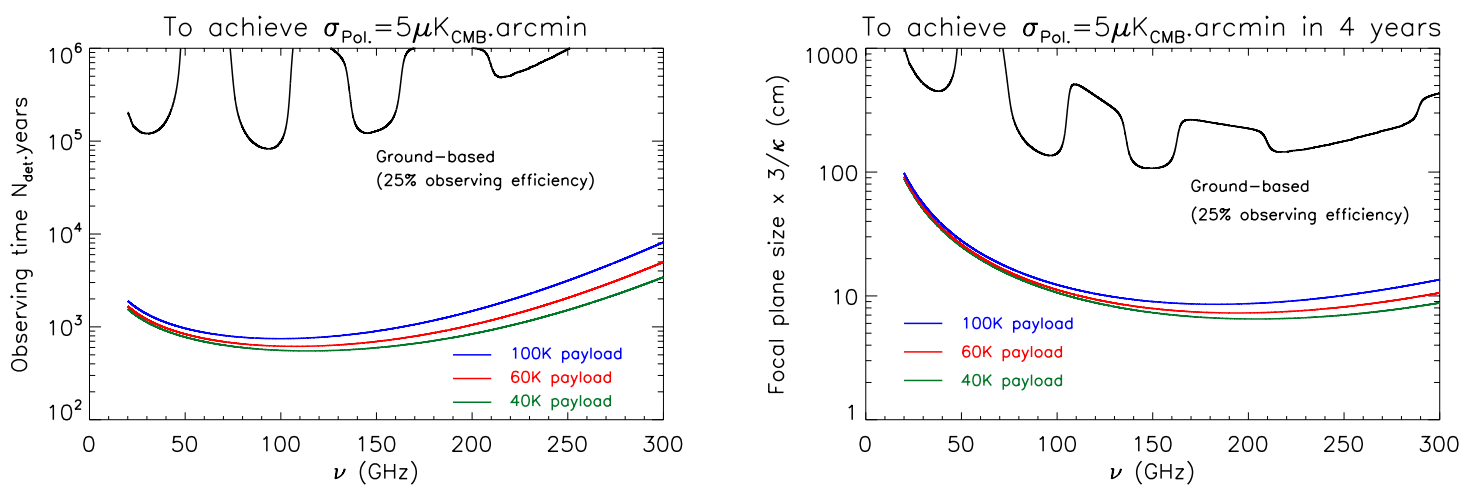

Figure 12. Left: Observing time required to reach a CMB polarisation sensitivity of $5 \mu \mathrm{K}$.arcmin (full sky equivalent) for a ground-based experiment and for a space mission for different assumed payload temperatures. For an observing time of 4 years, about 200-300 detectors in the 100-150 GHz frequency range are required from space, while on the ground about 2 orders of magnitude more detectors are needed. Right: Typical focal-plane size required to reach the same sensitivity, assuming here that $9 \lambda^{2}$ of focal-plane area is required per detector.

focal plane of a space mission, while a focal plane of dimension around $1 \mathrm{~m}$ is required on the ground to reach about the same performance. Multi-frequency, dual-polarisation detectors can help reduce the size of the focal plane (e.g., by a factor $\sqrt{6}$ for dual-polarisation, trichroic detectors), as long as their optical efficiency is not worse than that of single frequency, single polarisation detectors.

From space, the most efficient frequency range (in terms of focal-plane area required to reach a given CMB sensitivity) is between 170 and $200 \mathrm{GHz}$, depending somewhat on the payload temperature (see right panel of figure 12). From the ground, the best observing frequency (from a sensitivity per focal-plane area point of view) is $150 \mathrm{GHz}$.

\section{A.3 Atmospheric emission fluctuations}

In addition to emitting photons that contribute to the total photon noise of the observations, atmospheric emission varies in time and over the sky. These variations are mostly due to inhomogeneities of the precipitable water vapour (clouds) and of the temperature. For an atmosphere at around $300 \mathrm{~K}$ with $2 \%$ emissivity, the total emission is of order $6 \mathrm{~K}_{\mathrm{RJ}}$. Temperature inhomogeneities of $0.1 \%$, for instance, generate fluctuations of the signal of the order of $6 \mathrm{mK}_{\mathrm{RJ}}$. Such fluctuations on the timescale of $1 \mathrm{~s}$ are more than an order of magnitude larger than the $150-\mathrm{GHz}$ detector white noise in typical observing conditions from the Atacama plateau, shown in the bottom left panel of figure 11, and hence are the dominant source of low-frequency noise in the data timestreams. In addition, since such inhomogeneities are correlated over patches of several degrees, the large-scale noise they generate is correlated between the detectors in the focal plane of a single instrument, and does not average down as $\sqrt{N_{\text {det }}}$. However, atmospheric emission is only very weakly polarised. A polarisation modulator, such as a continuously rotating half-wave plate (HWP), typically reduces these fluctuations of atmospheric emission by a factor of around $10^{3}$, so they impact mostly the measurement of temperature anisotropies, and much less polarisation. However, because $B$ modes are more than 3 orders of magnitude below temperature anisotropies, the atmospheric noise cannot be ignored for CMB polarisation measurements on large scales. 
From the ground, observations at frequencies up to $300 \mathrm{GHz}$ are theoretically possible from sites with very low precipitable water vapour and stable observing conditions, such as Antarctica or the Atacama plateau. However, even from such excellent observing sites, broad-band observations at $\nu>300 \mathrm{GHz}$ are very challenging in practice, by reason of these fluctuations of atmospheric emission. Since stratospheric balloons, which can avoid the noise excess due to the atmosphere, are limited to short observing times (e.g., from a few days typically to a few weeks for ultra-long duration ballooning), a space mission is the only viable option to achieve low-noise, few arcminute-angular resolution, large sky area observations at sub-millimetre wavelengths.

\section{B Scan strategy optimisation}

\section{B.1 Main requirements and design drivers}

We assume a scanning strategy such as that described in section 5.3, in which the satellite is spun around its main symmetry axis with a period $T_{\text {spin. }}$. The spin axis precesses around the anti-solar direction with a period $T_{\text {prec }}$. The precession angle is $\alpha$, while the line of sight (LOS) is offset from the principal axis of symmetry by an angle $\beta$. Hence, while the satellite scans the sky, the centre of the field of view (FOV) scans a near-circle of angular radius $\beta$. In this appendix, we discuss the optimisation of $\alpha, \beta, T_{\text {prec }}$, and $T_{\text {spin }}$.

Two general regimes of scanning can be considered. In the first one, where the precession angle $\alpha$ is larger than the spin angle $\beta$, the trajectory of the FOV covers, over the time of a precession period, an annulus for which small circles intersect at large crossing angles. In addition, annuli observed at times separated by about $\beta$ (in degrees) days generate largeangle trajectory crossings for most of the observed pixels. For such a scanning strategy, single detectors observe each pixel at very different angles that are almost evenly spread in $[0,2 \pi]$. Hence, it should be possible to make single-detector maps with good properties of the final covariance of the reconstructed $I, Q, U$ maps: near-nominal noise for both polarisation Stokes parameters; and low cross-correlation between the errors in the three maps. In the second regime, where $\alpha<\beta$, the trajectory of the FOV over one precession period also covers a ring on the sky, but the trajectories cross at smaller angles. Large-angle crossings occur only for data sets obtained at very different times in the mission. It may be necessary to combine several detectors to make polarisation maps, which requires mitigating in the data-analysis step both beam asymmetries and possible bandpass mismatches, i.e., the different response of different detectors to the various astrophysical components present in the sky. The scan geometries for the two scanning regimes are sketched in figure 13.

For the Cosmic Origins Explorer version proposed in answer to the ESA M4 call $(C O r E+)$, the proposed scanning strategy used $\alpha=\beta=45^{\circ}$. In practice, it would be desirable to slightly increase these values (either $\alpha$, or $\beta$, or both), so that all the detectors in the focal plane observe the complete sky. For the CORE version proposed in answer to the M5 call, we set as a baseline $\alpha=30^{\circ}$ and $\beta=65^{\circ}$, so that $\alpha+\beta=95^{\circ}$, which ensures that all the detectors within a focal plane of angular radius of less than $5^{\circ}$ do cover the full sky. We now discuss the rationale for deciding between these options, and how they impact the global design of the payload and the mission.

\section{B.2 Practical constraints}

Thermal stability: The temperature of the payload depends on the incidence angle of solar radiation on the CORE spacecraft. To ensure the thermal stability of the payload, 

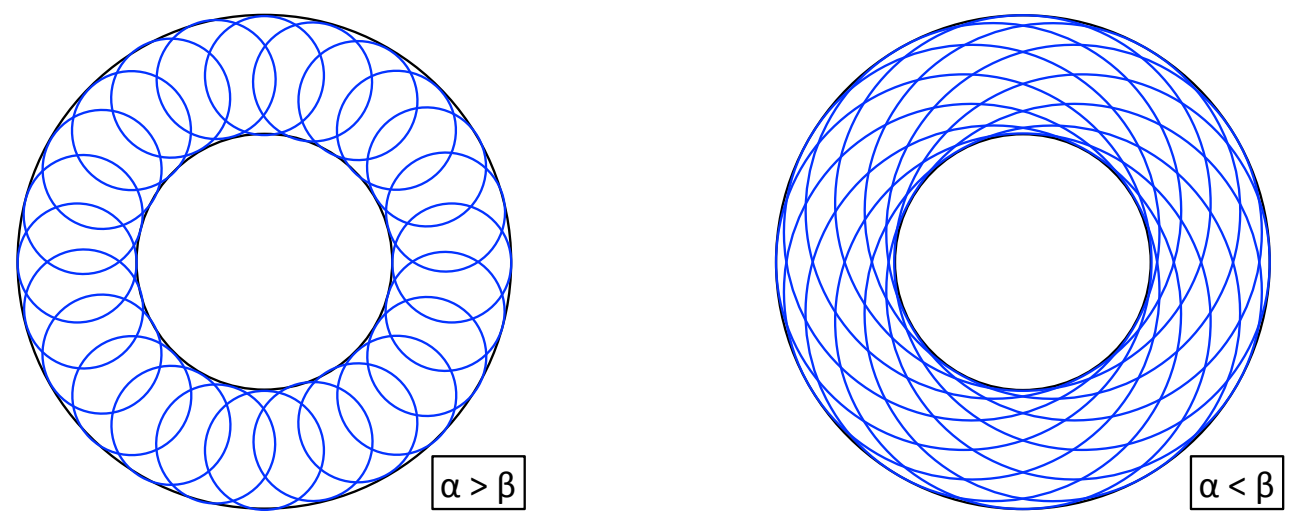

Figure 13. For one complete precession, the FOV observes a ring of width $|\alpha+\beta|-|\alpha-\beta|$. Left: Case where $\alpha>\beta$. Small rings cross at large angles, for a good coverage of angles over timescales of order $\beta$ days, and good distribution of scanning directions for most pixels on the sky over the course of the mission. Right: Case where $\alpha<\beta$. Large rings cross at small angles, but connect very distant pixels on short periods.

the precession axis will be maintained anti-solar (and the payload symmetrical), so that the integrated solar illumination on the solar panels at the bottom of the service module and on the outer "V-groove" screen remains constant.

Sun, Earth and Moon screening: The Sun should never shine on the inner V-groove screens nor on the payload itself. This requirement constrains the precession angle $\alpha$ to be lower than a limiting value $\alpha_{\max }$ set by the geometry of the shields. Similarly the Earth and Moon should never shine directly on the detector array.

Making full-sky maps: The requirement that a given detector $d$ be able to scan the full sky imposes, for an anti-solar precession axis,

$$
\alpha+\beta_{d} \geq 90^{\circ}
$$

where $\beta_{d}=\beta+\Delta \beta_{d}$ is the offset angle of detector $d$ with respect to the spin axis. The angle $\Delta \beta_{d}$ is set by the location of detector $d$ in the focal-plane array and by the optical setup. To make full-sky maps with all detectors, we need

$$
\alpha+\beta \geq 90^{\circ}+\theta_{\mathrm{FP}},
$$

where $\theta_{\mathrm{FP}}$ is the angular radius of the imprint of the focal plane on the sky. Full sky coverage for each individual detector is preferable. For CORE, the field of view is $\lesssim 5^{\circ}$ in radius, so $\alpha+\beta=95^{\circ}$ is appropriate. For much higher values of $\alpha+\beta$, the Sun, Earth, and Moon would come closer to the line of sight, which should be avoided.

Data transfer: Downloading the data to Earth requires pointing a steerable antenna towards the Earth, and hence to cancel the spin and precession of the spacecraft. Either a phased array such as that of Gaia (Allica et al. 2010), or a mechanical pointing system are options. Possible Earth aspect angle $\theta_{\text {Earth }}$ and scanning speed depends on the specifications 
of the antenna (maximum boresight angle and tracking speed). The maximum Earth aspect angle during a precession is:

$$
\theta_{\text {Earth }}=\alpha+\arctan \left(\frac{r_{\text {orbit }}^{\max }}{1.5 \times 10^{6} \mathrm{~km}}\right)
$$

where $r_{\text {orbit }}^{\max }$ is the major axis of the CORE orbit around L2. For example, if we assume $\theta_{\mathrm{Earth}}^{\max }=60^{\circ}$ and $\alpha=30^{\circ}$, the maximum orbital radius is about $860,000 \mathrm{~km}$, compatible with a large Lissajous orbit (see Table 3). For $\alpha=45^{\circ}$ the maximum orbit radius is $400,000 \mathrm{~km}$ (medium Lissajous), and for $\alpha=50^{\circ}$ we get a maximum orbit radius of $260,000 \mathrm{~km}$ (small Lissajous). Injection into a large Lissajous orbit requires lower $\Delta v$ and hence less propellant. This, however, is not a major driver for the mission, since the Ariane 6.2 launcher is designed to carry several tonnes (significantly more than the mass of CORE) to an Earth-escape orbit.

\section{B.3 Sampling}

Co-scan sampling: Denoting as $\theta_{\|}$the sampling angle along the scan (in arcminutes), we have the following equation between the sampling period $T_{\text {sampling }}$ and the spin period $T_{\text {spin }}$ :

$$
\frac{(360 \times 60) \sin \beta}{\theta_{\|}}=\frac{T_{\text {spin }}}{T_{\text {sampling }}} .
$$

For $N_{\mathrm{s}}=4$ samples per beam length along the line of sight, $\beta=65^{\circ}$, and $T_{\text {spin }}=120 \mathrm{~s}$ (CORE proposal baseline), the total number of samples per second for a detector with a $4^{\prime}$ beam is 199, corresponding to a sampling period of about $5 \mathrm{~ms}$.

Cross-scan sampling: For each precession, the trajectory of the spin axis has an angular length of about $(360 \times 60) \sin \alpha$ arcmin. Denoting as $\theta_{\perp}$ the "cross-scan sampling angle," i.e., the maximum distance (in arcminutes) between two consecutive scan paths, as measured along the trajectory of the spin axis, we have

$$
\frac{(360 \times 60) \sin \alpha}{\theta_{\perp}}=\frac{T_{\text {prec }}}{T_{\text {spin }}}
$$

and a cross-scan sampling step of

$$
\left(\frac{\theta_{\perp}}{1^{\prime}}\right)=3.75 \sin \alpha\left(\frac{T_{\text {spin }}}{60 \mathrm{~s}}\right)\left(\frac{96 \mathrm{hr}}{T_{\mathrm{prec}}}\right) .
$$

Ideally, this cross-scan sampling step should also be $\simeq 1 / 4$ of the beam size. For the baseline parameters proposed in response to the M5 call $\left(T_{\text {spin }}=120 \mathrm{~s}, T_{\text {prec }}=4\right.$ day, and $\left.\alpha=30^{\circ}\right)$ we obtain $\theta_{\perp}=3.75^{\prime}$, which is not quite good enough for the highest frequency channels, calling for an increase of the spin rate (1 RPM would be better). We note however that for most of the sky covered by one precession the cross-scan sampling is actually smaller than this maximum value (which is what we obtain for pixels on the trajectory of the spin axis, along the circle of radius $\alpha=30^{\circ}$ centred on the precession axis).

Sky area covered during one precession: The sky area covered by one detector during one precession period is the area of the sphere located between colatitudes $|\alpha-\beta|$ and $(\alpha+\beta)$. The sky fraction $f_{\text {sky }}$ is

$$
f_{\text {sky }}=\frac{1}{2}(\cos |\alpha-\beta|-\cos |\alpha+\beta|) \text {. }
$$

For $\alpha=30^{\circ}$ and $\beta=65^{\circ}$ (proposed baseline), $45 \%$ of the sky is covered for each precession period. 
Data samples per precession: For each precession period, the number of data points for a single detector is

$$
N_{\text {pts } / \text { prec. }} \simeq 4.67 \times 10^{8} \sin \alpha \sin \beta\left(\frac{1^{\prime}}{\theta_{\|}}\right)\left(\frac{1^{\prime}}{\theta_{\perp}}\right) .
$$

The average density of data points per square arcminute for one single precession period is

$$
N_{\mathrm{pts} / \mathrm{arcmin}^{2}} \simeq 6.28\left(\frac{\sin \alpha \sin \beta}{\cos |\alpha-\beta|-\cos |\alpha+\beta|}\right)\left(\frac{1^{\prime}}{\theta_{\|}}\right)\left(\frac{1^{\prime}}{\theta_{\perp}}\right) .
$$
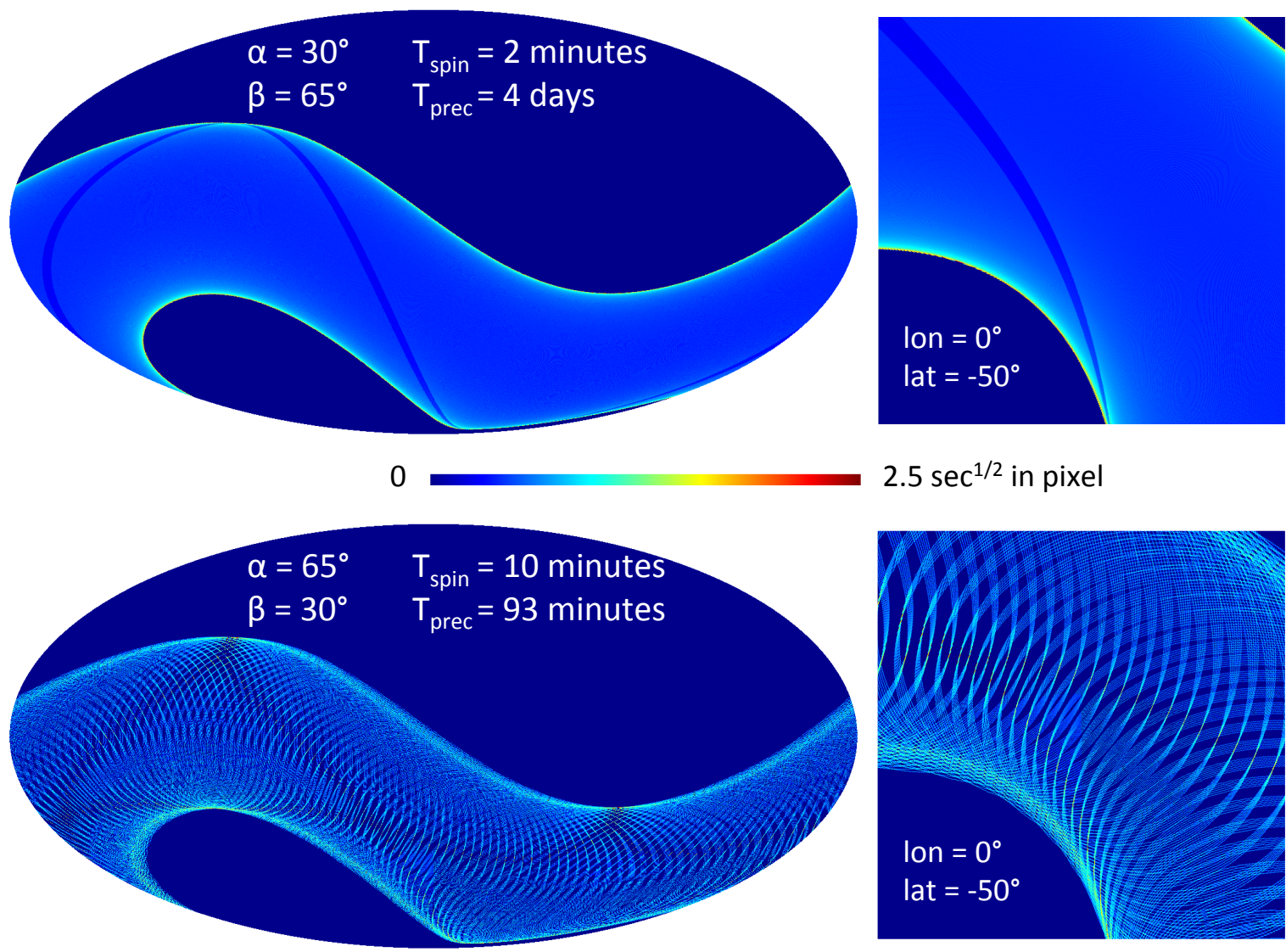

$2.5 \sec ^{1 / 2}$ in pixel

0

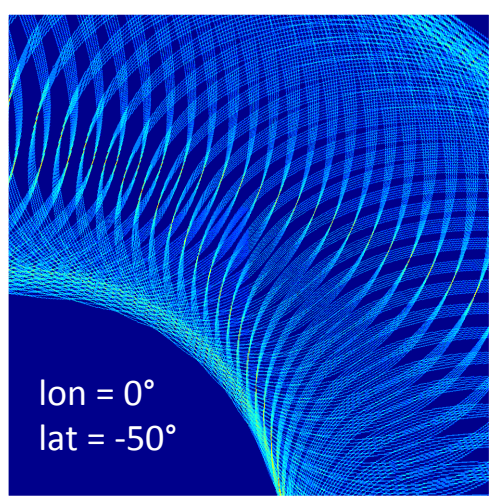

$2.5 \sec ^{1 / 2}$ in pixel

Figure 14. Sky coverage (in units of square root of time in HEALPix map pixels for $N_{\text {side }}=512$ ) for a single detector after 4 days of continuous observation for two choices of parameters for the scanning strategy. Top: Case A, CORE baseline parameters, with precession angle $\alpha=30^{\circ}$, scanning angle $\beta=65^{\circ}$, spin period 2 minutes, and precession period 4 days. Bottom: Case B, LiteBIRD-like scan strategy, with precession angle $\alpha=65^{\circ}$, scanning angle $\beta=30^{\circ}$, spin period 10 minutes, and precession period 93 minutes. Square maps on the right show a detail in the gnomonic projection centred at Galactic coordinates $\left(0^{\circ},-50^{\circ}\right)$. 


\section{B.4 Optimisation}

The optimisation of the scanning strategy must be a compromise between conflicting requirements. We consider as a starting point two concrete examples. The first case that is considered is the CORE baseline (Case A: $\alpha=30^{\circ}, \beta=65^{\circ}, T_{\text {spin }}=120 \mathrm{~s}, T_{\text {prec }}=4$ days), and corresponds to scanning according to the pattern in the right panel of figure 13, while the second case (Case B: $\alpha=65^{\circ}, \beta=30^{\circ}, T_{\text {spin }}=600 \mathrm{~s}, T_{\text {prec }}=93$ minutes) is representative of an option that is considered for LiteBIRD, and corresponds to scanning according to the left panel of figure 13. As discussed in Ref. (Wallis et al. 2017), scan strategy B is nearly ideal in terms of distribution of scan angles on the sky for single-detector map making.

According to eq. (B.7), and as illustrated in figure 13, both sets of angles allow the mission to probe the same sky area over one precession period. The sky-coverage as a function of time, however, also depends on the choice that is made for $T_{\text {prec }} / T_{\text {spin }}$. In the present example, scan strategy A fulfills the requirement to obtain a cross-scan sampling angle small enough compared to the pixel size, while scan strategy B does not.

Figure 14 shows the sky coverage for one single detector after 4 days of observation for both scan strategies, in units of $\sqrt{\mathrm{s}}$ per pixel in a HEALPix $N_{\text {side }}=512$ map in Galactic coordinates. In scan strategy B, large gaps are left between the scans; although they gradually fill up as precessions are accumulated, the distribution of observing time is very inhomogeneous, as clearly seen in the bottom panel of figure 14 .

To match the CORE cross-scan sampling of $3.75^{\prime}$ when $\alpha=65^{\circ}$ and $\beta=30^{\circ}$, we need $T_{\text {prec }} / T_{\text {spin }} \simeq 5220$, which for $T_{\text {spin }}=10$ minutes results in $T_{\text {prec }}=36$ days. If we require a precession period of 4 days, the spin period must be $\simeq 1.1$ minutes instead of 10 .

In principle, it is also possible to adjust the ratio of $T_{\text {prec }} / T_{\text {spin }}$ so that the gaps between the scans fill up optimally as precessions are being accumulated. Still, the total number of spin periods needed to fill in the wide ring is no less than 36 days if $T_{\text {spin }}=10$ minutes. This is not optimal for cross-comparison of maps obtained at different times during the course of the mission. In addition, in 36 days the precession axis moves by about $35.5^{\circ}$ to follow the yearly motion of the Sun, leaving gaps in the sky coverage. To recover proper cross-scan sampling, $T_{\text {spin }}$ must be reduced. We note however that the cross-scan sampling requirement is less stringent for LiteBIRD than for CORE because of its coarser angular resolution.

Optimally, for CORE, we may wish to retain the angles used in scan strategy B, but modify $T_{\text {spin }}$ and $T_{\text {prec }}$. However, as discussed in section $6.7, \alpha=65^{\circ}$ requires additional solar panels and re-defining of the payload $\mathrm{V}$-grooves, and $T_{\text {spin }}=1.1 \mathrm{~min}$ is more demanding on the attitude control system. The parameters chosen for CORE are the result of a compromise between these different constraints.

\section{References}

K. N. Abazajian, P. Adshead, Z. Ahmed, S. W. Allen, D. Alonso, K. S. Arnold, C. Baccigalupi, J. G. Bartlett, N. Battaglia, B. A. Benson, C. A. Bischoff, J. Borrill, V. Buza, E. Calabrese, R. Caldwell, J. E. Carlstrom, C. L. Chang, T. M. Crawford, F.-Y. Cyr-Racine, F. De Bernardis, T. de Haan, S. di Serego Alighieri, J. Dunkley, C. Dvorkin, J. Errard, G. Fabbian, S. Feeney, S. Ferraro, J. P. Filippini, R. Flauger, G. M. Fuller, V. Gluscevic, D. Green, D. Grin, E. Grohs, J. W. Henning, J. C. Hill, R. Hlozek, G. Holder, W. Holzapfel, W. Hu, K. M. Huffenberger, R. Keskitalo, L. Knox, A. Kosowsky, J. Kovac, E. D. Kovetz, C.-L. Kuo, A. Kusaka, M. Le Jeune, A. T. Lee, M. Lilley, M. Loverde, M. S. Madhavacheril, A. Mantz, D. J. E. Marsh, J. McMahon, P. D. Meerburg, J. Meyers, A. D. Miller, J. B. Munoz, H. N. Nguyen, M. D. Niemack, M. Peloso, J. Peloton, L. Pogosian, C. Pryke, M. Raveri, C. L. Reichardt, G. Rocha, A. Rotti, E. Schaan, 
M. M. Schmittfull, D. Scott, N. Sehgal, S. Shandera, B. D. Sherwin, T. L. Smith, L. Sorbo, G. D. Starkman, K. T. Story, A. van Engelen, J. D. Vieira, S. Watson, N. Whitehorn, and W. L. Kimmy Wu. CMB-S4 Science Book, First Edition. ArXiv e-prints, October 2016.

G. E. Addison, Y. Huang, D. J. Watts, C. L. Bennett, M. Halpern, G. Hinshaw, and J. L. Weiland. Quantifying Discordance in the 2015 Planck CMB Spectrum. ApJ, 818:132, February 2016. doi: 10.3847/0004-637X/818/2/132.

J. C. Allica, E. Alonso, M. Amado, A. Bazn, F. Casares, E. de Witte, Q. Garca, T. McConnell, A. Montesano, J. Santilario, and J. L. Serrano. Architecture of gaia satellite phased array antenna. In Proceedings of the Fourth European Conference on Antennas and Propagation, pages 1-4, April 2010.

P. André, C. Baccigalupi, A. Banday, D. Barbosa, B. Barreiro, J. Bartlett, N. Bartolo, E. Battistelli, R. Battye, G. Bendo, A. Benoît, J.-P. Bernard, M. Bersanelli, M. Béthermin, P. Bielewicz, A. Bonaldi, F. Bouchet, F. Boulanger, J. Brand, M. Bucher, C. Burigana, Z.-Y. Cai, P. Camus, F. Casas, V. Casasola, G. Castex, A. Challinor, J. Chluba, G. Chon, S. Colafrancesco, B. Comis, F. Cuttaia, G. D'Alessandro, A. Da Silva, R. Davis, M. de Avillez, P. de Bernardis, M. de Petris, A. de Rosa, G. de Zotti, J. Delabrouille, F.-X. Désert, C. Dickinson, J. M. Diego, J. Dunkley, T. Enßlin, J. Errard, E. Falgarone, P. Ferreira, K. Ferrière, F. Finelli, A. Fletcher, P. Fosalba, G. Fuller, S. Galli, K. Ganga, J. García-Bellido, A. Ghribi, M. Giard, Y. Giraud-Héraud, J. Gonzalez-Nuevo, K. Grainge, A. Gruppuso, A. Hall, J.-C. Hamilton, M. Haverkorn, C. Hernandez-Monteagudo, D. Herranz, M. Jackson, A. Jaffe, R. Khatri, M. Kunz, L. Lamagna, M. Lattanzi, P. Leahy, J. Lesgourgues, M. Liguori, E. Liuzzo, M. Lopez-Caniego, J. Macias-Perez, B. Maffei, D. Maino, A. Mangilli, E. Martinez-Gonzalez, C. J. A. P. Martins, S. Masi, M. Massardi, S. Matarrese, A. Melchiorri, J.-B. Melin, A. Mennella, A. Mignano, M.-A. Miville-Deschênes, A. Monfardini, A. Murphy, P. Naselsky, F. Nati, P. Natoli, M. Negrello, F. Noviello, C. O'Sullivan, F. Paci, L. Pagano, R. Paladino, N. Palanque-Delabrouille, D. Paoletti, H. Peiris, F. Perrotta, F. Piacentini, M. Piat, L. Piccirillo, G. Pisano, G. Polenta, A. Pollo, N. Ponthieu, M. Remazeilles, S. Ricciardi, M. Roman, C. Rosset, J.-A. Rubino-Martin, M. Salatino, A. Schillaci, P. Shellard, J. Silk, A. Starobinsky, R. Stompor, R. Sunyaev, A. Tartari, L. Terenzi, L. Toffolatti, M. Tomasi, N. Trappe, M. Tristram, T. Trombetti, M. Tucci, R. Van de Weijgaert, B. Van Tent, L. Verde, P. Vielva, B. Wandelt, R. Watson, and S. Withington. PRISM (Polarized Radiation Imaging and Spectroscopy Mission): an extended white paper. JCAP, 2:006, February 2014. doi: 10.1088/1475-7516/2014/02/006.

R. Banerji et al. Correction of the effect of bandpass mismatch for future satellite $\mathrm{cmb}$ missions. to be submitted, 2017.

C. L. Bennett, R. S. Hill, G. Hinshaw, D. Larson, K. M. Smith, J. Dunkley, B. Gold, M. Halpern, N. Jarosik, A. Kogut, E. Komatsu, M. Limon, S. S. Meyer, M. R. Nolta, N. Odegard, L. Page, D. N. Spergel, G. S. Tucker, J. L. Weiland, E. Wollack, and E. L. Wright. Seven-year Wilkinson Microwave Anisotropy Probe (WMAP) Observations: Are There Cosmic Microwave Background Anomalies? ApJS, 192:17, February 2011. doi: 10.1088/0067-0049/192/2/17.

BICEP2 Collaboration, P. A. R. Ade, R. W. Aikin, M. Amiri, D. Barkats, S. J. Benton, C. A. Bischoff, J. J. Bock, J. A. Brevik, I. Buder, E. Bullock, G. Davis, P. K. Day, C. D. Dowell, L. Duband, J. P. Filippini, S. Fliescher, S. R. Golwala, M. Halpern, M. Hasselfield, S. R. Hildebrandt, G. C. Hilton, K. D. Irwin, K. S. Karkare, J. P. Kaufman, B. G. Keating, S. A. Kernasovskiy, J. M. Kovac, C. L. Kuo, E. M. Leitch, N. Llombart, M. Lueker, C. B. Netterfield, H. T. Nguyen, R. O'Brient, R. W. Ogburn, IV, A. Orlando, C. Pryke, C. D. Reintsema, S. Richter, R. Schwarz, C. D. Sheehy, Z. K. Staniszewski, K. T. Story, R. V. Sudiwala, G. P. Teply, J. E. Tolan, A. D. Turner, A. G. Vieregg, P. Wilson, C. L. Wong, and K. W. Yoon. BICEP2. II. Experiment and three-year Data Set. ApJ, 792:62, September 2014. doi: 10.1088/0004-637X/792/1/62.

BICEP2 Collaboration, Keck Array Collaboration, P. A. R. Ade, Z. Ahmed, R. W. Aikin, K. D. 
Alexander, D. Barkats, S. J. Benton, C. A. Bischoff, J. J. Bock, R. Bowens-Rubin, J. A. Brevik, I. Buder, E. Bullock, V. Buza, J. Connors, B. P. Crill, L. Duband, C. Dvorkin, J. P. Filippini, S. Fliescher, J. Grayson, M. Halpern, S. Harrison, G. C. Hilton, H. Hui, K. D. Irwin, K. S. Karkare, E. Karpel, J. P. Kaufman, B. G. Keating, S. Kefeli, S. A. Kernasovskiy, J. M. Kovac, C. L. Kuo, E. M. Leitch, M. Lueker, K. G. Megerian, C. B. Netterfield, H. T. Nguyen, R. O'Brient, R. W. Ogburn, A. Orlando, C. Pryke, S. Richter, R. Schwarz, C. D. Sheehy, Z. K. Staniszewski, B. Steinbach, R. V. Sudiwala, G. P. Teply, K. L. Thompson, J. E. Tolan, C. Tucker, A. D. Turner, A. G. Vieregg, A. C. Weber, D. V. Wiebe, J. Willmert, C. L. Wong, W. L. K. Wu, and K. W. Yoon. Improved Constraints on Cosmology and Foregrounds from BICEP2 and Keck Array Cosmic Microwave Background Data with Inclusion of $95 \mathrm{GHz}$ Band. Physical Review Letters, 116(3):031302, January 2016. doi: 10.1103/PhysRevLett.116.031302.

J. Bock, A. Cooray, S. Hanany, B. Keating, A. Lee, T. Matsumura, M. Milligan, N. Ponthieu, T. Renbarger, and H. Tran. The Experimental Probe of Inflationary Cosmology (EPIC): A Mission Concept Study for NASA's Einstein Inflation Probe. ArXiv e-prints, May 2008.

J. Bock, A. Aljabri, A. Amblard, D. Baumann, M. Betoule, T. Chui, L. Colombo, A. Cooray, D. Crumb, P. Day, C. Dickinson, D. Dowell, M. Dragovan, S. Golwala, K. Gorski, S. Hanany, W. Holmes, K. Irwin, B. Johnson, B. Keating, C.-L. Kuo, A. Lee, A. Lange, C. Lawrence, S. Meyer, N. Miller, H. Nguyen, E. Pierpaoli, N. Ponthieu, J.-L. Puget, J. Raab, P. Richards, C. Satter, M. Seiffert, M. Shimon, H. Tran, B. Williams, and J. Zmuidzinas. Study of the Experimental Probe of Inflationary Cosmology (EPIC)-Intemediate Mission for NASA's Einstein Inflation Probe. ArXiv e-prints, June 2009.

F. R. Bouchet, A. Benoît, P. Camus, F. X. Désert, M. Piat, and N. Ponthieu. Charting the New Frontier of the Cosmic Microwave Background Polarization. In F. Casoli, T. Contini, J. M. Hameury, and L. Pagani, editors, SF2A-2005: Semaine de l'Astrophysique Francaise, page 675, December 2005.

M. B. C. Branco, I. Charles, and J. Butterworth. ATHENA X-IFU detector cooling chain. In Space Telescopes and Instrumentation 2014: Ultraviolet to Gamma Ray, volume 9144 of Proc.SPIE, page $91445 \mathrm{~V}$, July 2014. doi: 10.1117/12.2056427.

C. Burigana, C. S. Carvalho, T. Trombetti, A. Notari, M. Quartin, G. De Gasperis, A. Buzzelli, N. Vittorio, G. De Zotti, P. de Bernardis, J. Chluba, M. Bilicki, L. Danese, J. Delabrouille, L. Toffolatti, A. Lapi, M. Negrello, P. Mazzotta, D. Scott, D. Contreras, A. Achucarro, P. Ade, R. Allison, M. Ashdown, M. Ballardini, A. J. Banday, R. Banerji, J. Bartlett, N. Bartolo, S. Basak, M. Bersanelli, A. Bonaldi, M. Bonato, J. Borrill, F. Bouchet, F. Boulanger, T. Brinckmann, M. Bucher, P. Cabella, Z.-Y. Cai, M. Calvo, G. Castellano, A. Challinor, S. Clesse, I. Colantoni, A. Coppolecchia, M. Crook, G. D’Alessandro, J.-M. Diego, A. Di Marco, E. Di Valentino, J. Errard, S. Feeney, R. Fernandez-Cobos, S. Ferraro, F. Finelli, F. Forastieri, S. Galli, R. Genova-Santos, M. Gerbino, J. Gonzalez-Nuevo, S. Grandis, J. Greenslade, S. Hagstotz, S. Hanany, W. Handley, C. Hernandez-Monteagudo, C. Hervias-Caimapo, M. Hills, E. Hivon, K. Kiiveri, T. Kisner, T. Kitching, M. Kunz, H. Kurki-Suonio, L. Lamagna,

A. Lasenby, M. Lattanzi, J. Lesgourgues, M. Liguori, V. Lindholm, M. Lopez-Caniego, G. Luzzi, B. Maffei, N. Mandolesi, E. Martinez-Gonzalez, C. J. A. P. Martins, S. Masi, D. McCarthy, A. Melchiorri, J.-B. Melin, D. Molinari, A. Monfardini, P. Natoli, A. Paiella, D. Paoletti, G. Patanchon, M. Piat, G. Pisano, L. Polastri, G. Polenta, A. Pollo, V. Poulin, M. Remazeilles, M. Roman, J.-A. Rubino-Martin, L. Salvati, A. Tartari, M. Tomasi, D. Tramonte, N. Trappe, C. Tucker, J. Valiviita, R. Van de Weijgaert, B. van Tent, V. Vennin, P. Vielva, K. Young, M. Zannoni, and for the CORE Collaboration. Exploring cosmic origins with CORE: effects of observer peculiar motion. ArXiv e-prints, April 2017.

J. E. Carlstrom, G. P. Holder, and E. D. Reese. Cosmology with the Sunyaev-Zel'dovich Effect. ARA\&A, 40:643-680, 2002. doi: 10.1146/annurev.astro.40.060401.093803.

A. Challinor et al. Exploring cosmic origins with CORE: Gravitational lensing of the cmb. to be 
submitted, 2017.

I. Charles, C. Daniel, J. André, L. Duband, J.-M. Duval, R. den Hartog, K. Mitsuda, K. Shinozaki, H. van Weers, and N. Y. Yamasaki. Preliminary thermal architecture of the X-IFU instrument dewar. In Space Telescopes and Instrumentation 2016: Ultraviolet to Gamma Ray, volume 9905 of Proc.SPIE, page 99052J, July 2016. doi: 10.1117/12.2232710.

B. Collaudin, D. Montet, Y. Roche, S. Ilsen, C. Schamberg, M. Cesa, K. Goodey, J. Rautakoski, C. Jewell, S. Idler, A. Koppe, N. Sonn, D. Hendry, S. Hamer, O. Bauer, H. Feuchtgruber, E. Sawyer, B. Swinyard, S. Sidher, P. Roelfsema, P. Dieleman, and D. Teyssier. Herschel: Testing of Cryogenics Instruments at Spacecraft Level and Early Flight Results. In J. G. Weisend, editor, American Institute of Physics Conference Series, volume 1218 of American Institute of Physics Conference Series, pages 1510-1519, April 2010. doi: 10.1063/1.3422331.

CORE Collaboration, F. Finelli, M. Bucher, A. Achúcarro, M. Ballardini, N. Bartolo, D. Baumann, S. Clesse, J. Errard, W. Handley, M. Hindmarsh, K. Kiiveri, M. Kunz, A. Lasenby, M. Liguori, D. Paoletti, C. Ringeval, J. Väliviita, B. van Tent, V. Vennin, F. Arroja, M. Ashdown, A. J. Banday, R. Banerji, J. Baselmans, J. G. Bartlett, P. de Bernardis, M. Bersanelli, A. Bonaldi, J. Borril, F. R. Bouchet, F. Boulanger, T. Brinckmann, Z.-Y. Cai, M. Calvo, A. Challinor, J. Chluba, G. D’Amico, J. Delabrouille, J. María Diego, G. De Zotti, V. Desjacques, E. Di Valentino, S. Feeney, J. R. Fergusson, S. Ferraro, F. Forastieri, S. Galli, J. García-Bellido, R. T. Génova-Santos, M. Gerbino, J. González-Nuevo, S. Grandis, J. Greenslade, S. Hagstotz, S. Hanany, D. K. Hazra, C. Hernández-Monteagudo, E. Hivon, B. Hu, E. D. Kovetz, H. Kurki-Suonio, M. Lattanzi, J. Lesgourgues, J. Lizarraga, M. López-Caniego, G. Luzzi, B. Maffei, C. J. A. P. Martins, E. Martínez-González, D. McCarthy, S. Matarrese, A. Melchiorri, J.-B. Melin, A. Monfardini, P. Natoli, M. Negrello, F. Oppizzi, E. Pajer, S. P. Patil, M. Piat, G. Pisano, V. Poulin, A. Ravenni, M. Remazeilles, A. Renzi, D. Roest, L. Salvati, A. Tartari, G. Tasinato, J. Torrado, N. Trappe, M. Tucci, J. Urrestilla, P. Vielva, and R. Van de Weygaert. Exploring Cosmic Origins with CORE: Inflation. ArXiv e-prints, December 2016.

F. Couchot, J. Delabrouille, J. Kaplan, and B. Revenu. Optimised polarimeter configurations for measuring the Stokes parameters of the cosmic microwave background radiation. A\&AS, 135: 579-584, March 1999. doi: 10.1051/aas:1999191.

F. Couchot, S. Henrot-Versillé, O. Perdereau, S. Plaszczynski, B. Rouillé d'Orfeuil, M. Spinelli, and M. Tristram. Relieving tensions related to the lensing of the cosmic microwave background temperature power spectra. A\&A, 597:A126, January 2017. doi: 10.1051/0004-6361/201527740.

P. De Bernardis, M. Bucher, C. Burigana, and L. Piccirillo. B-Pol: detecting primordial gravitational waves generated during inflation. Experimental Astronomy, 23:5-16, March 2009. doi: $10.1007 / \mathrm{s} 10686-008-9120-\mathrm{y}$.

P. de Bernardis, P. A. R. Ade, J. J. A. Baselmans, E. S. Battistelli, A. Benoit, M. Bersanelli, A. Bideaud, M. Calvo, F. J. Casas, G. Castellano, A. Catalano, I. Charles, I. Colantoni, F. Columbro, A. Coppolecchia, M. Crook, G. D’Alessandro, M. De Petris, J. Delabrouille, S. Doyle, C. Franceschet, A. Gomez, J. Goupy, S. Hanany, M. Hills, L. Lamagna,

J. Macias-Perez, B. Maffei, S. Martin, E. Martinez-Gonzalez, S. Masi, D. McCarthy, A. Mennella, A. Monfardini, F. Noviello, A. Paiella, F. Piacentini, M. Piat, G. Pisano, G. Signorelli, C. Y. Tan, A. Tartari, N. Trappe, S. Triqueneaux, C. Tucker, G. Vermeulen, K. Young, M. Zannoni, A. Achúcarro, R. Allison, M. Ashdown, M. Ballardini, A. J. Banday, R. Banerji, J. Bartlett, N. Bartolo, S. Basak, A. Bonaldi, M. Bonato, J. Borrill, F. Bouchet, F. Boulanger,

T. Brinckmann, M. Bucher, C. Burigana, A. Buzzelli, Z. Y. Cai, C. S. Carvalho, A. Challinor, J. Chluba, S. Clesse, G. De Gasperis, G. De Zotti, E. Di Valentino, J. M. Diego, J. Errard, S. Feeney, R. Fernandez-Cobos, F. Finelli, F. Forastieri, S. Galli, R. Génova-Santos, M. Gerbino, J. González-Nuevo, S. Hagstotz, J. Greenslade, W. Handley, C. Hernández-Monteagudo, C. Hervias-Caimapo, E. Hivon, K. Kiiveri, T. Kisner, T. Kitching, M. Kunz, H. Kurki-Suonio, A. Lasenby, M. Lattanzi, J. Lesgourgues, A. Lewis, M. Liguori, V. Lindholm, G. Luzzi, C. J. A. P. 
Martins, A. Melchiorri, J. B. Melin, D. Molinari, P. Natoli, M. Negrello, A. Notari, D. Paoletti, G. Patanchon, L. Polastri, G. Polenta, A. Pollo, V. Poulin, M. Quartin, M. Remazeilles, M. Roman, J. A. Rubiño-Martín, L. Salvati, M. Tomasi, D. Tramonte, T. Trombetti, J. Väliviita, R. Van de Weijgaert, B. van Tent, V. Vennin, P. Vielva, N. Vittorio, and for the CORE collaboration. Exploring Cosmic Origins with CORE: The Instrument. ArXiv e-prints, May 2017.

G. de Gasperis, A. Balbi, P. Cabella, P. Natoli, and N. Vittorio. ROMA: A map-making algorithm for polarised CMB data sets. A\&A, 436:1159-1165, June 2005. doi: 10.1051/0004-6361:20042512.

G. De Zotti, J. Gonzalez-Nuevo, M. Lopez-Caniego, M. Negrello, J. Greenslade, C. Hernandez-Monteagudo, J. Delabrouille, Z.-Y. Cai, M. Biesiada, M. Bilicki, A. Bonaldi, M. Bonato, C. Burigana, D. L. Clements, S. Colafrancesco, J. M. Diego, A. Le Brun, M. Massardi, J. B. Melin, A. Pollo, B. Roukema, S. Serjeant, L. Toffolatti, M. Tucci, and the CORE collaboration. Exploring Cosmic Origins with CORE: Extragalactic sources in Cosmic Microwave Background maps. ArXiv e-prints, September 2016.

J. Delabrouille and J.-F. Cardoso. Diffuse Source Separation in CMB Observations. In V. J. Martínez, E. Saar, E. Martínez-González, and M.-J. Pons-Bordería, editors, Data Analysis in Cosmology, volume 665 of Lecture Notes in Physics, Berlin Springer Verlag, pages 159-205, 2009. doi: 10.1007/978-3-540-44767-2_6.

J. Delabrouille, G. Patanchon, and E. Audit. Separation of instrumental and astrophysical foregrounds for mapping cosmic microwave background anisotropies. MNRAS, 330:807-816, March 2002. doi: 10.1046/j.1365-8711.2002.05200.x.

E. Di Valentino, T. Brinckmann, M. Gerbino, V. Poulin, F. R. Bouchet, J. Lesgourgues, A. Melchiorri, J. Chluba, S. Clesse, J. Delabrouille, C. Dvorkin, F. Forastieri, S. Galli, D. C. Hooper, M. Lattanzi, C. J. A. P. Martins, L. Salvati, G. Cabass, A. Caputo, E. Giusarma, E. Hivon, P. Natoli, L. Pagano, S. Paradiso, J. A. Rubino-Martin, A. Achucarro, M. Ballardini, N. Bartolo, D. Baumann, J. G. Bartlett, P. de Bernardis, A. Bonaldi, M. Bucher, Z.-Y. Cai, G. De Zotti, J. M. Diego, J. Errard, S. Ferraro, F. Finelli, R. T. Genova-Santos, J. Gonzalez-Nuevo, S. Grandis, J. Greenslade, S. Hagstotz, W. Handley, M. Hindmarsh, C. Hernandez-Monteagudo, K. Kiiveri, M. Kunz, A. Lasenby, M. Liguori, M. Lopez-Caniego, G. Luzzi, J.-B. Melin, J. J. Mohr, M. Negrello, D. Paoletti, M. Remazeilles, C. Ringeval, J. Valiviita, B. Van Tent, V. Vennin, N. Vittorio, and the CORE collaboration. Exploring Cosmic Origins with CORE: Cosmological Parameters. ArXiv e-prints, November 2016.

H. K. Eriksen, F. K. Hansen, A. J. Banday, K. M. Górski, and P. B. Lilje. Asymmetries in the Cosmic Microwave Background Anisotropy Field. ApJ, 605:14-20, April 2004. doi: $10.1086 / 382267$.

J. Errard, P. A. R. Ade, Y. Akiba, K. Arnold, M. Atlas, C. Baccigalupi, D. Barron, D. Boettger, J. Borrill, S. Chapman, Y. Chinone, A. Cukierman, J. Delabrouille, M. Dobbs, A. Ducout, T. Elleflot, G. Fabbian, C. Feng, S. Feeney, A. Gilbert, N. Goeckner-Wald, N. W. Halverson, M. Hasegawa, K. Hattori, M. Hazumi, C. Hill, W. L. Holzapfel, Y. Hori, Y. Inoue, G. C. Jaehnig, A. H. Jaffe, O. Jeong, N. Katayama, J. Kaufman, B. Keating, Z. Kermish, R. Keskitalo, T. Kisner, M. Le Jeune, A. T. Lee, E. M. Leitch, D. Leon, E. Linder, F. Matsuda, T. Matsumura, N. J. Miller, M. J. Myers, M. Navaroli, H. Nishino, T. Okamura, H. Paar, J. Peloton, D. Poletti, G. Puglisi, G. Rebeiz, C. L. Reichardt, P. L. Richards, C. Ross, K. M. Rotermund, D. E. Schenck, B. D. Sherwin, P. Siritanasak, G. Smecher, N. Stebor, B. Steinbach, R. Stompor, A. Suzuki, O. Tajima, S. Takakura, A. Tikhomirov, T. Tomaru, N. Whitehorn, B. Wilson, A. Yadav, and O. Zahn. Modeling Atmospheric Emission for CMB Ground-based Observations. ApJ, 809:63, August 2015. doi: 10.1088/0004-637X/809/1/63.

T. Essinger-Hileman, A. Kusaka, J. W. Appel, S. K. Choi, K. Crowley, S. P. Ho, N. Jarosik, L. A. Page, L. P. Parker, S. Raghunathan, S. M. Simon, S. T. Staggs, and K. Visnjic. Systematic effects from an ambient-temperature, continuously rotating half-wave plate. Review of Scientific Instruments, 87(9):094503, September 2016. doi: 10.1063/1.4962023. 
S. Galli, K. Benabed, F. Bouchet, J.-F. Cardoso, F. Elsner, E. Hivon, A. Mangilli, S. Prunet, and B. Wandelt. CMB polarization can constrain cosmology better than CMB temperature. Phys.Rev.D, 90(6):063504, September 2014. doi: 10.1103/PhysRevD.90.063504.

K. M. Górski, E. Hivon, A. J. Banday, B. D. Wandelt, F. K. Hansen, M. Reinecke, and M. Bartelmann. HEALPix: A Framework for High-Resolution Discretization and Fast Analysis of Data Distributed on the Sphere. ApJ, 622:759-771, April 2005. doi: 10.1086/427976.

Q. Guo, S. White, C. Li, and M. Boylan-Kolchin. How do galaxies populate dark matter haloes? MNRAS, 404:1111-1120, May 2010. doi: 10.1111/j.1365-2966.2010.16341.x.

C. A. Hill, S. Beckman, Y. Chinone, N. Goeckner-Wald, M. Hazumi, B. Keating, A. Kusaka, A. T. Lee, F. Matsuda, R. Plambeck, A. Suzuki, and S. Takakura. Design and development of an ambient-temperature continuously-rotating achromatic half-wave plate for CMB polarization modulation on the POLARBEAR-2 experiment. In Millimeter, Submillimeter, and Far-Infrared Detectors and Instrumentation for Astronomy VIII, volume 9914 of Proc.SPIE, page 99142U, July 2016. doi: $10.1117 / 12.2232280$.

W. Hu and T. Okamoto. Mass Reconstruction with Cosmic Microwave Background Polarization. ApJ, 574:566-574, August 2002. doi: 10.1086/341110.

H. Ishino, Y. Akiba, K. Arnold, D. Barron, J. Borrill, R. Chendra, Y. Chinone, S. Cho,

A. Cukierman, T. de Haan, M. Dobbs, A. Dominjon, T. Dotani, T. Elleflot, J. Errard, T. Fujino, H. Fuke, T. Funaki, N. Goeckner-Wald, N. Halverson, P. Harvey, T. Hasebe, M. Hasegawa, K. Hattori, M. Hattori, M. Hazumi, N. Hidehira, C. Hill, G. Hilton, W. Holzapfel, Y. Hori, J. Hubmayr, K. Ichiki, H. Imada, J. Inatani, M. Inoue, Y. Inoue, F. Irie, K. Irwin, H. Ishitsuka, O. Jeong, H. Kanai, K. Karatsu, S. Kashima, N. Katayama, I. Kawano, T. Kawasaki, B. Keating, S. Kernasovskiy, R. Keskitalo, A. Kibayashi, Y. Kida, N. Kimura, K. Kimura, T. Kisner, K. Kohri, E. Komatsu, K. Komatsu, C.-L. Kuo, S. Kuromiya, A. Kusaka, A. Lee, D. Li, E. Linder, M. Maki, H. Matsuhara, T. Matsumura, S. Matsuoka, S. Matsuura, S. Mima, Y. Minami, K. Mitsuda, M. Nagai, T. Nagasaki, R. Nagata, M. Nakajima, S. Nakamura, T. Namikawa, M. Naruse, T. Nishibori, K. Nishijo, H. Nishino, A. Noda, T. Noguchi, H. Ogawa, W. Ogburn, S. Oguri, I. Ohta, N. Okada, A. Okamoto, T. Okamura, C. Otani, G. Pisano, G. Rebeiz, P. Richards, S. Sakai, Y. Sakurai, Y. Sato, N. Sato, Y. Segawa, S. Sekiguchi, Y. Sekimoto, M. Sekine, U. Seljak, B. Sherwin, T. Shimizu, K. Shinozaki, S. Shu, R. Stompor, H. Sugai, H. Sugita, J. Suzuki, T. Suzuki, A. Suzuki, O. Tajima, S. Takada, S. Takakura, K. Takano, S. Takatori, Y. Takei, D. Tanabe, T. Tomaru, N. Tomita, P. Turin, S. Uozumi, S. Utsunomiya, Y. Uzawa, T. Wada, H. Watanabe, B. Westbrook, N. Whitehorn, Y. Yamada, R. Yamamoto, N. Yamasaki, T. Yamashita, T. Yoshida, M. Yoshida, and K. Yotsumoto. LiteBIRD: lite satellite for the study of B-mode polarization and inflation from cosmic microwave background radiation detection. In Society of Photo-Optical Instrumentation Engineers (SPIE) Conference Series, volume 9904 of Proc.SPIE, page 99040X, July 2016. doi: 10.1117/12.2231995.

M. Kamionkowski and E. D. Kovetz. The Quest for B Modes from Inflationary Gravitational Waves. ARA\&A, 54:227-269, September 2016. doi: 10.1146/annurev-astro-081915-023433.

M. Kamionkowski, A. Kosowsky, and A. Stebbins. Statistics of cosmic microwave background polarization. Phys.Rev.D, 55:7368-7388, June 1997. doi: 10.1103/PhysRevD.55.7368.

M. Kaplinghat, L. Knox, and Y.-S. Song. Determining Neutrino Mass from the Cosmic Microwave Background Alone. Physical Review Letters, 91(24):241301, December 2003. doi: 10.1103/PhysRevLett.91.241301.

R. Keisler, S. Hoover, N. Harrington, J. W. Henning, P. A. R. Ade, K. A. Aird, J. E. Austermann, J. A. Beall, A. N. Bender, B. A. Benson, L. E. Bleem, J. E. Carlstrom, C. L. Chang, H. C. Chiang, H.-M. Cho, R. Citron, T. M. Crawford, A. T. Crites, T. de Haan, M. A. Dobbs, W. Everett, J. Gallicchio, J. Gao, E. M. George, A. Gilbert, N. W. Halverson, D. Hanson, G. C. Hilton, G. P. Holder, W. L. Holzapfel, Z. Hou, J. D. Hrubes, N. Huang, J. Hubmayr, K. D. Irwin, 
L. Knox, A. T. Lee, E. M. Leitch, D. Li, D. Luong-Van, D. P. Marrone, J. J. McMahon, J. Mehl, S. S. Meyer, L. Mocanu, T. Natoli, J. P. Nibarger, V. Novosad, S. Padin, C. Pryke, C. L. Reichardt, J. E. Ruhl, B. R. Saliwanchik, J. T. Sayre, K. K. Schaffer, E. Shirokoff, G. Smecher, A. A. Stark, K. T. Story, C. Tucker, K. Vanderlinde, J. D. Vieira, G. Wang, N. Whitehorn, V. Yefremenko, and O. Zahn. Measurements of Sub-degree B-mode Polarization in the Cosmic Microwave Background from 100 Square Degrees of SPTpol Data. ApJ, 807:151, July 2015. doi: 10.1088/0004-637X/807/2/151.

A. Kogut, D. J. Fixsen, D. T. Chuss, J. Dotson, E. Dwek, M. Halpern, G. F. Hinshaw, S. M. Meyer, S. H. Moseley, M. D. Seiffert, D. N. Spergel, and E. J. Wollack. The Primordial Inflation Explorer (PIXIE): a nulling polarimeter for cosmic microwave background observations. JCAP, 7:025, July 2011. doi: 10.1088/1475-7516/2011/07/025.

A. Kogut, J. Chluba, D. J. Fixsen, S. Meyer, and D. Spergel. The Primordial Inflation Explorer (PIXIE). In Society of Photo-Optical Instrumentation Engineers (SPIE) Conference Series, volume 9904 of Proc.SPIE, page 99040W, July 2016. doi: 10.1117/12.2231090.

H. Kurki-Suonio, E. Keihänen, R. Keskitalo, T. Poutanen, A.-S. Sirviö, D. Maino, and C. Burigana. Destriping CMB temperature and polarization maps. A\&A, 506:1511-1539, November 2009. doi: 10.1051/0004-6361/200912361.

A. Kusaka, T. Essinger-Hileman, J. W. Appel, P. Gallardo, K. D. Irwin, N. Jarosik, M. R. Nolta, L. A. Page, L. P. Parker, S. Raghunathan, J. L. Sievers, S. M. Simon, S. T. Staggs, and K. Visnjic. Modulation of cosmic microwave background polarization with a warm rapidly rotating half-wave plate on the Atacama B-Mode Search instrument. Review of Scientific Instruments, 85(2):024501, February 2014. doi: 10.1063/1.4862058.

A. Lewis and A. Challinor. Weak gravitational lensing of the CMB. Phys.Rep., 429:1-65, June 2006. doi: 10.1016/j.physrep.2006.03.002.

D. H. Lyth. What Would We Learn by Detecting a Gravitational Wave Signal in the Cosmic Microwave Background Anisotropy? Physical Review Letters, 78:1861-1863, March 1997. doi: 10.1103/PhysRevLett.78.1861.

T. Matsumura, Y. Akiba, J. Borrill, Y. Chinone, M. Dobbs, H. Fuke, A. Ghribi, M. Hasegawa, K. Hattori, M. Hattori, M. Hazumi, W. Holzapfel, Y. Inoue, K. Ishidoshiro, H. Ishino, H. Ishitsuka, K. Karatsu, N. Katayama, I. Kawano, A. Kibayashi, Y. Kibe, K. Kimura, N. Kimura, K. Koga, M. Kozu, E. Komatsu, A. Lee, H. Matsuhara, S. Mima, K. Mitsuda, K. Mizukami, H. Morii, T. Morishima, S. Murayama, M. Nagai, R. Nagata, S. Nakamura, M. Naruse, K. Natsume, T. Nishibori, H. Nishino, A. Noda, T. Noguchi, H. Ogawa, S. Oguri, I. Ohta, C. Otani, P. Richards, S. Sakai, N. Sato, Y. Sato, Y. Sekimoto, A. Shimizu, K. Shinozaki, H. Sugita, T. Suzuki, A. Suzuki, O. Tajima, S. Takada, S. Takakura, Y. Takei, T. Tomaru, Y. Uzawa, T. Wada, H. Watanabe, M. Yoshida, N. Yamasaki, T. Yoshida, and K. Yotsumoto. Mission Design of LiteBIRD. Journal of Low Temperature Physics, 176:733-740, September 2014. doi: 10.1007/s10909-013-0996-1.

J.-B. Melin, A. Bonaldi, M. Remazeilles, S. Hagstotz, J. M. Diego, C. Hernández-Monteagudo, R. T. Génova-Santos, G. Luzzi, C. J. A. P. Martins, S. Grandis, J. J. Mohr, J. G. Bartlett,

J. Delabrouille, S. Ferraro, D. Tramonte, J. A. Rubiño-Martín, J. F. Macias-Pérez, A. Achúcarro, P. Ade, R. Allison, M. Ashdown, M. Ballardini, A. J. Banday, R. Banerji, N. Bartolo, S. Basak, J. Baselmans, K. Basu, R. A. Battye, D. Baumann, M. Bersanelli, M. Bonato, J. Borrill, F. Bouchet, F. Boulanger, T. Brinckmann, M. Bucher, C. Burigana, A. Buzzelli, Z.-Y. Cai, M. Calvo, C. S. Carvalho, M. G. Castellano, A. Challinor, J. Chluba, S. Clesse, S. Colafrancesco, I. Colantoni, A. Coppolecchia, M. Crook, G. D’Alessandro, P. de Bernardis, G. de Gasperis, M. De Petris, G. De Zotti, E. Di Valentino, J. Errard, S. M. Feeney, R. Fernández-Cobos, F. Finelli, F. Forastieri, S. Galli, M. Gerbino, J. González-Nuevo, J. Greenslade, S. Hanany, W. Handley, C. Hervias-Caimapo, M. Hills, E. Hivon, K. Kiiveri, T. Kisner, T. Kitching, M. Kunz, H. Kurki-Suonio, L. Lamagna, A. Lasenby, M. Lattanzi, A. M. C. Le Brun, J. Lesgourgues, 
A. Lewis, M. Liguori, V. Lindholm, M. Lopez-Caniego, B. Maffei, E. Martinez-Gonzalez, S. Masi, D. McCarthy, A. Melchiorri, D. Molinari, A. Monfardini, P. Natoli, M. Negrello, A. Notari, A. Paiella, D. Paoletti, G. Patanchon, M. Piat, G. Pisano, L. Polastri, G. Polenta, A. Pollo, V. Poulin, M. Quartin, M. Roman, L. Salvati, A. Tartari, M. Tomasi, N. Trappe, S. Triqueneaux, T. Trombetti, C. Tucker, J. Väliviita, R. van de Weygaert, B. Van Tent, V. Vennin, P. Vielva, N. Vittorio, J. Weller, K. Young, M. Zannoni, and for the CORE collaboration. Exploring Cosmic Origins with CORE: Cluster Science. ArXiv e-prints, March 2017.

L. Moncelsi, P. A. R. Ade, F. E. Angilè, S. J. Benton, M. J. Devlin, L. M. Fissel, N. N. Gandilo, J. O. Gundersen, T. G. Matthews, C. B. Netterfield, G. Novak, D. Nutter, E. Pascale, F. Poidevin, G. Savini, D. Scott, J. D. Soler, L. D. Spencer, M. D. P. Truch, G. S. Tucker, and J. Zhang. Empirical modelling of the BLASTPol achromatic half-wave plate for precision submillimetre polarimetry. MNRAS, 437:2772-2789, January 2014. doi: 10.1093/mnras/stt2090.

K. Nakamura and S. T. Petcov. Neutrino Mass, Mixing, and Oscillations. In C. Patrignani, editor, Review of Particle Physics, volume 40 of Chin. Phys. C, page 100001, 2016. doi: 10.1088/1674-1137/40/10/090001.

P. Natoli et al. Exploring cosmic origins with CORE: Mitigation of systematic effects. to be submitted, 2017.

M. D. Niemack. Designs for a large-aperture telescope to map the CMB 10 times faster. Appl.Optics, 55:1686, March 2016. doi: 10.1364/AO.55.001686.

E. N. Parker. Cosmical magnetic fields: Their origin and their activity. Clarendon Press, Oxford, 1979.

G. Patanchon, P. A. R. Ade, J. J. Bock, E. L. Chapin, M. J. Devlin, S. Dicker, M. Griffin, J. O. Gundersen, M. Halpern, P. C. Hargrave, D. H. Hughes, J. Klein, G. Marsden, P. G. Martin, P. Mauskopf, C. B. Netterfield, L. Olmi, E. Pascale, M. Rex, D. Scott, C. Semisch, M. D. P. Truch, C. Tucker, G. S. Tucker, M. P. Viero, and D. V. Wiebe. SANEPIC: A Mapmaking Method for Time Stream Data from Large Arrays. ApJ, 681:708-725, July 2008. doi: 10.1086/588543.

Planck Collaboration. Planck intermediate results. XXXIII. Signature of the magnetic field geometry of interstellar filaments in dust polarization maps. A\&A, 586:A136, February 2016a. doi: 10.1051/0004-6361/201425305.

Planck Collaboration. Planck intermediate results. XXXIV. The magnetic field structure in the Rosette Nebula. A\&A, 586:A137, February 2016b. doi: 10.1051/0004-6361/201525616.

Planck Collaboration. Planck intermediate results. XXXV. Probing the role of the magnetic field in the formation of structure in molecular clouds. A\&A, 586:A138, February 2016c. doi: 10.1051/0004-6361/201525896.

Planck Collaboration. Planck intermediate results. XXXVIII. E- and B-modes of dust polarization from the magnetized filamentary structure of the interstellar medium. A\&A, 586:A141, February 2016d. doi: 10.1051/0004-6361/201526506.

Planck Collaboration. Planck intermediate results. XLII. Large-scale Galactic magnetic fields. A\&A, 596:A103, December 2016e. doi: 10.1051/0004-6361/201528033.

Planck Collaboration. Planck intermediate results. XLIV. Structure of the Galactic magnetic field from dust polarization maps of the southern Galactic cap. A\&A, 596:A105, December 2016f. doi: 10.1051/0004-6361/201628636.

Planck Collaboration. Planck 2016 intermediate results. LI. Features in the cosmic microwave background temperature power spectrum and shifts in cosmological parameters. ArXiv e-prints, August 2016g.

Planck Collaboration, P. A. R. Ade, N. Aghanim, M. Arnaud, M. Ashdown, J. Aumont, C. Baccigalupi, M. Baker, A. Balbi, A. J. Banday, and et al. Planck early results. II. The thermal performance of Planck. A\&A, 536:A2, December 2011. doi: 10.1051/0004-6361/201116486. 
Planck Collaboration, P. A. R. Ade, N. Aghanim, M. I. R. Alves, C. Armitage-Caplan, M. Arnaud, M. Ashdown, F. Atrio-Barandela, J. Aumont, C. Baccigalupi, and et al. Planck 2013 results. XIII. Galactic CO emission. A\&A, 571:A13, November 2014a. doi: 10.1051/0004-6361/201321553.

Planck Collaboration, P. A. R. Ade, N. Aghanim, C. Armitage-Caplan, M. Arnaud, M. Ashdown, F. Atrio-Barandela, J. Aumont, C. Baccigalupi, A. J. Banday, and et al. Planck 2013 results. X. HFI energetic particle effects: characterization, removal, and simulation. A\&A, 571:A10, November 2014b. doi: 10.1051/0004-6361/201321577.

Planck Collaboration, P. A. R. Ade, N. Aghanim, C. Armitage-Caplan, M. Arnaud, M. Ashdown, F. Atrio-Barandela, J. Aumont, C. Baccigalupi, A. J. Banday, and et al. Planck 2013 results. XIV. Zodiacal emission. A\&A, 571:A14, November 2014c. doi: 10.1051/0004-6361/201321562.

Planck Collaboration, P. A. R. Ade, N. Aghanim, C. Armitage-Caplan, M. Arnaud, M. Ashdown, F. Atrio-Barandela, J. Aumont, C. Baccigalupi, A. J. Banday, and et al. Planck 2013 results. XVI. Cosmological parameters. A\&A, 571:A16, November 2014d. doi: 10.1051/0004-6361/201321591.

Planck Collaboration, P. A. R. Ade, N. Aghanim, C. Armitage-Caplan, M. Arnaud, M. Ashdown, F. Atrio-Barandela, J. Aumont, C. Baccigalupi, A. J. Banday, and et al. Planck 2013 results. XX. Cosmology from Sunyaev-Zeldovich cluster counts. A\&A, 571:A20, November 2014e. doi: 10.1051/0004-6361/201321521.

Planck Collaboration, P. A. R. Ade, N. Aghanim, C. Armitage-Caplan, M. Arnaud, M. Ashdown, F. Atrio-Barandela, J. Aumont, C. Baccigalupi, A. J. Banday, and et al. Planck 2013 results. XXII. Constraints on inflation. A\&A, 571:A22, November 2014f. doi: 10.1051/0004-6361/201321569.

Planck Collaboration, P. A. R. Ade, N. Aghanim, C. Armitage-Caplan, M. Arnaud, M. Ashdown, F. Atrio-Barandela, J. Aumont, C. Baccigalupi, A. J. Banday, and et al. Planck 2013 results. XXIII. Isotropy and statistics of the CMB. A\&A, 571:A23, November 2014g. doi: 10.1051/0004-6361/201321534.

Planck Collaboration, R. Adam, P. A. R. Ade, N. Aghanim, Y. Akrami, M. I. R. Alves, F. Argüeso, M. Arnaud, F. Arroja, M. Ashdown, and et al. Planck 2015 results. I. Overview of products and scientific results. A\&A, 594:A1, September 2016a. doi: 10.1051/0004-6361/201527101.

Planck Collaboration, R. Adam, P. A. R. Ade, N. Aghanim, M. I. R. Alves, M. Arnaud, D. Arzoumanian, M. Ashdown, J. Aumont, C. Baccigalupi, and et al. Planck intermediate results. XXXII. The relative orientation between the magnetic field and structures traced by interstellar dust. A\&A, 586:A135, February 2016b. doi: 10.1051/0004-6361/201425044.

Planck Collaboration, R. Adam, P. A. R. Ade, N. Aghanim, M. Arnaud, J. Aumont, C. Baccigalupi, A. J. Banday, R. B. Barreiro, J. G. Bartlett, and et al. Planck intermediate results. XXX. The angular power spectrum of polarized dust emission at intermediate and high Galactic latitudes. A\&A, 586:A133, February 2016c. doi: 10.1051/0004-6361/201425034.

Planck Collaboration, P. A. R. Ade, N. Aghanim, M. Arnaud, F. Arroja, M. Ashdown, J. Aumont, C. Baccigalupi, M. Ballardini, A. J. Banday, and et al. Planck 2015 results. XX. Constraints on inflation. A\&A, 594:A20, September 2016d. doi: 10.1051/0004-6361/201525898.

Planck Collaboration, P. A. R. Ade, N. Aghanim, M. Arnaud, M. Ashdown, J. Aumont, C. Baccigalupi, A. J. Banday, R. B. Barreiro, J. G. Bartlett, and et al. Planck 2015 results. XIII. Cosmological parameters. A\&A, 594:A13, September 2016e. doi: 10.1051/0004-6361/201525830.

Planck Collaboration, P. A. R. Ade, N. Aghanim, M. Arnaud, M. Ashdown, J. Aumont, C. Baccigalupi, A. J. Banday, R. B. Barreiro, J. G. Bartlett, and et al. Planck 2015 results. XV. Gravitational lensing. A\&A, 594:A15, September 2016f. doi: 10.1051/0004-6361/201525941.

Planck Collaboration, P. A. R. Ade, N. Aghanim, M. Arnaud, M. Ashdown, J. Aumont, C. Baccigalupi, A. J. Banday, R. B. Barreiro, J. G. Bartlett, and et al. Planck 2015 results. 
XXIV. Cosmology from Sunyaev-Zeldovich cluster counts. A\&A, 594:A24, September 2016g. doi: 10.1051/0004-6361/201525833.

Planck Collaboration, N. Aghanim, M. Ashdown, J. Aumont, C. Baccigalupi, M. Ballardini, A. J. Banday, R. B. Barreiro, N. Bartolo, S. Basak, K. Benabed, J.-P. Bernard, M. Bersanelli, P. Bielewicz, L. Bonavera, J. R. Bond, J. Borrill, F. R. Bouchet, F. Boulanger, C. Burigana, E. Calabrese, J.-F. Cardoso, J. Carron, H. C. Chiang, L. P. L. Colombo, B. Comis, F. Couchot, A. Coulais, B. P. Crill, A. Curto, F. Cuttaia, P. de Bernardis, G. de Zotti, J. Delabrouille, E. Di Valentino, C. Dickinson, J. M. Diego, O. Doré, M. Douspis, A. Ducout, X. Dupac, S. Dusini, F. Elsner, T. A. Enßlin, H. K. Eriksen, E. Falgarone, Y. Fantaye, F. Finelli, F. Forastieri, M. Frailis, A. A. Fraisse, E. Franceschi, A. Frolov, S. Galeotta, S. Galli, K. Ganga, R. T. Génova-Santos, M. Gerbino, T. Ghosh, Y. Giraud-Héraud, J. González-Nuevo, K. M. Górski, A. Gruppuso, J. E. Gudmundsson, F. K. Hansen, G. Helou, S. Henrot-Versillé, D. Herranz, E. Hivon, Z. Huang, A. H. Jaffe, W. C. Jones, E. Keihänen, R. Keskitalo, K. Kiiveri, T. S. Kisner, N. Krachmalnicoff, M. Kunz, H. Kurki-Suonio, J.-M. Lamarre, M. Langer, A. Lasenby, M. Lattanzi, C. R. Lawrence, M. Le Jeune, F. Levrier, P. B. Lilje, M. Lilley, V. Lindholm, M. López-Caniego, Y.-Z. Ma, J. F. Macías-Pérez, G. Maggio, D. Maino, N. Mandolesi, A. Mangilli, M. Maris, P. G. Martin, E. Martínez-González, S. Matarrese, N. Mauri, J. D. McEwen, A. Melchiorri, A. Mennella, M. Migliaccio, M.-A. Miville-Deschênes, D. Molinari, A. Moneti, L. Montier, G. Morgante, A. Moss, P. Natoli, C. A. Oxborrow, L. Pagano, D. Paoletti, G. Patanchon, O. Perdereau, L. Perotto, V. Pettorino, F. Piacentini, S. Plaszczynski, L. Polastri, G. Polenta, J.-L. Puget, J. P. Rachen, B. Racine, M. Reinecke, M. Remazeilles, A. Renzi, G. Rocha, C. Rosset, M. Rossetti, G. Roudier, J. A. Rubiño-Martín, B. Ruiz-Granados, L. Salvati, M. Sandri, M. Savelainen, D. Scott, C. Sirignano, G. Sirri, J. D. Soler, L. D. Spencer, A.-S. Suur-Uski, J. A. Tauber, D. Tavagnacco, M. Tenti, L. Toffolatti, M. Tomasi, M. Tristram, T. Trombetti, J. Valiviita, F. Van Tent, P. Vielva, F. Villa, N. Vittorio, B. D. Wandelt, I. K. Wehus, A. Zacchei, and A. Zonca. Planck intermediate results. XLVIII. Disentangling Galactic dust emission and cosmic infrared background anisotropies. A\&A, 596:A109, December 2016h. doi: 10.1051/0004-6361/201629022.

Planck HFI Core Team, P. A. R. Ade, N. Aghanim, R. Ansari, M. Arnaud, M. Ashdown, J. Aumont, A. J. Banday, M. Bartelmann, J. G. Bartlett, E. Battaner, K. Benabed, A. Benoît, J.-P. Bernard, M. Bersanelli, R. Bhatia, J. J. Bock, J. R. Bond, J. Borrill, F. R. Bouchet, F. Boulanger, T. Bradshaw, E. Bréelle, M. Bucher, P. Camus, J.-F. Cardoso, A. Catalano, A. Challinor, A. Chamballu, J. Charra, M. Charra, R.-R. Chary, C. Chiang, S. Church, D. L. Clements, S. Colombi, F. Couchot, A. Coulais, C. Cressiot, B. P. Crill, M. Crook, P. de Bernardis, J. Delabrouille, J.-M. Delouis, F.-X. Désert, K. Dolag, H. Dole, O. Doré, M. Douspis,

G. Efstathiou, P. Eng, C. Filliard, O. Forni, P. Fosalba, J.-J. Fourmond, K. Ganga, M. Giard, D. Girard, Y. Giraud-Héraud, R. Gispert, K. M. Górski, S. Gratton, M. Griffin, G. Guyot, J. Haissinski, D. Harrison, G. Helou, S. Henrot-Versillé, C. Hernández-Monteagudo, S. R. Hildebrandt, R. Hills, E. Hivon, M. Hobson, W. A. Holmes, K. M. Huffenberger, A. H. Jaffe, W. C. Jones, J. Kaplan, R. Kneissl, L. Knox, G. Lagache, J.-M. Lamarre, P. Lami, A. E. Lange, A. Lasenby, A. Lavabre, C. R. Lawrence, B. Leriche, C. Leroy, Y. Longval, J. F. Macías-Pérez, T. Maciaszek, C. J. MacTavish, B. Maffei, N. Mandolesi, R. Mann, B. Mansoux, S. Masi, T. Matsumura, P. McGehee, J.-B. Melin, C. Mercier, M.-A. Miville-Deschênes, A. Moneti, L. Montier, D. Mortlock, A. Murphy, F. Nati, C. B. Netterfield, H. U. Nørgaard-Nielsen, C. North, F. Noviello, D. Novikov, S. Osborne, C. Paine, F. Pajot, G. Patanchon, T. Peacocke, T. J. Pearson, O. Perdereau, L. Perotto, F. Piacentini, M. Piat, S. Plaszczynski, E. Pointecouteau, R. Pons, N. Ponthieu, G. Prézeau, S. Prunet, J.-L. Puget, W. T. Reach, C. Renault, I. Ristorcelli, G. Rocha, C. Rosset, G. Roudier, M. Rowan-Robinson, B. Rusholme, D. Santos, G. Savini, B. M. Schaefer, P. Shellard, L. Spencer, J.-L. Starck, P. Stassi, V. Stolyarov, R. Stompor, R. Sudiwala, R. Sunyaev, J.-F. Sygnet, J. A. Tauber, C. Thum, J.-P. Torre, F. Touze, M. Tristram, F. van Leeuwen, L. Vibert, D. Vibert, L. A. Wade, B. D. Wandelt, S. D. M. White, H. Wiesemeyer, A. Woodcraft, V. Yurchenko, D. Yvon, and A. Zacchei. Planck 
early results. IV. First assessment of the High Frequency Instrument in-flight performance. A\&A, 536:A4, December 2011. doi: 10.1051/0004-6361/201116487.

PRISM Collaboration, P. Andre, C. Baccigalupi, D. Barbosa, J. Bartlett, N. Bartolo, E. Battistelli, R. Battye, G. Bendo, J.-P. Bernard, M. Bersanelli, M. Bethermin, P. Bielewicz, A. Bonaldi, F. Bouchet, F. Boulanger, J. Brand, M. Bucher, C. Burigana, Z.-Y. Cai, V. Casasola, G. Castex, A. Challinor, J. Chluba, S. Colafrancesco, F. Cuttaia, G. D'Alessandro, R. Davis, M. de Avillez, P. de Bernardis, M. de Petris, A. de Rosa, G. de Zotti, J. Delabrouille, C. Dickinson, J. M. Diego, E. Falgarone, P. Ferreira, K. Ferriere, F. Finelli, A. Fletcher, G. Fuller, S. Galli, K. Ganga, J. Garcia-Bellido, A. Ghribi, J. Gonzalez-Nuevo, K. Grainge, A. Gruppuso, A. Hall, C. Hernandez-Monteagudo, M. Jackson, A. Jaffe, R. Khatri, L. Lamagna, M. Lattanzi, P. Leahy, M. Liguori, E. Liuzzo, M. Lopez-Caniego, J. Macias-Perez, B. Maffei, D. Maino, S. Masi, A. Mangilli, M. Massardi, S. Matarrese, A. Melchiorri, J.-B. Melin, A. Mennella, A. Mignano, M.-A. Miville-Deschenes, F. Nati, P. Natoli, M. Negrello, F. Noviello, F. Paci, R. Paladino, D. Paoletti, F. Perrotta, F. Piacentini, M. Piat, L. Piccirillo, G. Pisano, G. Polenta, S. Ricciardi, M. Roman, J.-A. Rubino-Martin, M. Salatino, A. Schillaci, P. Shellard, J. Silk, R. Stompor, R. Sunyaev, A. Tartari, L. Terenzi, L. Toffolatti, M. Tomasi, T. Trombetti, M. Tucci, B. Van Tent, L. Verde, B. Wandelt, and S. Withington. PRISM (Polarized Radiation Imaging and Spectroscopy Mission): A White Paper on the Ultimate Polarimetric Spectro-Imaging of the Microwave and Far-Infrared Sky. ArXiv e-prints, June 2013.

M. Remazeilles, J. Delabrouille, and J.-F. Cardoso. Foreground component separation with generalized Internal Linear Combination. MNRAS, 418:467-476, November 2011. doi: 10.1111/j.1365-2966.2011.19497.x.

M. Remazeilles, A. J. Banday, C. Baccigalupi, S. Basak, A. Bonaldi, G. De Zotti, J. Delabrouille, C. Dickinson, H. K. Eriksen, J. Errard, R. Fernandez-Cobos, U. Fuskeland, C. Hervías-Caimapo, M. López-Caniego, E. Martinez-González, M. Roman, P. Vielva, I. Wehus, A. Achucarro, P. Ade, R. Allison, M. Ashdown, M. Ballardini, R. Banerji, N. Bartolo, J. Bartlett, D. Baumann, M. Bersanelli, M. Bonato, J. Borrill, F. Bouchet, F. Boulanger, T. Brinckmann, M. Bucher, C. Burigana, A. Buzzelli, Z.-Y. Cai, M. Calvo, C.-S. Carvalho, G. Castellano, A. Challinor, J. Chluba, S. Clesse, I. Colantoni, A. Coppolecchia, M. Crook, G. D'Alessandro, P. de Bernardis, G. de Gasperis, J.-M. Diego, E. Di Valentino, S. Feeney, S. Ferraro, F. Finelli, F. Forastieri, S. Galli, R. Genova-Santos, M. Gerbino, J. González-Nuevo, S. Grandis, J. Greenslade, S. Hagstotz, S. Hanany, W. Handley, C. Hernandez-Monteagudo, M. Hills, E. Hivon, K. Kiiveri, T. Kisner, T. Kitching, M. Kunz, H. Kurki-Suonio, L. Lamagna, A. Lasenby, M. Lattanzi, J. Lesgourgues, A. Lewis, M. Liguori, V. Lindholm, G. Luzzi, B. Maffei, C. J. A. P. Martins, S. Masi, D. McCarthy, J.-B. Melin, A. Melchiorri, D. Molinari, A. Monfardini, P. Natoli, M. Negrello, A. Notari, A. Paiella, D. Paoletti, G. Patanchon, M. Piat, G. Pisano, L. Polastri, G. Polenta, A. Pollo, V. Poulin, M. Quartin, J.-A. Rubino-Martin, L. Salvati, A. Tartari, M. Tomasi, D. Tramonte, N. Trappe, T. Trombetti, C. Tucker, J. Valiviita, R. Van de Weijgaert, B. van Tent, V. Vennin, N. Vittorio, K. Young, and for the CORE collaboration. Exploring Cosmic Origins with CORE: B-mode Component Separation. ArXiv e-prints, April 2017.

B. Revenu, A. Kim, R. Ansari, F. Couchot, J. Delabrouille, and J. Kaplan. Destriping of polarized data in a CMB mission with a circular scanning strategy. A\&AS, 142:499-509, March 2000. doi: 10.1051/aas:2000308.

A. G. Riess, L. Macri, S. Casertano, H. Lampeitl, H. C. Ferguson, A. V. Filippenko, S. W. Jha, W. Li, and R. Chornock. A 3\% Solution: Determination of the Hubble Constant with the Hubble Space Telescope and Wide Field Camera 3. ApJ, 730:119, April 2011. doi: 10.1088/0004-637X/730/2/119.

C. Rosset, V. B. Yurchenko, J. Delabrouille, J. Kaplan, Y. Giraud-Héraud, J.-M. Lamarre, and J. A. Murphy. Beam mismatch effects in cosmic microwave background polarization measurements. A\&A, 464:405-415, March 2007. doi: 10.1051/0004-6361:20042230. 
D. Scott, D. Contreras, A. Narimani, and Y.-Z. Ma. The information content of cosmic microwave background anisotropies. JCAP, 6:046, June 2016. doi: 10.1088/1475-7516/2016/06/046.

B. D. Sherwin and M. Schmittfull. Delensing the CMB with the cosmic infrared background. Phys.Rev.D, 92(4):043005, August 2015. doi: 10.1103/PhysRevD.92.043005.

G. A. Sneiderman, P. J. Shirron, R. Fujimoto, T. G. Bialas, K. R. Boyce, M. P. Chiao, M. J. DiPirro, M. E. Eckart, L. Hartz, Y. Ishisaki, R. L. Kelley, C. A. Kilbourne, C. Masters, D. McCammon, K. Mitsuda, H. Noda, F. S. Porter, A. E. Szymkowiak, Y. Takei, M. Tsujimoto, and S. Yoshida. Cryogen-free operation of the Soft X-ray Spectrometer instrument. In Society of Photo-Optical Instrumentation Engineers (SPIE) Conference Series, volume 9905 of Proc.SPIE, page $99053 \mathrm{~N}$, July 2016. doi: 10.1117/12.2232045.

A. A. Starobinsky. A new type of isotropic cosmological models without singularity. Physics Letters B, 91:99-102, March 1980. doi: 10.1016/0370-2693(80)90670-X.

J. A. Tauber, N. Mandolesi, J.-L. Puget, T. Banos, M. Bersanelli, F. R. Bouchet, R. C. Butler, J. Charra, G. Crone, J. Dodsworth, and et al. Planck pre-launch status: The Planck mission. A\&A, 520:A1, September 2010a. doi: 10.1051/0004-6361/200912983.

J. A. Tauber, H. U. Norgaard-Nielsen, P. A. R. Ade, J. Amiri Parian, T. Banos, M. Bersanelli, C. Burigana, A. Chamballu, D. de Chambure, P. R. Christensen, O. Corre, A. Cozzani, B. Crill, G. Crone, O. D'Arcangelo, R. Daddato, D. Doyle, D. Dubruel, G. Forma, R. Hills, K. Huffenberger, A. H. Jaffe, N. Jessen, P. Kletzkine, J. M. Lamarre, J. P. Leahy, Y. Longval, P. de Maagt, B. Maffei, N. Mandolesi, J. Martí-Canales, A. Martín-Polegre, P. Martin, L. Mendes, J. A. Murphy, P. Nielsen, F. Noviello, M. Paquay, T. Peacocke, N. Ponthieu, K. Pontoppidan, I. Ristorcelli, J.-B. Riti, L. Rolo, C. Rosset, M. Sandri, G. Savini, R. Sudiwala, M. Tristram, L. Valenziano, M. van der Vorst, K. van’t Klooster, F. Villa, and V. Yurchenko. Planck pre-launch status: The optical system. A\&A, 520:A2, September 2010b. doi: 10.1051/0004-6361/200912911.

The COrE Collaboration, C. Armitage-Caplan, M. Avillez, D. Barbosa, A. Banday, N. Bartolo, R. Battye, J. Bernard, P. de Bernardis, S. Basak, M. Bersanelli, P. Bielewicz, A. Bonaldi, M. Bucher, F. Bouchet, F. Boulanger, C. Burigana, P. Camus, A. Challinor, S. Chongchitnan, D. Clements, S. Colafrancesco, J. Delabrouille, M. De Petris, G. De Zotti, C. Dickinson, J. Dunkley, T. Ensslin, J. Fergusson, P. Ferreira, K. Ferriere, F. Finelli, S. Galli,

J. Garcia-Bellido, C. Gauthier, M. Haverkorn, M. Hindmarsh, A. Jaffe, M. Kunz, J. Lesgourgues, A. Liddle, M. Liguori, M. Lopez-Caniego, B. Maffei, P. Marchegiani, E. Martinez-Gonzalez, S. Masi, P. Mauskopf, S. Matarrese, A. Melchiorri, P. Mukherjee, F. Nati, P. Natoli, M. Negrello, L. Pagano, D. Paoletti, T. Peacocke, H. Peiris, L. Perroto, F. Piacentini, M. Piat, L. Piccirillo, G. Pisano, N. Ponthieu, C. Rath, S. Ricciardi, J. Rubino Martin, M. Salatino, P. Shellard, R. Stompor, L. T. J. Urrestilla, B. Van Tent, L. Verde, B. Wandelt, and S. Withington. COrE (Cosmic Origins Explorer) A White Paper. ArXiv e-prints, February 2011.

The EBEX Collaboration, A. M. Aboobaker, P. Ade, D. Araujo, F. Aubin, C. Baccigalupi, C. Bao, D. Chapman, J. Didier, M. Dobbs, C. Geach, W. Grainger, S. Hanany, K. Helson, S. Hillbrand, J. Hubmayr, A. Jaffe, B. Johnson, T. Jones, J. Klein, A. Korotkov, A. Lee, L. Levinson, M. Limon, K. MacDermid, T. Matsumura, A. D. Miller, M. Milligan, K. Raach,

B. Reichborn-Kjennerud, I. Sagiv, G. Savini, L. Spencer, C. Tucker, G. S. Tucker, B. Westbrook, K. Young, and K. Zilic. The EBEX Balloon Borne Experiment - Optics, Receiver, and Polarimetry. ArXiv e-prints, March 2017.

The Polarbear Collaboration: P. A. R. Ade, Y. Akiba, A. E. Anthony, K. Arnold, M. Atlas, D. Barron, D. Boettger, J. Borrill, S. Chapman, Y. Chinone, M. Dobbs, T. Elleflot, J. Errard, G. Fabbian, C. Feng, D. Flanigan, A. Gilbert, W. Grainger, N. W. Halverson, M. Hasegawa, K. Hattori, M. Hazumi, W. L. Holzapfel, Y. Hori, J. Howard, P. Hyland, Y. Inoue, G. C. Jaehnig, A. H. Jaffe, B. Keating, Z. Kermish, R. Keskitalo, T. Kisner, M. Le Jeune, A. T. Lee, E. M. Leitch, E. Linder, M. Lungu, F. Matsuda, T. Matsumura, X. Meng, N. J. Miller, H. Morii, S. Moyerman, M. J. Myers, M. Navaroli, H. Nishino, A. Orlando, H. Paar, J. Peloton, D. Poletti, 
E. Quealy, G. Rebeiz, C. L. Reichardt, P. L. Richards, C. Ross, I. Schanning, D. E. Schenck, B. D. Sherwin, A. Shimizu, C. Shimmin, M. Shimon, P. Siritanasak, G. Smecher, H. Spieler, N. Stebor, B. Steinbach, R. Stompor, A. Suzuki, S. Takakura, T. Tomaru, B. Wilson, A. Yadav, and O. Zahn. A Measurement of the Cosmic Microwave Background B-mode Polarization Power Spectrum at Sub-degree Scales with POLARBEAR. ApJ, 794:171, October 2014. doi: 10.1088/0004-637X/794/2/171.

M. Tristram, C. Filliard, O. Perdereau, S. Plaszczynski, R. Stompor, and F. Touze. Iterative destriping and photometric calibration for Planck-HFI, polarized, multi-detector map-making. A\&A, 534:A88, October 2011. doi: 10.1051/0004-6361/201116871.

C. L. Van Eck, M. Haverkorn, M. I. R. Alves, R. Beck, A. G. de Bruyn, T. Enßlin, J. S. Farnes, K. Ferrière, G. Heald, C. Horellou, A. Horneffer, M. Iacobelli, V. Jelić, I. Martí-Vidal, D. D. Mulcahy, W. Reich, H. J. A. Röttgering, A. M. M. Scaife, D. H. F. M. Schnitzeler, C. Sobey, and S. S. Sridhar. Faraday tomography of the local interstellar medium with LOFAR: Galactic foregrounds towards IC 342. A\&A, 597:A98, January 2017. doi: 10.1051/0004-6361/201629707.

C. G. R. Wallis, M. L. Brown, R. A. Battye, and J. Delabrouille. Optimal scan strategies for future CMB satellite experiments. MNRAS, 466:425-442, April 2017. doi: 10.1093/mnras/stw2577.

H.-Y. Wu and O. Doré. Optimizing future experiments of cosmic far-infrared background: a principal component approach. ArXiv e-prints, December 2016.

M. Zaldarriaga and U. Seljak. All-sky analysis of polarization in the microwave background. Phys.Rev.D, 55:1830-1840, February 1997. doi: 10.1103/PhysRevD.55.1830. 